University of Rhode Island

DigitalCommons@URI

Open Access Dissertations

2019

\title{
A CRITICAL EXAMINATION OF RHODE ISLAND SPEECH- LANGUAGE PATHOLOGISTS WORKING UNDER FEDERAL AND STATE EDUCATION POLICY
}

Patricia J. Murtagh

University of Rhode Island, pmurtagh@my.uri.edu

Follow this and additional works at: https://digitalcommons.uri.edu/oa_diss

\section{Recommended Citation}

Murtagh, Patricia J., "A CRITICAL EXAMINATION OF RHODE ISLAND SPEECH-LANGUAGE PATHOLOGISTS WORKING UNDER FEDERAL AND STATE EDUCATION POLICY" (2019). Open Access Dissertations. Paper 1039.

https://digitalcommons.uri.edu/oa_diss/1039

This Dissertation is brought to you for free and open access by DigitalCommons@URI. It has been accepted for inclusion in Open Access Dissertations by an authorized administrator of DigitalCommons@URI. For more information, please contact digitalcommons-group@uri.edu. 


\title{
A CRITICAL EXAMINATION OF RHODE ISLAND SPEECH-LANGUAGE PATHOLOGISTS WORKING UNDER FEDERAL AND STATE EDUCATION POLICY
}

\author{
BY \\ PATRICIA J. MURTAGH
}

\begin{abstract}
A DISSERTATION SUBMITTED IN PARTIAL FULFILLMENT OF THE REQUIREMENTS FOR THE DEGREE OF DOCTOR OF PHILOSOPHY

IN

EDUCATION
\end{abstract}

UNIVERSITY OF RHODE ISLAND

AND

RHODE ISLAND COLLEGE

2019 


\section{DOCTOR OF PHILOSOPHY DISSERTATION}

OF

PATRICIA J. MURTAGH

\section{APPROVED:}

Dissertation Committee

Major Professor:

Janet Johnson

Paul La Cava

Kathy Peno

David Byrd

RIC:

Jeannine Dingus-Eason

Dean, Feinstein School of Education

URI:

Nasser H. Zawia

Dean, The Graduate School - URI

UNIVERSITY OF RHODE ISLAND

AND

RHODE ISLAND COLLEGE

2019 


\begin{abstract}
The purpose of this dissertation was to examine the discourse of speech-language pathologists (SLPs) working in Rhode Island public schools under restrictive policy. The research questions were partly informed from a 2015 pilot study surrounding comments about a state education rule that SLPs named the nine-year rule.

In that 2015 survey, many SLPs working in RI public schools had negatively charged comments about that rule because it required SLPs to terminate speech services for students with only speech sound disorders at the age of nine. Because of the risks associated for these students, this study was value mediated. Using a critical stance, I discovered three broad themes: systems, complications and dilemmas, that shaped their identity and agency.
\end{abstract}

The systems were rooted in politics, economics, and culture stimulated by a neoliberal agenda of accountability, cost reduction, and productivity in school reform (Lipman, 2005). In the findings, the systems inadvertently deepened the complications that created ethical and professional dilemmas. An examination of SLPs' social and linguistic practices show how they positioned themselves in navigating the dilemmas. SLPs were found to navigate dilemmas three ways with: 1) what they knew; 2) what was expected; and finally, 3) what was permitted and sanctioned. At the center of their decision-making was an emotional needle that guided them. Emotions are catalytic to agency and identity (Boler, 1999), and in this study, that agency resulted in various outcomes. 


\section{ACKNOWLEDGEMENTS}

I want to acknowledge Janet Johnson, my major professor, for her guidance and support during this incredible journey. I am also grateful for my committee members' contributions: Paul La Cava, professor and researcher in special education, Kathy Peno, an adult learning expert, David Byrd, the Director of the School of Education with expertise on education policy, and Gerri August, my professor on critical discourse analysis. Also, I would like to thank Becky-Lynn Caouette who kindly agreed last minute to be an outside reader. Gerri and Janet, a special thank you for sitting down and giving me a safe space to discuss my writing challenges. My confidence and skill have grown as a result.

I want to thank my colleagues for participating in the study. They took time out of their busy schedules to share intimate stories and discuss sensitive topics. I cherish our time and work together.

My family deserves an award for tolerating the days where technology failed, and writing was not going smoothly. Their strength and encouragement carried me during this seven-year journey. When I was taking EDP 623 and 613, I was especially thankful for my sons, Sean and Patrick, who attended URI at the same time. They offered great support, love and laughs. 
Mark, my husband, thank you for your loving support and patience. As usual, he opened my eyes to the benefit of this endeavor.

I want to add a tribute to my sisters whom I shared my writing with to which they responded enthusiastically. I love you all for that support and energy. Finally, my mom who has been deceased for many years, you are constantly in my head and heart. She was a nurturer, a nurse, and a lover of education. Despite being widowed young, a mother of seven children with the youngest only 8 years-old, and battling cancer herself, she was always positive and optimistic. I will never forget when she took a philosophy class and I asked her why, she responded, "There is so much to learn and understand." Thank you for your sense of adventure and being an inspiration who modeled tremendous faith, love, and hope. 


\section{DEDICATION}

To my colleagues and students. 


\section{TABLE OF CONTENTS}

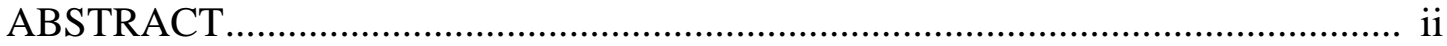

ACKNOWLEDGEMENTS .......................................................................

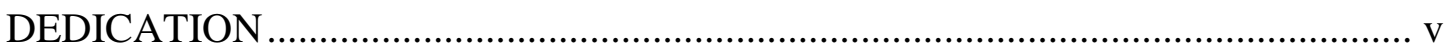

TABLE OF CONTENTS .......................................................................... vi

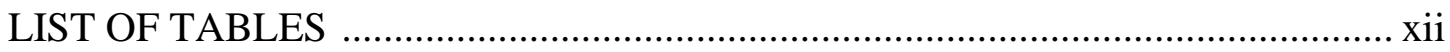

CHAPTER 1: INTRODUCTION ................................................................... 1

The SLP Professional and Mission ..........................................................4

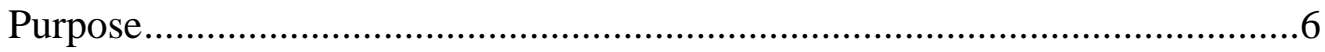

Justification and Significance ............................................................. 7

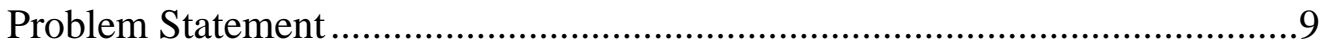

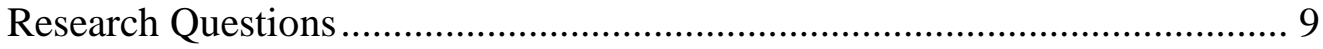

Theoretical Frameworks ........................................................................ 10

Sociocultural Model in Identity and Agency ...................................11

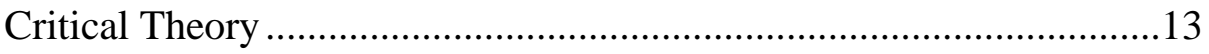

Professional Identity Formation ...................................................16

Critical Discourse Analysis and Theory …................................... 18

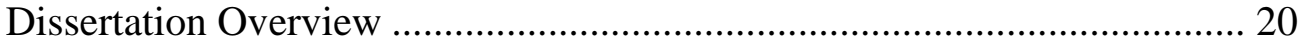

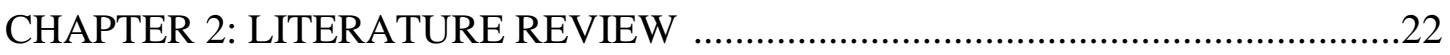

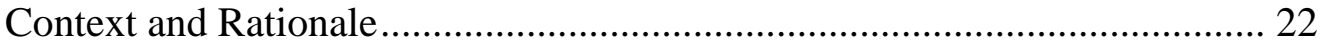

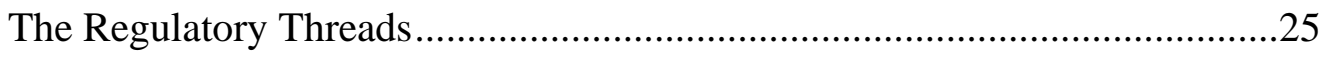

Historical Events Predating the State Rule ................................... 27

Changes in Education Landscape ........................................................28

Response to Intervention Models.............................................28

Resources Shift Educator Roles and Responsibilities .....................31 
Structures that Impact Response to Intervention ...................................37

Implications for Students and SLPs .............................................................

Students with Speech Sound Disorders .............................................42

Outcomes and Shortcomings of Authorizations ................................... 44

Relations and Perceptions Matter to SLP Work …………….........................45

Influences behind SLP Job Satisfaction............................................... 47

SLPs' Perceptions of Value in Co-taught Classes ...............................49

Professional Identity in Higher Education ..................................................49

What is Language: A Qualitative Study on SLP Role .........................50

Teacher Identity Work and Figured Worlds ..................................... 51

Social Recognition in SLP Identity..................................................52

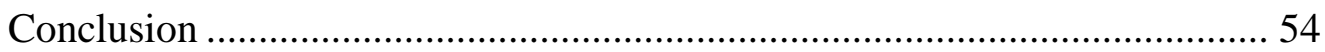

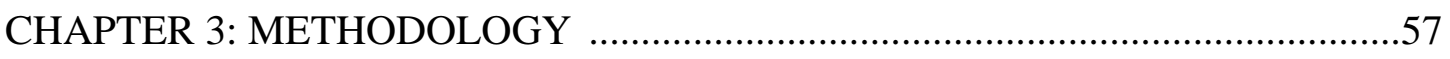

Naturalistic Paradigm Situated in My Study ……………………................57

Critical Perspective: Subjectivities and Language........................................... 57

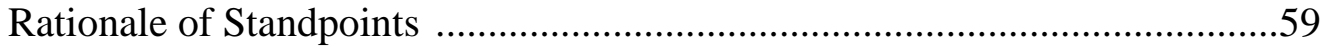

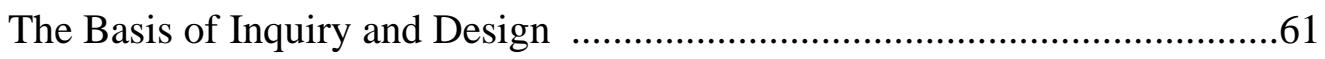

Justification for Research and Sampling ......................................................62

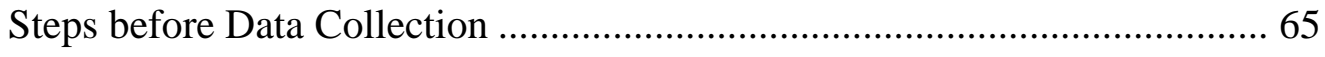

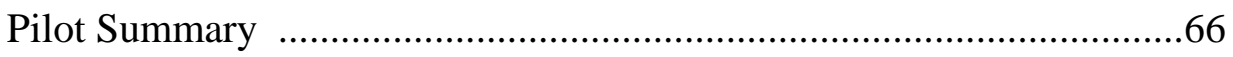

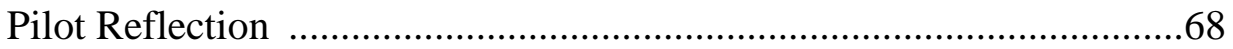




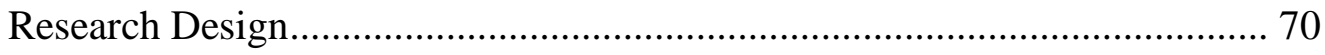

Data Collection and Sampling ............................................................

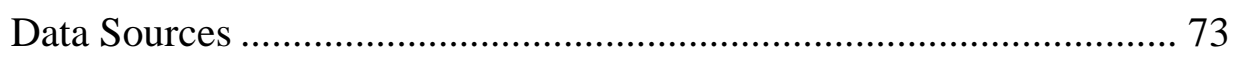

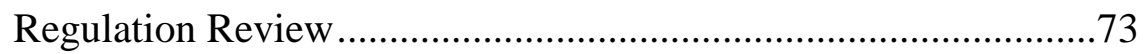

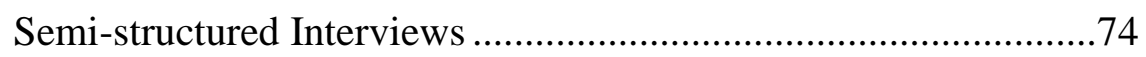

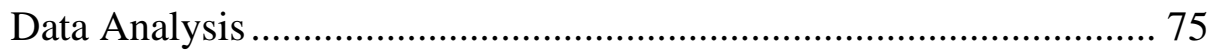

Coding and Transformation .......................................................75

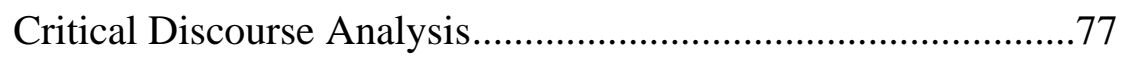

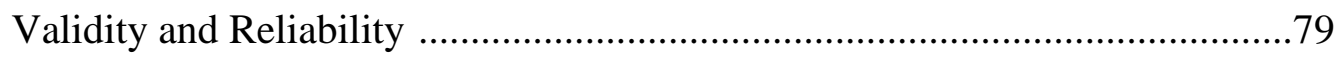

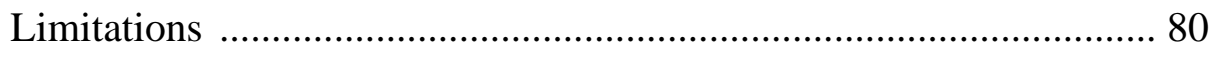

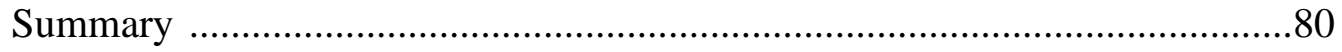

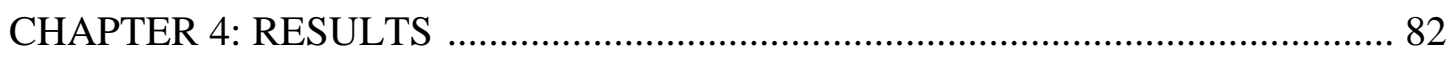

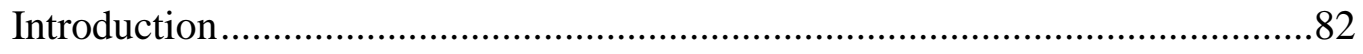

A Myriad of Factors Concerning SLP Identity and Agency...............................83

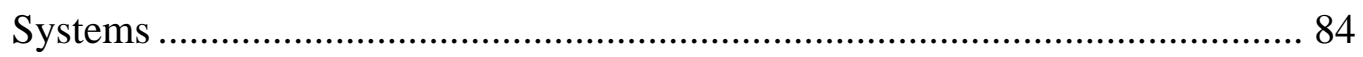

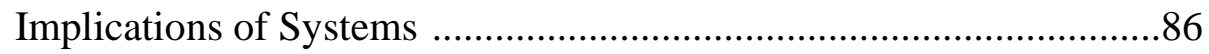

Definitions of Terms: Systems' Complications and Dilemmas.......................... 87

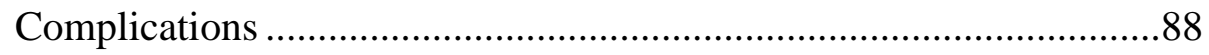

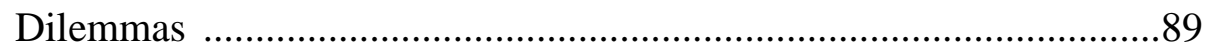

The Systems and Corresponding Complications …………….........................90

ASHA and RISHA ………………………………….................

Multiple and Conflicting Roles are Disorienting …….............91

A Mismatch between Scope of Practice and Expectations..... 99 
ASHA Thrives; RISHA Exists................................................ 101

Professional System Summary ........................................... 107

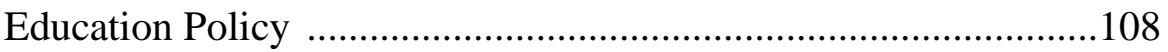

Overlapping Policy ..............................................................111

Numbers: A Driving Force in Policy …………………….....119

Policy Fails in Ensuring Success in RI ................................122

Policy System Summary ....................................................125

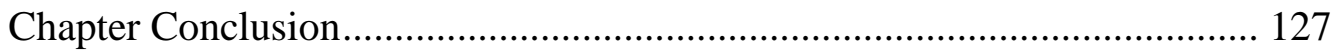

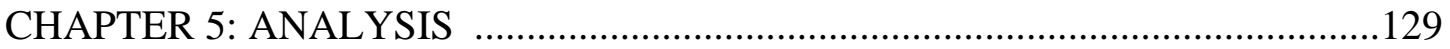

System Entanglements' Complications and Dilemmas ................................. 129

An Example of System Entanglement .............................................................131

Education Institution: People, Places, Procedures and Protocols (4P's) .......132

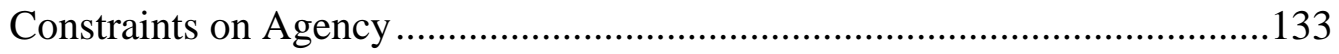

Entanglement 1: New Federal and State Regulations......................................137

An Ethical and Professional Paradox.............................................137

Sanctions, Both Legal and Economic and Threaten SLPs' Standards

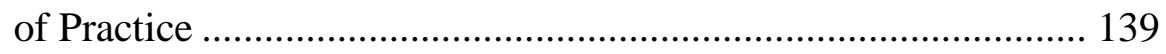

SLPs' Navigation and Agentive Processes .....................................141

Entanglement 2: ASHA's Response to Policy ...............................................143

Scope Intended to be Inclusive, Not Exclusive .............................143

ASHA's Scope not Supportive nor Consistent with Training ...... 146

SLPs Navigate Dilemmas with Humor, Idiomatic Expressions, and

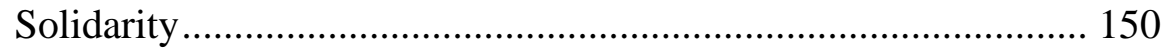


Entanglement 3: Administration Rewrites of Policy Hinder SLP Work

SLPs Sense Pushback Working with an Accountability Agenda ..153

Mismatch Among Policy and Structures Camouflage Problems....155

Education System Does Not Respond Fairly to All Students ........157

SLPs' Feelings and Values Guide Improvisations........................163

Entanglement 4: Policy Make Parent Expectations Virtually Impossible .... 165

Students' Parents Not Always Involved ...................................166

Summary of SLPs' Navigation System ................................................ 170

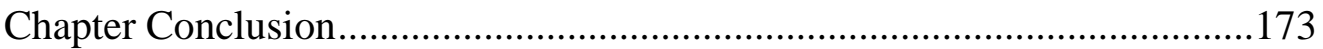

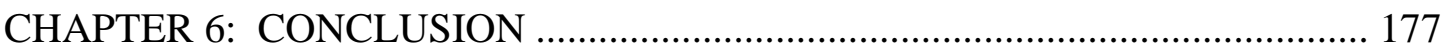

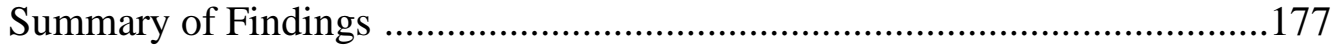

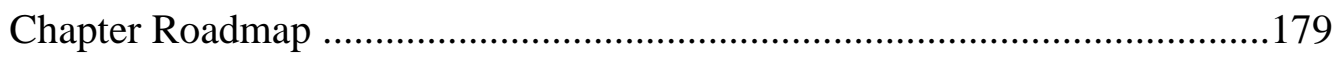

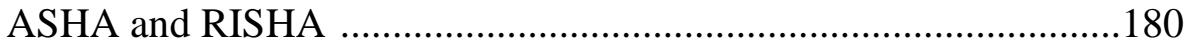

Federal and State Policy ........................................................ 180

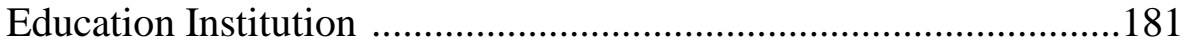

System Entanglement ........................................................... 182

What Now for SLPs, the Profession, and the Students ............................184

Practices on Behalf of SLP, Profession, and Student ...............................184

On the Right Track, Do Not Stop ..............................................184

SLPs Filling the Gap ......................................................... 185

Sensing Pushback, Somethings Up......................................... 186

School Hierarchy Matters ........................................................187

Theoretical and Research Implications .............................................. 188 
Professional Reflection and Community of Practice, a Means to

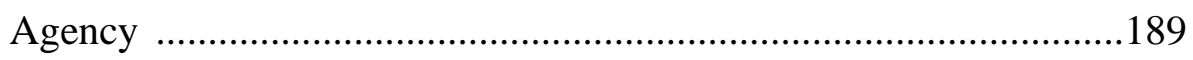

A Human Resource Frame for Managing Complications .............. 189

Future Research .........................................................................190

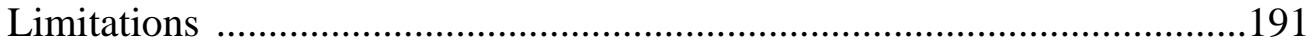

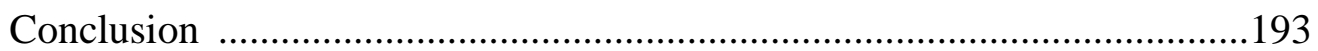

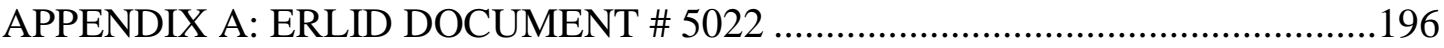

APPENDIX B: ERLID DOCUMENT \# 6746...................................................... 197

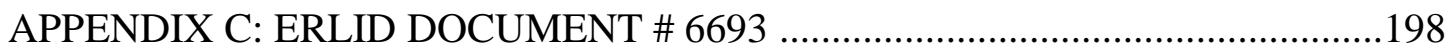

APPENDIX D: SAMPLE and WORKLOAD CHARACTERISTICS ...................199

APPENDIX E: RIC INSITUTION REVIEW BOARD (IRB) ..............................200

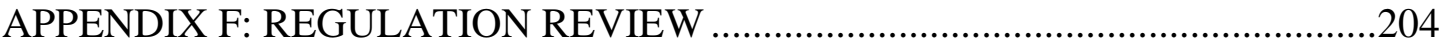

APPENDIX G: INTERVIEW QUESTIONS ................................................... 205

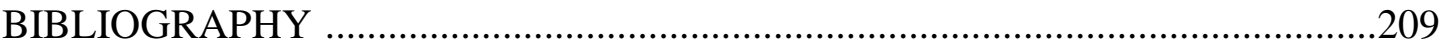




\section{LIST OF TABLES}

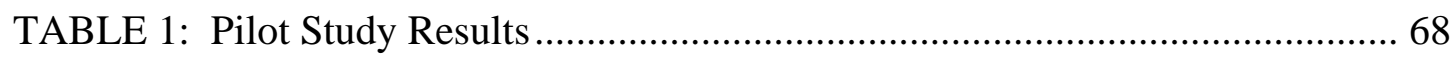

TABLE 2: Work Aspects: load, level, locale ................................................... 70

TABLE 3: Sample: Interviews and Focus Groups ............................................. 71

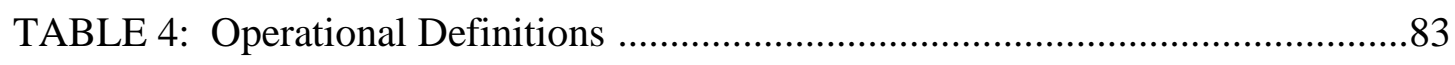

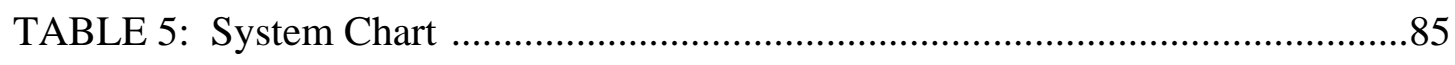

TABLE 6: Examples of Systems and Complications .............................................

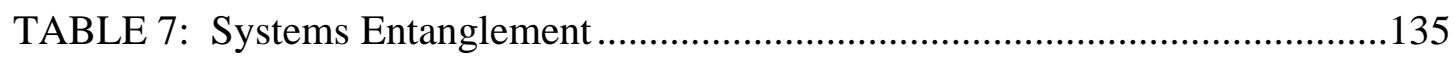




\section{CHAPTER 1: INTRODUCTION}

My longtime career as a speech-language pathologist in the Rhode Island public schools had its roots in a very different setting. Prior to working in the school system, I practiced speech therapy in a hospital. While working there, I delivered treatment plans in consultation with physical and occupational therapists. In most situations, this working partnership functioned effectively, delivering positive results for our patients.

Now I work alongside dedicated teachers yet delivering speech services to my students is a consistent challenge that does not always produce the positive results they need, and I want for them. The literature in this field demonstrates that I am not alone in facing this very serious problem. One early study found that SLPs lacked the training in curriculum-based interventions and often felt frustrated and alienated in their job (Beck \& Dennis, 1997). Another found that speech-language pathologists (SLPs) were unfamiliar with appropriate methods for delivering classroom-based and/or curriculum-based intervention services (Harn, Bradshaw, \& Olgetree, 1997). To counter my own frustration and alienation, I joined a local professional organization called the Rhode Island Speech-Language Hearing Association (RISHA) for speech-language pathologists. I found camaraderie, support, and comfort with other SLPs who were contending with similar issues. Then, in 2008, new legislation in Rhode Island triggered another chain of events that placed SLPs in the very uncomfortable position of having to terminate services to students with speech sound disorders (SSD) based on an age criterion, the 
student's ninth birthday, rather than a lack of need of these services. Among the members of the SLP community and others, this legislation became known as "the nine-year rule."

The nine-year rule limits the enrollment of students in special education after the age of nine; students with Speech-Language Impairment (SLI) and speech sound disorders (SSD) are only provided with services directed at treating this problem if students have a coexisting disability, making the SLI and SSD treatment a related service rather than a primary one (Giangreco, Prelock, \& Turnbull, 2010). When there is not a coexisting disability present, students are terminated from direct speech-language services, which removes them from the category of the need to receive special education services, thus requiring them only to be collectively monitored in the response-to-intervention (RTI) model. Monitoring and instruction are no longer delivered discretely as was the case before the application of the nine-year rule.

That 2008 legislation prompted my research into the history of the nineyear rule alongside an examination of co-existing policies which led to this dissertation. This study focuses on the dynamic interrelation and complexities of the political and cultural forces that affect education policy, explains the changes and shifts in special and general educators' roles, and finally, describes the impact on the speech-language profession in terms of adjusting their scope of practice to address the unique needs of students in Rhode Island public schools. 
In addition to a traditional literature review, this inquiry included interviews of SLPs across the state of Rhode Island working at elementary and secondary level schools. Using a demographic survey, I was able to assemble two focus groups: 1) a diverse group of working SLPs from across the state; and 2) a group of middle school based SLPs. The narratives of the group members' experiences were highly informative for a number of reasons. Some of the SLPs had been working with elementary students before the introduction of the nine-year rule and after; some had been employed only after the onset of the rule; and some worked with secondary students who had been impacted by that rule as a nine-year old. Although many of the narratives indicated a felt sense of a lack of agency in the SLPs' day-to-day work, the focus groups themselves were agentive in that they gave SLPs a safe place to voice their beliefs and experiences. Additionally, the follow-up face-to-face interviews provided an opportunity for these SLPs to engage at a more personal level and delve into issues that had surfaced in the focus groups. As a result of the findings, this study exposed systemic issues that shaped SLPs' decisionmaking, agency, and professional identities.

In this chapter, I provide an overview of the study's purpose and a problem statement based on a state rule's passing and the significance of this study for a subgroup of students impacted and the professionals serving them. I describe a political scenario that currently exists in today's education reform. I discuss the SLP professional and practices in terms of identity and agency and 
the potential contributions they can make for their students and their

profession. In addition to the identity and agency socio-cultural model, I explain other theoretical frames that help to understand the problem and answer research questions.

\section{SLP Professional and Mission}

Two professional organizations, the American Speech-Language and Hearing Association (ASHA) and Rhode Island Speech-Language and Hearing Association (RISHA), expect the SLP professional to uphold fair and responsible principles (ASHA, 2009; RISHA, 2009). Yet, the day-to-day results of their obligation to enforce a state rule called the nine-year rule were often in conflict with those principles. A 2015 survey of 340 Rhode Island public school SLPs indicated that in practice, they had found the rule to be "unfair and discriminatory" (Robinson, 2015). They elaborated that the rule negatively impacted their students' academic progress and their ability to perform their work in a manner that reflected their training and principles of the profession.

In their attempts to reconcile their professional principles, Rhode Island SLPs encountered roadblocks. Moreover, in the case of veteran SLPs, their years of knowledge and experience made satisfying these principles even more of a dilemma, since they had not been previously required to compromise those principles. Now, in some instances, their attempts to find ways to deliver appropriate services did indeed compromise their personal and professional practices. These actions led them to question their professional identity in the 
workplace, both in how they saw themselves in their role as an SLP as well as how their other colleagues saw their role within the greater school setting. Eraut (2008) defines professional identity formation as the integration of a professional's background and values combined with the norms of the profession. Through a lens of professional identity, this study offers insights into how policy impacts the professional practices of SLPs.

Rhode Island Speech-Language and Hearing Association's parent organization, the American Speech-Language and Hearing Association (ASHA), credentials SLPs as competent practitioners who pledge "excellence in practices and advocacy of those we serve" (Dublinske, 2015, p. 2). Since speech-language impairments are the second largest disability category (Scull \& Winkler, 2011) and language disorders are the hallmark of learning disabilities (Cabbage, 2015; Katts \& Cami, 1986; Apel \& Henbest, 2015), SLP advocacy on matters concerning students is imperative. The American Speech-Language and Hearing Association's opposition to the nine-year rule was also supported by Alexa Posny, the former director of the Office of Special Education Programs (OSEP) at the U.S. Department of Education (Al-Mondhiry, 2008). Of note is that in spite of these professionals' opposition to the rule, at the time of the writing of this study, the nine-year rule, which was instated in 2007, has not been changed and SLPs continue to operate under political and cultural ambiguities that make their work frustrating. 


\section{Purpose}

The purpose of this study was to examine the processes shaping the identities and agency of SLPs working with federal and state policies that limit their ability to provide their students with the services they need. In the process of finding ways to countermand this dilemma, SLPs created situations that conflicted with their professional and personal principles. According to Clarke, teachers have an ethical obligation to reflect on identities and engage in identity work (2009). Clarke adds that when the integrity of teachers' work is threatened, they have opportunities to engage in agency and identity work. SLPs are agentive beings who utilize social and linguistic resources that help them resist identities that position them in undesirable ways (2009). For example, the enactment of the nine-year rule along with the Rhode Island education system's adherence to the Response to Intervention (RTI) principles, which were introduced in the regulatory notes of the 2004 reauthorization of the federal Individuals with Disabilities Act (IDEA 2004), made it difficult for SLPs to follow the progress (or lack thereof) of students with a history of speech learning disabilities.

As a result of these administrative changes, whenever possible SLPs have had to find new and sometimes taxing ways to address learning issues that they know their clients have, whether the regulations acknowledge them or not. They organize after-school clubs, assist families with referrals to outside consultants, and provide consultation and/or co-instruction with content teachers. At other times, SLPs determine eligibility for special services by either taking on students prematurely or creating a case for another disability. All of this must fit in with 
their other duties and adhere to a code of ethics that their job covers. It is the combination of these aforementioned issues that creates dilemmas that compromise their work. This study set out to examine how SLPs' agency was constrained, and professional identities shaped.

\section{Justification and Significance}

Rhode Island is the only U.S. state with a regulation (the nine-year rule) that limits speech-language services such as articulation, fluency, and social pragmatic therapies (Giangreco et. al., 2010). Subsequently, this approved regulation deprives a specific subset of Rhode Island students of the right to receive speech services. A review of this regulation reveals a series of amendments and formal proceedings that included postings of hearings and an invitation for public comments. The process was chaotic marked by clerical errors and the state's inattention to inform the public of a scheduled hearing. That mishap deprived the public's access to the hearing and limited their ability to adequately comment on the hearing and the implications this rule would have for certain students.

In 2010, the nine-year criterion was embedded in special education eligibility requirements and officially authorized. Policy is often driven by an agenda of controlling cost through accountability and productivity measures, all of which does not necessarily address students' unique needs (Lipman, 2005). I suggest that this rule was perhaps caught up in that agenda and I offer the following examples to illustrate how that agenda functions. 
Special education is costly with pupil per school expenditures (PPEs) exceeding general education costs by $40 \%$; from the period of 1996 to $2005,40 \%$ of education spending went to special education (Scull \& Winkler, 2011). National trends showed that the number of public-school students with disabilities peaked during the 2004-2005 school year (Scull \& Winkler, 2011). Of note, speech-language disabilities are the second highest disability category (Scull \& Winkler, 2011; Giangreco, Prelock, \& Turnbull, 2010). Percentages vary from state to state and district to district. During that year, Rhode Island had one of the highest percentages of students receiving special education, at 18\%; that state average has since decreased to 12\% (Scull \& Winkler, 2011; rikidscounts, 2017). In response to those high numbers, revisions to IDEA (2004) resulted in the introduction of RTI and other changes in special education disability criteria.

Policies, such as RTI, function ambiguously and chaotically when they serve multiple agendas of accountability, productivity, and cost reduction. To complicate matters, Rhode Island SLPs are contending with the state authorization of the nine-year rule. These reform trends are consistent with a neoliberal agenda in which productivity considerations and accountability measures appear to be the means to solving cost and budget issues rather than meeting students' needs (Lipman, 2005). Consequently, SLPs' professional identity is constrained when they struggle to adhere to a mission of excellence in practice and advocacy for their students.

Identity is defined as the roles learned, developed, and assigned value within cultural models and the discursive practices are the medium that sustain, 
resist, or recruit those identities (Holland, Lachicotte, Skinner, \& Cain, 1998;

Gee, 2014). Therefore, this study intends to examine and better understand SLPs' identity processes as they operate under restrictive policy while adhering to ASHA's mission within their school settings. Although ASHA has conducted surveys that rate and reveal barriers to SLPs' job satisfaction, SLPs' access of social and linguistic resources in these situations has not been examined (ASHA, 2000, 2008, 2010, 2011, 2014, 2015).

\section{Problem Statement}

Existing literature indicates that the factor of teacher identity is commonly examined; however, that is not the case for SLPs working in public schools (Hatt, 2007; Clarke, 2009; Robinson, 2007). Although satisfaction, collaboration, and training factor into identity and agency (Beck \& Dennis, 1997; Elkinson \& Capilouto, 1994; Fallon \& Katz, 2011), these studies on SLPs working in public schools do little to explain the processes of identity shaping. Moreover, the unique situation that exists specifically in Rhode Island warrants a thorough and thoughtful investigation of SLPs' discursive and social practices.

\section{Research Questions}

Through a critically based and naturalistic qualitative methodology, this study explored how SLPs find meaning in how they are culturally and historically situated by seeking answers to the following questions.

(1) What challenges and tensions do SLPs experience in delivering speech services to students targeted by the nine-year rule in relation to RTI, and what are some of the ways in which they respond? 
(1a) What do contextual factors such as school district, grade level, and RISHA membership contribute to SLPS' responses to the nine-year rule?

(2) What sorts of experiences and/or values do SLPs perceive as significant in the formation of their professional identities?

(2a) How do SLPs' relationships with one another, their perceptions of themselves, and their understanding of how they are perceived by other colleague's influence their professional identities and practices?

(3) How do SLPs integrate their values, ethics, professional experience, and/or background/education with the norms of the profession and how do their preferred practices align with ASHA's professional standards?

\section{Theoretical Frameworks}

This study operates through the convergence of four theories: (1) identity and agency in cultural and social worlds (Carspecken, 1996; Holland, Lachicotte, Skinner, \& Cain, 1998; Gee, 2011); (2) critical theory (CT), including the transformative adult learning theory (Gee, 2011; Boler, 1999; Foucault,1979; Carspecken, 1996; Brookfield, 1993; Mezirow, 1985); (3) professional identity formation (PIF) (Eraut; 2008 Branch, \& Frankel, 2016; Gee, 2014; Clarke, 2009; and (4) critical discourse analysis (CDA) (Gee; 2001; Foucault, 1979). These theoretical frameworks helped to explore the underpinnings of identity and agency. They explain how socialization, context, relationships, and normative structures factor into one's agency and identity. These frameworks intervene on behalf of marginalized groups, as such they are transformative, value mediated, and justice oriented.

In summary, while CT and CDA are value-mediated and justice-oriented, PIF and adult transformative learning incorporate reflexivity. All of these theories help SLPs account for subjectivities that have ethical implications in professional practice. 


\section{Sociocultural Model in Identity and Agency}

The first theory that frames this study is an explanation of the workings of sociocultural influence on shaping agency and identity (Eraut, 2008; Holland et al., 2002; Gee, 2011). Holland et al. (2002, p. 49) conceives of a socioculturally constructed arena, referred to as a "figured world." In this world, behaviors are assigned values and "particular outcomes are valued over others" (Holland et al., 2002, p. 52). Figured worlds come into being through artifacts and practices. Johnson (as cited in Carspecken, 1996) adds that cultural artifacts and practices yield different meanings depending on how they are interpreted and valued. Likewise, Gee (2014) references figured worlds in which participants locate their identities based on experiences, perceptions, and values.

In response to those influences, Gee (2011) describes how individuals create "identity kits" that function as tools for learning and understanding, and in that sense, participants dress and rehearse for scripted roles like actors (as cited in Richmond \& Kurth, 1999, p. 678). Of note, adult learning studies claim Gee's identity kits help shape identity formation (Gee, 1991;1990; Lave \& Wenger, 1991; Richmond \& Kurth, 1999). Gee (2011) and Holland et al. (2002) agree that social recognizability and the values assigned to behaviors are also important to identity. However, in that process, perceptions of others and selves are equally important. For instance, in this study, SLPs were conflicted when they thought one way, yet chose to act by how they were valued and/or wanted to be recognized (Holland et al., 2002; Boler, 1999). For example, a novice SLP 
abandons her lesson when a teacher's lesson was valued more important because of grading and accountability standards. In these moments, SLPs encountered dilemmas, and regardless of the scripts, experienced discomfort.

Relying on socialization and/or cultural models makes decisions simpler (Branch \& Frankel, 2016). However, in the following example, one SLP's morally based decision was complicated due to existing policy. When deciding whether to extend services for a student with a speech-language impairment on his/her ninth birthday, a Rhode Island SLP might deviate from the rule. By acting autonomously, the SLP contradicts the principles of a federal mandate, which is premised on the appropriate utilization of interventions based on a universal monitoring system. For instance, an after-school speech session, which is not part of that system, would likely result in student growth; however, that outcome would not be valued since only outcomes associated with identified response interventions are taken into account.

Conceptions of identity. Identity is conceived of more creatively when habituation is challenged by combining what we know with what we can imagine (Holland et al., 1998). That mix of imagination and knowledge moves us in and out of awareness, so that one is more likely to envision rather than assume and follow. Although linguistic choices and cultural artifacts often function as positional markers that cut across figured worlds (Ullman, 2012), imagination also moves identities and the figured worlds we inhabit (Holland et al., 1998). In this study, SLPs improvised remedies for restrictive systems and enacted changes 
with the intention of helping; however, those changes often brought on potential problems. Most education research focuses on helping and creating solutions; however, critical theory argues that such acts unintentionally perpetuate the status quo when the complexities prompting those solutions are under-examined (Creswell, 2014, p. 205).

The impact of identity in the school setting. Understanding both the process of how one defines one's identity and the impact identity has within any social setting is an important analytic tool for understanding the operations of schools and the society that works within their walls (Nygreen, 2013). In these social institutions, one's acts are contextually bound when the responses to those acts vary based on when, where, and with whom they are situated (Eraut, 2008; Brookfield, 1993; Taylor, as cited in Merriam, Caffarella, Baumgartner, 2007). Because of this, ambiguities and subjectivities are not uncommon. However, it is the individual who contributes to the work of identity formation (Blackburn, 2003). In this study, institutionally-bound SLPs' agentive capabilities and identities were explained by their location and relationships. For example, one SLP's choice to change her way of documentation that served to protect her rather than informed the trajectory of a student's intervention was the result of hearings and parental concerns that SLPs were not servicing students sufficiently.

\section{Critical Theory (CT)}

The second frame of this study was critical theory, which is value-oriented and concerned with social inequalities. Since the nineteenth century, education 
struggles were caught up in issues surrounding social structure, power, culture, and human agency. Critical research aims to refine rather than describe those issues (Carspecken, 1996, p. 254). Discourse and school institutions are sites of control in that participants are ranked and ordered, and positions matter; however, through participation, inhabitants can reconceptualize identity (Nygreen, 2013, p. 9; Holland et. al., 1990; Gee, 2011). Within those sites of control, SLPs' testimonials and emotions factor into disorienting dilemmas, both of which have transformative potential (Boler, 1999; Mezirow, 1985). Adult learning theorists have also applied aspects of critical theory in their research (Brookfield, 1993). The following paragraphs contain a discussion of Boler's contributions to critical theory as well as the transformative aspects in adult learning theory.

Emotion: critical and catalytic to agency and identity. In terms of empowerment, Boler (1999) conceives of a pedagogy of discomfort to counterbalance social control. Boler argues that testimony and bearing witness are effective ways to disrupt harmful power structures and supports Dewey's sentimental listening as a way to disrupt habits. In this study, SLPs' discourse was emotive as it mediated economic constraints. As cited in Boler (2005, p. 3), Judith Butler (1977) states social and political culture predetermines certain voices and articulations as unrecognizable, illegitimate, and unspeakable. However, in this study, SLPs were emotionally situated and supported each other's narrative accounts as legitimate and recognizable. SLPs' feelings, though often disguised with metaphors, were nonetheless present in many situations and were crucial to counteracting their experiences of dominance and power. 
Critical theory aspects in adult learning theory. A number of adult learning theorists have brought critical theory (CT) to the study of adult education. For example, Welton (1995) believes that certain aspects of critical theory inform adult learning theory and practice. In this study, the SLPs were faced with an interplay of systems that they could not actively control. Welton (1995) states that CT helps people stop responding to situations as passive victims, colluding in their own domination by external forces (p. 250). Brookfield (2000) also proposes applying a critical theory framework so that adults learn to recognize the predominance of ideology in their responsiveness within the institutions they inhabit (p. 257). Brookfield's work (as cited in Merriam et. al, 2007, p. 146) on adult learning reflects assessments that are based on analyzing power relations and hegemonic assumptions. An example of a hegemonic assumption in adult learning theory is the idea that "adult education can guilt staff into taking on more work and reducing costs" (Merriam et al., 2007, p. 257). According to Brookfield (1993), critical theory's strength lies in its capacity to critique inhabited structures whereby one moves beyond mindless decisions and does not assimilate them (p. 255).

Speech language pathologists inhabit systems and structures that require interaction with educators, parents, and administrators. External factors, i.e., policy and work experience, and internal factors, i.e., emotions and sense of value, factor into the quality and quantity of their responsiveness. In terms of constraining SLP agency and shaping their identities, the third framework of this study, professional identity formation (PIF) acknowledges experiential factors 
while critical theory in adult learning emphasizes the influences of systemic forces and hegemonic assumptions.

\section{Professional Identity Formation (PIF)}

Professional identity formation (PIF) contains aspects of both adult learning and critical theory. Professional identity formation is a commonly applied research tool in the medical field and has only recently been introduced into teacher training programs (Branch \& Fraenkel, 2016; Eraut, 2008; Johnson, Wilson, Cowin, \& Young, 2012). The development of professional identity develops from the integration of background, values, and experiences combined with the social norms of a profession (Johnson et al., 2012). Paterson, Higgs, Wilcox, \& Villenue (2002, p. 433) define professional identity as "a sense of being professional." To ensure professional development in teaching occurs, Reid (2008) argues that universities apply pedagogies and develop curricula that prepare graduates for the world of work. For instance, self-directed learning and critical evaluation permit personal adequacy and satisfaction in one's performance of an" expected role" (Paterson et al., 2002, p. 433). As such, pedagogies and curricula should include these personal aspects in shaping one's professional identity.

According to Paterson et al (2002) adequacy and satisfaction are gained as an individual develops values and behaviors when meeting expectations of a professional membership. ASHA maintains a code of ethics that defines what the SLP profession values and expects of its practitioners as far as duties, responsibilities, and fairness (ASHA, 2016, p. 1). When SLPs try to adhere to a 
mission of excellent practices and advocacy for students, they encounter professional and ethical dilemmas. Examples of ethical violations include malpractice, student neglect, and/or billing and record fraud. Consequences of violations can be detrimental, i.e., revocation of one's license and/or membership, suspension and the withholding of practice. Others are less damaging to one's career, i.e., private or public reprimand (ASHA, 2016). Clouder (2005, p.508) describes dilemmas as 'messy realties in the world of work' and challenges academics to rethink how they prepare preservice teachers, or in this case SLPs. Planning for messy realities include understanding influences into one's sense of being and feelings of adequacy and satisfaction; all of which shape professional identity.

The role of reflexivity in professional identity formation. The theory of subjectivities, which is the authoring of oneself, explains how identity complicates and/or impedes a teacher's account of her/himself (Clarke, 2009). Clarke offers a vignette in which three paradoxes surrounding excess and difference contain an ethical scope and guide a teacher in her/his ongoing social processes of identity construction. The identities are constantly patched with predisposed discourses that often position them undesirably; reflexive discourse had a mediating effect in which another identity was possible. For instance, a teacher struggles with discipline because by nature s/he does not scold; however, s/he negotiates that tension with reflection and creates a positive learning environment that is student-centered. Drawing on Foucault's notion (as cited in Clarke, 2009) of self-narration and reflexivity, SLPs can renegotiate an identity 
that offers other opportunities and possibilities. Briggs (2007) adds an integration of personal knowledge with external ways of knowing also factors into identity shaping. In this study, SLPs negotiated tensions when they decided to meet with students informally, make them eligible under another disability, or identify student eligibility at a younger age.

\section{Critical Discourse Analysis (CDA)}

Gee (2014) argues that any theory of discourse analysis is made up of a set of tools with which to analyze language in use. He claims this form of analysis can cover theory that is tied to language structure surrounding social, political, and cultural terms (Gee, 2014, p. 1). This study followed his rationale for the use of CDA by combining it with the following explanation of studies on relationships between language and power.

Language, whether it is transmitted orally, by writing, or even through a physical gesture, as in body language, is the main way that humans communicate with each other. Gee (2014) conceives of language as a tool for making sense of ourselves in our culture. Foucault (as cited in Ullman, 2012) believes that discourse is knowledge that is culturally produced and historically situated. Based on these tenets, language is central to how knowledge is accumulated, formed, and appropriated. Therefore, when individuals talk and act a certain way, they influence the definition of situationally applicable values and norms; for some theorists, this suggests different kinds of social regularities. Carby (as cited in Rothenberg, 2005) argues that everyone in society is shaped in some political manner and in order to make visible what is rendered invisible, the normative 
state must be questioned (p. 11). However, when a social group rationalizes actions that do not match its more highly valued past actions because they believe that they now have no choice but to conform to a new normative state, these older and more valued positions on social regularities seem to disappear.

In this study, the social and discursive practices of SLPs were critically examined using CDA language tools to reveal their positionality, opposition to policy and work constraints, and the process by which they navigated professional dilemmas of a moral nature. Also, SLPs' humor paired with intertextual devices, i.e., metaphors and analogies, were socially recognizable, and because of that, carried out two functions. First, the SLPs achieved consensus and validation recruiting others to their beliefs; and second, they experienced comic relief surrounding their frustrations and sometimes less than honorable responses to power. For instance, one SLP described her work as over and beyond; however, a hearing that questioned her practices, and not the policy that resulted in a decrease of speech services, had another outcome. When she compared her situation to being thrown to the wolves to which another elaborated, or jailed, she chose to not practice over and beyond. Although the SLPs supported her, the roots of the injustice, being policy and administration, remained unnoticed and students with speech needs were inadvertently impacted.

This study's theoretical frameworks of cultural models in identity and agency; critical theory; adult transformative learning theory; and professional identity formation, offered critical analysis and alternative conceptions of identity and agency for the Rhode Island SLPs working under a restrictive education 
policy. Guided by a theoretical understanding of the linguistic and social mechanisms, a methodological approach using critical discourse analysis identified the mechanisms of power and belief systems that were being taken for granted.

\section{Dissertation Overview}

Chapter I introduced the dissertation beginning with the purpose of the study, a description of its participants, and the context in which they were situated. The justification for this study centered on political and educational histories consistent with a neoliberal reform agenda. Finally, the study outlined the theoretical frames that guided this inquiry.

Chapter 2 reviews political histories, the shortcomings of overlapping policy, the implications for specific students and the SLP profession, and significance of perception and value in teacher agency and identity through a review of the literature. Much of the subject matter of the reviews encompassed the implications of SLPs' work on behalf of students with speech sound disorders and its relation to literacy.

Chapter 3 outlines this study's methodology, which was mainly coding processes alongside critical discourse analysis (CDA); and an overview of the study's research design. Chapter 1 introduced CDA in terms of its relation to social, political, and cultural terms. In this chapter, the methodological side of CDA helps to sort out the details of those terms.

Chapters 4 and 5 present the findings of this study. Chapter 4 mainly answers research questions \#1 and \#2 which found SLPs improvised less 
orthodox responses to complications stemming from two systems: policy and professional organizations. Chapter 5 mainly answers research question \# 3 which examined the relationship of systems within the education institution, namely system entanglement, in which SLPs were emotionally situated. That ultimately mattered to their navigation of systemic dilemmas and their agency and identities. Chapter 6 provides the implications of this study's findings as well as recommendations for future research and practice. While individual speechlanguage pathologists may gain comfort in the fact that they are not alone in their dilemmas, this study identifies several changes that would better prepare SLPs in their day-to-day work experiences, one of which includes a better curriculum design in universities. 


\section{CHAPTER 2: LITERATURE REVIEW}

The purpose of this literature review is to situate speech-language pathologists (SLP) who work in Rhode Island public schools within a framework of the wider context of education policy reform and any resulting professional restrictiveness. In doing so, this literature review focuses on policy and SLP practices in four main areas, all of which result in significant outcomes for the SLPs and their students: (1) regulatory threads; (2) changes in the education landscape; (3) implications of those changes on students and SLPs; and (4) relational links between policy and participants' agency. An investigation into these areas facilitated this study's inquiry into the factors shaping SLPs' identity and agency while working in Rhode Island Public Schools.

\section{Context and Rationale}

I take a critical perspective in order to reveal inequities and power structures, explain the manner by which power is mediated, and describe implications for the SLP profession and the students it serves. One of the tenets of critical theory requires that histories be revisited. This chapter details federal and state education reform initiatives and studies related to neoliberal reforms and the corresponding implications alongside the cultural and professional shifts in education (Scull \& Winkler, 2010, Fuchs et al., 2010). According to Machi and McEvoy (2012), a literature review should summarize existing knowledge and produce a position on that knowledge. To accomplish this, I provided context and background on the topic, laid out by a defense that identified, explored, 
advocated, and then revealed what was not known before (Machi \& McEvoy, 2012, p. 2). I laid out influences in the SLP practice; explored the prevalent regulatory processes and their shortcomings; and advocated on behalf of those impacted, including SLPs and students. The review concludes with the addition of critical studies conducted on related professions and a proposal that similar research be applied to the public-school-based SLP.

Prior to this research, I was unaware of how markedly the dynamic and complex nature of the intersection of politics, economics, and culture, as opposed to conflating them into a single presence, could affect the work of members of the SLP profession as well as general education teachers. The literature demonstrates the presence of a neoliberal agenda with threads of accountability, productivity, and cost reduction woven throughout (Lipman, 2005). For example, an accountability measure required that teachers pass a test on their knowledge of phonetics (Paterson, 1998). Yet according to Paterson (as cited in Allington, 2002), studies on the relationship between that policy and practice are lacking. This is important to note when students' needs vary, and teachers teach differently.

State funding correlates with school capabilities, resources, and teacher effectiveness (Allington, 2002). With the advent of Response to Intervention (RTI), Title 1 funds shifted from special education to general education (Kaloi, 2011). Hence, the blurring of special and general education spending rendered any such state-level analysis complicated and less useful (Scull \& Winkler, 2010; Allington, 2002). To complicate matters, Title 1 funding have allowed district 
superintendents to obtain funding while only minimally meeting federal guidelines (Allington, 2002).

As seen above, Title I funding has led to situations that have not fulfilled the federal government's initial intention to improve the quality of education for all students (Allington, 2002; Puma, Karweit, Price, Ricciuti, Thompson, \& Vadid-Kiernan, 1997). Allington (2002) calls these situations "minimum compliance models" (p. 241). According to Johnson (as cited in Allington, 2002), superintendents and other school administration officials consider their responses to be in compliance with the federal guidelines insofar as being accountable to its structures; yet the end result of how the funding is used differs in varying degrees, school by school. Thus, acting autonomously without actually having real autonomy has turned out to be a situation of basically "no accountability" (Allington, 2002, p. 242) and therefore, offering no real reason to look more deeply into the results. Another way to explain this is that when someone is told how to do his or her job, it is less likely that the person will feel responsible for the outcome should that initiative fail to work. Another complicating scenario is that education programs are not adequately monitored for success.

In response to this problem, Allington (2002) suggests following a selfassessment guideline rather than assuming the "accountability without autonomy" stance. Similar to what Allington (2002) advocates, my research is an assessment of education policy in order to ascertain the seeds of neoliberalism, the roots of the resulting injustices, and finally the growth and perpetuation of the resulting 
complications in the education system as it applies to the student clients of Rhode Island's public school speech-language pathologists.

\section{The Regulatory Threads}

Accountability without autonomy may have been the case in Rhode Island when SLPs eventually implemented a questionable state-mandated rule, after having voiced opposition and been unacknowledged. Therefore, it is important to investigate how this so-called "nine-year rule" came to exist and why this rule, which SLPs continue to enforce, is problematic for students who quite often are being prematurely discharged from speech services at age nine.

A thread of regulatory revisions concerning the instigation of this rule began in December 2007, although the actual rule was not officially adopted under its formal title of Special Needs Regulation until July 3, 2010. These revisions were arbitrarily explained in files catalogued by "ERLID \#s," the Rhode Island acronym used for all state department regulations catalogued by "ERLID \#s," the Rhode Island acronym used for all state department regulations and are currently filed in the Office of the Rhode Island Secretary of State and accessible through its website. At some point during the negotiations, the Board of Regulations had to file a repeal due to a technical revision for a "clerical error," so the regulation was amended and refiled (see Appendix B for ERLID \# 6746 explanation) ; hence, the regulations, in fact, were not authorized until July 3, 2010 (https://rules.sos.ri.gov/regulations). Therefore, by September 2008, the Rhode Island public school SLPs were enforcing a new rule that was not yet 
fully understood or supported by them and at that point had been only cursorily authorized ([RIDE Reg. $§ 300.34$ (15) and 300.39 ((2) (i); ASHA/RISHA survey]). Included in this final revision of the Special Needs Regulation was the so-called nine-year rule, which stipulates that speech services for students with "speech only" services be terminated from those services on their ninth birthday. Only those students who are nine and older and present with additional/other disabilities can be seen by SLPs for speech therapy because now, that therapy comes under the classification of an approved "related service." Consequently, this approved regulation deprives a substantial subset of Rhode Island students of speech services. It is important to note here that Rhode Island is the only state with such a stipulation.

Although the state's SLPs and the parents of students impacted by the rule attended Board of Regents public hearings, their comments were not acknowledged and little public debate ensued (G. Robinson, personal communication, March 11, 2015). Moreover, the time period between the posting of the public hearing dates and the scheduled hearings was less than three weeks (see Appendix A for dates on ERLID \#5022), a relatively short time period for notices of this nature. This accelerated timeline corroborates McGuinn's (2015, p. 17) findings that "the speed and process by which states have adopted [regulations]... and the way policy is pushed through happens largely without much public discussion or debate." On March 8, 2007, an official statement from the U.S. Department of Education criticized this rule. ASHA's strong opposition 
to this rule was supported by Alexa Posny, the former director of the Office of Special Education Programs at the U.S. Department of Special Education, when she stated in a letter that "a speech and language impairment [that] affects the child's educational performance must be determined on a case-by-case basis, depending on the needs of a particular child and not based only on age or grade performance ..." (Al-Monhiry, 2008, p. 7). As of this date, there has been no change or response from Rhode Island's Department of Education vis a vis the 2010 regulation.

\section{Historical Events Predating the State Rule}

According to Carspecken (1996), not addressing the structural resources of differential power is not addressing the cultural and social processes. The historical and social implications of centralized and decentralized reform as mandated by the Individual with Disabilities Act (IDEA, 2004) have resulted in systemic inequities (Rinaldi \& Higgins-Averill, 2011). Furthermore, the government has blurred the lines between two separately conceived policies: the No Child Left Behind Act (NCLB, 2001) and the Individuals with Disabilities Act (IDEA, 2004), the latter of the two having conceptualized interventions differently and thus subsequently changed budgetary allocations (Fuchs, Fuchs, \& Stecker, 2010). An examination of these two regulations reveals a historical timeline that describes a plan that was never developed to adequately address the needs of the most challenged learners (Fuchs et. al., 2010). Because of this lack of follow-through by the federal government, states have come to interpret these 
federal guidelines differently, resulting in more ambiguities and subjectivities.

The blurring of federal guidelines coupled with an individual state's inadequate or even ill-conceived attempts to translate the regulation at the classroom level has resulted in many problems, not the least of which is that is has blurred the educator roles and changed the ways in which teachers and the professional members of a school's staff interact.

\section{Changes in the Education Landscape}

The changes, at least for special educators, began when Congress passed the Individuals with Disabilities Education Improvement Act (2004) which promoted the Response to Intervention (RTI) model as an approved process for identifying students with learning disabilities (SWLD) Some features of RTI, namely universal monitoring and widespread interventions with research-based evidence, originated with the President's Council of Excellence in Special Education, the Office of Special Education Programs (OSEP) and the National Reading Council (NRC) (Kozleski \& Huber, 2010). The emphasis for early intervention in RTI was based on the NRC's report on minority students and their literacy needs (Donovan \& Cross, 2002). These features of RTI were designed to ensure that all students learn using empirically based interventions and certain measures to make this determination (Fuchs et al., 2010).

\section{Response to Intervention (RTI) Models}

The Response to Intervention (RTI) process is based on the premise that if teacher supports are provided early, monitored systematically, and adjusted 
intentionally to respond to individual children's needs, then the more traditional practice of waiting for a child to demonstrate failure would be avoided. RTI consist of five principles: 1) universal screening of all students to identify those who are making adequate progress, at some risk of failure if not assisted, and at high risk of failure without specialized support; 2) data based decision making and problem solving; 3) continuum of evidence-based interventions; 4) continuous progress monitoring; and 5) implemented with fidelity.

According to Beecher (2010), as more schools are implementing RTI, some aspects may require further examination before the process can be used without reproducing the inequalities that plague some current special education practices. RTI has been described as an alternative approach to identifying and providing instruction to students in regular education before they start demonstrating measurable discrepancies that result in a referral to special education. Beecher (2010) states, "In response to the need to identify needs at the earliest opportunity, a teacher must conduct benchmark assessments for determining initial interventions in the classroom" (p. 1).

Although there are common principles that apply to RTI, a typical model is the three-tiered program. Initial screening for all students by a teacher will identify "cut-offs" for targeted interventions in small groups which separate 15$20 \%$ of student population. When monitoring collected data of students, the declining student is moved to Tier III for intensive instruction this accounts for approximately $1-5 \%$ of school population. For $80-90 \%$ of students who progress with above interventions, they are then moved back to Tier I with differentiated 
instruction in the classroom setting (Fischer and Frey, 2005). The third tier is typically reserved for the most specialized instruction being special education.

Beecher's 2011 study "RTI Problems and Possibilities" gives the historical promise of RTI and discusses present RTI models: 1) standard protocol where interventions are prescribed and delivered to all students and not based on individual characteristics (McEneany, Lose \& Schwartz, 2011) 2) problemsolving model in which teams select interventions based on student's particular needs (Fuchs, Fuchs, \& Stecker, 2010) and 3) a hybrid model that incorporates both models (Harlacher, Nelson. \& Sanford, 2010).

Beecher's data suggests the typical RTI model is linear and not "recursive" since many of the students who fail to respond are eventually placed in specialized interventions such as special education (2010). This model is problematic in that it does not give a clear picture of students' opportunity to participate in regular education. Beecher argues RTI should be looked at collectively as a grade performance and not on an individual basis. Also Tiers 1 and 2 are usually addressing behaviors, attendance, and motivation, which are more indicative of sociocultural issues than learning disabilities.

The hybrid model, which uses the problem-solving model approach, is a recursive model. This means a team, consisting of a teacher, school psychologist, special educator, literacy specialist, and administrator, defines a problem and selects an intervention specific to the child's needs. But the problem lies in "Who is defining the problem?" (Beecher, 2010, p. 3.) There is a likelihood the intervention could be inappropriate and more importantly how is the problem 
defined and monitored. The Individuals with Disabilities Education Act (IDEA) does not specify a specific instrument for assessment for RTI. Shinn (1989) and Fuchs, Fuchs, and Compton (2006) agree another important feature to RTI success is the ability for teachers to administer developmentally appropriate assessment instruments in order to implement RTI.

A commentary in "Rethinking RTI at Middle and High School" Fuchs, Fuchs, and Compton (2005) discuss RTI models specific to this population. In their opinion when considering referrals to RTI in the high school, attention must be addressed to responsiveness in RTI program. Unlike the elementary schools, which use the traditional linear model, and the middle school, which uses the quasi-hybrid model, the high school benefits from neither model. The response time in these models is involved and lengthy requiring resources that are not available (Harlacher et al., 2010). Despite RTI's focus on being responsive, policy makers had failed to account for structural barriers and differences in resources. Hence, the implementation of RTI lacked sustainability and created confusion, frustration, and often alienation between general and special educators.

\section{Resources Shift Educator Roles and Responsibilities}

Shifts in resources such as budgetary allocations and changes in responsibilities are the kinds of differentials that alienate and/or create alliances and coalitions (Bolman \& Deal, 2013). With IDEA (2004), the work of special educators, once recognized as the gatekeepers to special education, has been somewhat replaced by a general education initiative called Response to Intervention (RTI) (Fuchs et. al., 2010). As a result of RTI, general educators, 
not specifically trained special educators, now monitor students' progress and intervene as needed with evidence-based practices. IDEA (2004) also mandates that students with a specific learning disability (SLD) are to be taught in a least restrictive environment (LRE). Rather than stigmatizing and separating special needs children, special education and general education teachers have needed to collaborate in variety of co-instruction models to accommodate various learning styles (Fuchs \& Fuchs, 2006).

For speech-language pathologists, this shift from the older, traditional model in which students are taken out of the classroom for special education services to the newer co-instruction models was not easy. In a mixed service model, confidence and trust among team members is important (Russell \& Kadareravek, 1993). In a slightly different but still relevant example of the problems a mixed service model can engender, Magnotta (as cited in Beck \& Dennis, 1997) found that SLPs found the transition from medical worksites to educational models challenging since they felt they lacked expertise in classroom settings. Likewise, Fallon \& Katz (2011) found SLPs felt they were ineffective in the classroom and were not adequately instructing students with reading and writing issues and they attributed that dilemma to ASHA's broadening their scope of practice. Beck and Dennis (1997) reported educators felt SLPs were less competent with the training provided on class-based intervention strategies.

The American Speech-Hearing and Language Association (ASHA), speech-language pathologists' national professional organization, recommends that SLPs assist and partner with other school personnel in the provision of 
services for reading and writing. Since reading comprehension combines word recognition with listening comprehension, SLPs' contributions to literacy include their knowledge of sound and symbol associations (phonemics), a complex process involving a student's ability to integrate auditory and visual modalities (Gough \& Tummer, 1986; Adams, 1990). In fact, without explicit instruction, phonemic awareness eludes $25 \%$ of middle class first graders and even more of that group of students from less literate-rich backgrounds (Adams, 1990). Also important to phonemic awareness is phonological looping, which is a form of working memory whereby one retrieves a sound to match a symbol (Farqhuarson, 2012; Cabbage, 2016).

At the middle and secondary levels, students' academic demands increase with complex vocabulary and length of written and spoken expressions, making phonological looping more complex since working memory also factors into a student's ability to assemble sentences of increased length and complexity (Nippold, 2016). Studies showed that with speech-language intervention, a student's use of relative clause production increased during adolescence and into adulthood, along with their mean length of utterances and vocabulary (Nippold, 2016; Nippold, Ward-Lonegan, \& Fanning, 2005). Although literacy research suggests that SLPs should be involved in literary interventions, other research indicates that SLPs feel ineffective in the classroom. Their feelings of ineffectiveness and incompetence needs to be better understood and addressed because students with speech and language issues are now being monitored in the general education setting. 
In response to these causal effects and links between language and literacy, some speech-language studies suggest new ways of monitoring the macrostructure and microstructure in language progress (Gillam \& Justice, 2010) and of narrowing the literacy gaps through a content continuum framework (Ehren, Deshler, \& Graner, 2010). SLPs are analyzing student narratives at two levels: macrostructure, which includes students' skills in initiating, assembling, and sequencing episodes ( Gilliam \& Ukrainetz, 2006) and microstructure, which includes students' abilities in using conjunctions to join dependent and independent clauses (Liles, Duffy, Merrit, \& Purcell, 1995). Content Literacy Continuum (CLC) (Lenz, Ehren et al., 2010) is a framework that was designed for organizing schoolwide literacy efforts in middle school and junior and senior high schools. The CLC framework aligned interventions across school levels; it also facilitated a process alongside the analysis of language structures to better monitor student growth and responsiveness.

Other research (Werts, Lambert, \& Carpenter, 2009; Swanson, Solis, Cuillo, \& McKenna, 2012; Pyle, 2011) suggests that special educators and general educators have different ways of understanding roles, assessment protocols, and practices associated with RTI initiatives. Following a survey on the implementation of RTI, there was confusion among staff as to the roles they would have, i.e., to collect data and interpret results. Of note, the participants in the study could not come to a consensus on whether the discrepancy model should be retained or abandoned (Werts et al., 2009). While Werts et. al. (2009) examined general and special educators' perceptions of barriers, another study of 
special education directors' perception of barriers to RTI reported similar issues pertaining to role conflict (Sanisosti, Goss, \& Notlemeyer, 2011). The implementation of RTI offers an opportunity for special education directors to emerge as agents of change to "promote collaboration between general and special education teachers ... to assure that high quality education programs are accessible to all students" (Lashley \& Boscardin, 2003, p. 3). However, the extant literature does not adequately address how to build and sustain the educational practices that promote a merger between general and special education practices within the context of RTI (Sanisosti et al., 2011).

\section{Conflicting Structures, More Ambiguity}

Response to Intervention exists along with many multi-tiered intervention systems; however, many of these systems lack scientifically based protocols (Fuchs et al., 2010). Currently there are no guidelines or recommendations for empirical interventions. Furthermore, there is no language in NCLB that explicitly allows or requires funds for these systems. Yet, administrators are allowed to use $15 \%$ of Title I money for funding reading programs within the RTI system (Kaloi, 2012). Making this more explicit is important because it is NCLB, not IDEA (2004) that is the authorizing law for Title I money. Title I provide funds for staff, training, and resources for students struggling in reading and math. Although some Title 1 legislation includes funding for evaluating interventions, it is rarely enough to assess the intended effects and, moreover, IDEA (2004) and state legislation rarely provide such funding (Allington, 2002). In order to make NCLB and IDEA (2004) more congruent to ensure funding for struggling learners, it is 
important that NCLB includes specific language that governs the use of Title I funds for RTI. Authorizations are continuously being debated as to which practices and reform models are scientifically proven and subsequently play a major role in the distribution of federal funds (DeBray, 2006; Popham, 2008).

Race to the Top (RTTT) was part of the American Resource and Recovery Act during the Obama Administration and shared some of the principles of NCLB (Vittieri, 2013). In RTT, the government awarded states money that was supposed to address problems with previous reform. Despite criticisms regarding past accountability measures, RTTT emerged with a higher set of standards, i.e. teacher evaluations based on student performance and adherence to common core curriculum. Many believed that the problems with reform were due to funding distribution and reliance on competitive funding. For instance, states had to meet a set of conditions that had assigned point values, and state's with higher scores were given money and the higher the score, the bigger the grant. Incidentally, some of those conditions resembled Secretary Duncan's recommendations for NCLB's Annual Yearly Progress (AYP) (Vitteriti, 2013). RTTT was flawed in two ways, first, the states with capabilities and resources were awarded grants; and second, a higher set of standards was not effective due to variable and inconsistent designs (Vitterirt, 2013; Fuchs et al., 2010). As such, the achievement gap between rich and poor widened and high school graduation rates decreased (Vittieri, 2013).

Therein lies the problem in reform: the inconsistencies, incompleteness, and ambiguities inherent in policy making make it difficult to implement effective 
and sustainable programs. Existing studies stress the importance of empirical and research-based practices; however, research fails to address what is beneath the inconsistencies and ambiguities. This study attempts to delve into ambiguities with which SLPs contend as they serve their students.

\section{Structures that Impact the Response-to-Intervention}

Four issues impact the effective delivery of RTI: system/logistics structures as barriers; the need for changes of roles and attitudes; the lack of evidenced-based practices; and the lack of training and professional development (Sanisosti et al., 2011). Logistical issues predominantly impact the delivery of evidence-based practices, job description and performances, and types of professional development when schools are working with different sets of criteria and different budgets that impact resources (Werts Lambert, \& Carpenter, 2009). Such differentials have an impact, such as could be seen in a 2008 national survey on RTI implementation in which 44 states $(88 \%)$ were only partially implementing RTI (Hoover Baca, Wexler-Love, \& Sanchez, 2008). A more recent survey in 2011 found that three years later only $23 \%$ of the states were in full implementation (Cramer, 2015). In both studies, there was found to be a lack of fidelity in monitoring student responsiveness in RTI (Werts, et al, 2009). Likewise, Pyle (2011) reported on a pilot study implementing RTI in which progress monitoring was inconsistent. Based on those findings, Pyle (2011) recommended professional development that addresses the interconnectedness of fluency and comprehension and how skills can be taught in a cohesively and monitored uniformity. 
A study by Madda, Halverson, \& Gomez (as cited in Pyle, 2011) found the "plethora of initiatives and incommensurable goals" burdensome therefore restrictive to RTI's development (p. 70). This review found many quantitative studies that showed how inadequate training and monitoring systems are impacting successful implementation of RTI. According to one survey, general and special educators have little consensus on their roles and eligibility criteria for referring students to special education services (Werts et al., 2009). Lacking are qualitative studies that examine special and general educators' responses to RTI. More studies on participants' perceptions and agency would add to this literature on how they might negotiate disagreements and inconsistencies in their practices that have implications for their students and profession.

Some monitoring of the RTI model does occur. At the elementary school level, the RTI model is linear as teachers use frequent data collecting for their intervention planning; however, at the secondary level, the RTI model is recursive, which means that a problem-solving team must meet to define the problem (Beecher, 2010). Inconsistencies among the categories of criteria, practices, and teachers' thinking between a district's elementary and secondary levels counter the benefits and purpose of RTI (Pyle, 2011). RTI research studies are mostly small-scale at the elementary school level (Fuchs \& Fuchs, 2006; Pyle, 2010; White, Polly, \& Audette, 2011) and do not translate well to large-scale applications (Reynolds \& Shaywitz, 2009). 
According to Tilly (2003), many agree that RTI is a conceptually-sound model; the flaws lie in the implementation (as cited in Reynolds \& Shaywitz, 2009). Other research supports inclusionary instruction, which is common in the RTI model. However, a recent study in Virginia surveyed SLPs' delivery models at the secondary level. Despite the evidence-based benefits of RTI and inclusionary practices, SLPs preferred the IEP model over the RTI model (Salley, 2012). The traditional model was preferred because of the secondary schools' non-collaborative environment, which SLPs attributed to overloaded schedules (Salley, 2012). In another survey, special education directors reported that logistical barriers such as scheduling made the implementation of RTI difficult (Sanisosti et al., 2011; Brozo, 2010). Additional studies individually focused on secondary school levels, such as Salley’s 2012 study, are necessary in order to attain more solid and empirical research on this subject that varies across grade levels (Reynolds \& Shaywitz, 2009; Sanger, Brunken, Snow, Ritzman, 2012; Brozo, 2010).

\section{A quandary: rethinking the RTI framework at the secondary level.}

Based on the research reported above, it is clear that two models of RTI, linear and recursive, sometimes exist within the same school district at different levels. Without a separate study of each level, reliable growth data of middle and high school students is difficult to ascertain (Beecher, 2010; Shinn, 1989; Brozo, 2010). According to Fuchs, Fuchs, \& Compton (2012), RTI designs in schools need to be rethought because broad interventions are expensive and unnecessary 
and Reynolds \& Shaywitz (2009) added that the screenings inadequately monitored needs of secondary students who need specialized instruction. Fuchs et. al (2012) reported on specific monitoring systems called Smart RTI which used multi-stage screening and found students were more responsive. However, according to Brozo (2010) such screening processes are not compatible with secondary school's schedule nor do they align with secondary curriculum.

RTI screening systems, which are mostly focused on reading interventions, have come to associate only reading deficits with most LDs and do not include the problem of language impairments (Brozo, 2010; Kavale, Holdnack, \& Mostert, 2005). This is problematic for the adolescent with challenges in thinking processes and working memory who now struggles with reading across subject areas, which is a more prevalent aspect of the secondary education level than the elementary one (Tovani, 2000; Cabbage, 2013; Nippold, 2017). According to Allington (2002), the current types of intervention for older, struggling readers are not effective; what educators teach to elementary students is not what should be taught to these older, challenged readers. To complicate matters, middle and high school teachers are not required to take literacy instruction as often as their elementary counterparts (Deshler, Palincsar, Biancarosa, \& Nair, 2007).

Relatively little has been written about monitoring students' language deficits under the RTI model despite the fact that language is intrinsic to reading and is a hallmark feature of learning-disabled students (Gilliam \& Justice, 2010). 
Speech-language pathologists remediate language disorders. They report that morphemes are the key to unlocking pronunciation, spelling, and meaning for big words (Adler \& Van Doren, 1992, p. 126). According to Merriam Webster, morphemes are a "distinctive collocation of phonemes (such as the free form pin or the bound form $-\mathrm{s}$ of pins) having no smaller meaningful parts. Morphosyntax knowledge contributes to vocabulary (Catts \& Kamhi, 2012) and has relevance when readers can compare new words with ones that they already know that contain the meaning of the new words but with their differing grammatical constructions. According to Nippold (2017) and Carlise (2010), students' knowledge of morphemes increases throughout the elementary and adolescent years. Since the brain is malleable and younger brains learn to adapt by using alternative pathways, it is possible that reading can continue to improve with recognition and chunking of information into patterns. Therefore, harnessing the SLP's expertise in the neuroscience of speech and English language phonemic inventory is essential in remediating vulnerable groups, as is the case for students of this study's SLPs who are impacted by the nine-year rule.

\section{Implications for Students and SLPs}

Generally speaking, speech-language pathologists consistently attempt to help students with unique literacy needs and backgrounds, and often have to work with rules that may prove challenging. However, unlike any other state, Rhode Island's SLPs must follow the dictates of the nine-year rule in schools while complying with the RTI model within their school administrations. For example, 
RTI is a general education initiative whereby 70 to $80 \%$ of the student population receives Tier 1 instruction that is implemented by general educators, not SLPs (Shapiro, 2012). As such, students with a history of speech services are not necessarily being properly addressed. Typically, in a traditional RTI model, the school population is divided into three tiers of instruction (White et al., 2011; Reynolds \& Shaywitz, 2009; Martinez \& Young, 2011). Before RTI and the introduction of the nine-year rule, SLPs created an Individualized Education Plan (IEP) for students with speech-language issues with detailed speech provisions in terms of short- and long-term goals, types of interventions, as well as the frequency, placement, and duration of said interventions. Students could conceivably continue to have speech services throughout the high school years. Speech-language pathologists had to rely on their broad scope of knowledge in language development, human cognition, and education to determine student needs and eligibility for these specific speech-language services, which was done on a case-by-case basis (McNamara, Hindenlang \& Cascella, 2004; Al-Mondhiry, 2008). The new regulations have compromised SLPs' expertise, experience, and agency in identifying what students need specialized services and for what reasons at the expense of these students.

\section{Students with Speech Sound Disorders}

Students who are now discharged from SLP services due to the nine-year rule are mostly diagnostically characterized by speech sound disorders (SSD), which manifest differently across school-age years when compared to the typical 
learner. Speech sound disorders can occur at any age and include phonological disorders (processes of speech productions), articulation disorders (one to two sound distortions), neurogenic/congenital or acquired aphasia, apraxia, and/or dysarthria, and speech impairments secondary to structural and/or sensory deficits, e.g., students with cleft palate and/or hearing impairments. Although multiple factors contribute to reading success (Robinson, 2005), Moats (as cited in Cabbage, 2016) believes that students with language-based problems can fall anywhere on the spectrum, making predictability more complicated. Moreover, students with Speech Language Impairments (SLI) have underlying issues that impact their spoken expression and manifest in their writing in later years.

Therefore, SLPs' are justified in their concerns about students lagging in spoken ability (Gilliam \& Johnston, 1992; Gilliam \& Ukrainetz, 2006). There is little research that examines developmental factors and the impact of an SSD and SLI in relation to reading disorders in the adolescent years (Brozo, 2010; Skebo, Lewis, Freebaim, Tag, Ciesla, Stein, 2013; Sanger et al., 2012).

\section{Speech errors persist in adolescent literacy development. Persistent}

errors in speech sound production are present for adolescents with a history of speech sound disorders (Lewis, Freebarin, Tag, Ciesla, Iyengar, Stein, \& Taylor, 2015). The cause of those production errors is not clearly known, but there are possible links to either poor phonological representation of words or oral-motor abilities. For example, one study found that many participants with histories of speech and language disorders continued to make articulation errors at 18 to 20 
years of age (Johnson, Beitchman, \& Brownlie, 2010). Flipsen (2002) theorized that 12- to 16-year-old students with markedly decreased rates of speech were actually compensating for sequencing issues. Other studies have reported that adults with a history of SSD committed more sequencing errors on multisyllabic and nonword repetition than did adults without a history of SSD and exhibited slow rates on tasks involving sequential alternating compared to repetitive movements (Peter, Button, Stole-Gammon, Chapman, \& Raskind, 2013). Although many of these errors were minor distortions and did not impair intelligibility, findings suggest deficits at several levels of processing, including encoding, memory, and/or translation into motor acts (Peter et al., 2013). These several levels of processing, i.e., phonological processing that results in speech errors and slowness in articulation, are also involved in word recognition and sound-symbol associations and can negatively impact oral and written literacy skills (Lahey, 1998; Nippold, 2017).

\section{Outcomes and Shortcomings of Authorizations}

Since the National Report Card (2005) reported that 27\% of eighth-grade students were reading below basic levels and only $31 \%$ were performing at grade reading level, schools have been focusing on literacy programs in the secondary level (Paulson, Koester, Mell, \& Nielson, 2009). Speech-language pathologists and literacy specialists have created a continuum literacy framework for middle, junior, and high school students that embeds instruction across content areas (Ehren et al., 2010). For instance, in the state of Montana and the city of Chicago, collaborations between SLPs and educators have resulted in new monitoring 
instruments and training protocols (Paulson et al., 2009; Ehren, Lybolt, \& Gottfried, 2010).

Monitoring Systems. The Dynamic Indicators of Basic Literacy Skills (DIBELS) has been the most commonly used universal monitoring system. However, DIBELS does not typically measure accuracy in oral fluency, which is an important indicator in adolescent reading success (Gilliam \& Justice, 2010). The current decoding- and fluency-focused RTI models do not adequately monitor oral development. As a result, the language needs of this new population of RTI-identified at-risk readers may be overlooked, particularly subgroups that may appear to have resolved their initial difficulties ( Allen, Ukrainetz, Carswell, 2012) Now SLPs are monitoring language with samples of contextualized and decontextualized narratives that provide information about micro- and macrolinguistic structures and have been useful in designing reading interventions (Gilliam \& Justice, 2010; Ehren \& Deschler et al, 2010; Ehren \& Lybolt et al., 2010; Paulson et al., 2009).

Barbara Ehren (2000), a speech-language pathologist with expertise in literacy, has suggested ways to address some of the problems surrounding the latest shifts in education. She suggests that school-based SLPs: (a) accept new or expanded roles with curriculum in general and literacy specifically; (b) provide curriculum-relevant therapy to students and assistance to teachers; and (c) design and implement delivery models based on the nature and setting demands of school, the teaching realities, and the challenges faced by secondary school students (Ehren et al., 2010). 


\section{Relations and Perceptions Matter to SLPs' Work}

Generally, an abundance of research addresses specific issues related to the speech-pathologist's' role: interventions/training (Gilliam-Justice, 2011); delivery service models (Ukrainetz \& Fresquez, 2003); and caseload management (ASHA school surveys, 2008, 2010, \& 2014; Katz, Maag, Fallon, Blenharn, \& Smith, 2010). Shifting roles and aspects of collaboration continue to be associated with policy. For instance, research on the collaborative and co-teaching model was stimulated by changes in specific learning disability (SLD) criteria and mandates, such as the least restrictive environment (LRE) and RTI (White et al., 2012; Ukrainetz \& Fresquez, 2003; Sanger et al., 2012). However, surveys about attitudes, perceptions, and satisfaction are also important in understanding the SLP role (Elksnin \& Capilouto, 1994; ASHA school surveys: 2000, 2008, 2010, $\& 2014)$.

The measure of general teachers' perceptions of SLPs affect SLPs' own valued perceptions (Ritzman \& Sanger, 2007; Ukrainetz \& Frequez, 2003). Those perceptions factor into SLPs' interpretations of their value and recognition. For example, a 2009 survey from Georgia (Consolini, Carson, Miller \& Johnston, 2009) suggests that meetings, responsibilities, and paperwork related to RTI prevent SLPs from serving some IEP students. Since their workload is often not accurately reflected with having caseloads and workloads, SLPs assume their colleagues believe they do not have many students in their caseloads (Consolini et al., 2009). Moreover, SLPs attributed lack of referrals of students' vis a vis RTI were in many cases due to the general teachers' aversion to the lengthy and 
inefficient RTI process, which SLPs had already declared as a detriment to the success of their work with their students (Consolini et al., 2009). Such perceptions can potentially result in LD students being further academically compromised when they are not referred for services.

\section{Influences Behind SLPs' Job Satisfaction}

Professional identity development is limited to a self-image that permits feelings of personal adequacy and satisfaction (Paterson et al., 2012). Clouder (2005) conceives of another professional identity constructed around caring for others. Critical theorists, Boler (1999), Noddings (2005), and Belenkey, Clinchy, Goldberger, \& Tarule (1997), would concur that caring as well as nurturing and reciprocity contribute to the identity process. While ASHA's surveys on job satisfaction illustrate job trends in the speech profession, they do not explain the influences behind job satisfaction and their relationship with one's caring and feelings. The following literature acknowledges the importance of these influences alongside one's self-image in shaping their professional identity.

In the 1980s, professional isolation and personal ineffectiveness were cited as sources of stress and dissatisfaction for SLPs working in public schools (Wisniewski \& Gargiulo, 1997). More recently, ASHA has conducted large-scale surveys of SLPs' perceptions of work conditions in public schools (ASHA, 2004, 2018). In the early 2000 surveys, SLPs responses to questions about work challenges and the results paralleled the 1980s results, which noted the following challenges: excessive paperwork, large caseloads, and not having enough planning time (ASHA, 2004). In 2018, ASHA's survey design was different in 
terms of the questions that were centered on ASHA's performance and not the SLPs' working conditions. SLPs favorably reported on ASHA's support in providing evidenced-based resources; however, advocacy on behalf of SLPs was less favorable. Although surveys of large numbers of SLPs have provided broad descriptions and quantitative summary data on SLPs practices (ASHA, 2000) they do not reconcile findings (Giangreco et al. 2000). Therefore, issues such as contradictory service models, overlapping roles, and a lack of advocacy on behalf of SLPs challenge their practice and often go unexplained and uninvestigated.

In response to reform and role changes in SLP practices, ASHA, which had previously looked at mandated caseloads, began to examine the clinician's entire workload, a term defined as all activities that are required and performed by and with SLPs, as well as details concerning his or her professional relationships (ASHA, 2002d, 2002e). ASHA's individual state and national surveys have consistently cited large workloads, excessive paperwork, and a lack of collaboration as sources of its members job dissatisfaction (ASHA, 2004, 2008; Consolini et al., 2009; Fuchs et al., 2010). SLPs reported being worried that their jobs were at risk because education mandates that had formally funded special education personnel and programs were now funding a general education initiative, namely RTI, instead (Consolini et al., 2009; Fuchs et al., 2010). More recently, ASHA has also examined job trends - a 2016 survey on workforce interests shows a 20\% decrease in job openings since 2008 (ASHA workforce, 2016). Perhaps SLPs' feelings of worry about job security correlates with education policy. As a result of these tensions, it would appear that SLPs' 
perceptions of job security combined with their lowered levels of job satisfaction have implications for the way in which they navigate tensions.

\section{SLPs' Perceptions of Value in Co-taught Classes}

Beck and Dennis (1997) conducted a survey of open-ended questions for SLPs and teachers. The results indicated that both preferred the co-instruction model, yet it was less frequently used. SLPs and teachers also agreed that training for the newer job demands was lacking and that SLPs were not involved in common planning time (Beck \& Dennis, 1997). Likewise, another survey found that time constraints contributed to SLPs' reasons behind relinquishing one third of their caseload in a co-taught class (Sanger et al., 1999). Given IDEA's (2004) push for a focus on curriculum, the co-instruction model is generally recommended in schools (Harn, Bradshaw \& Ogletree, 1999). Despite teachers' and SLPs' consensus in that 1997 survey, SLPs' perceived classroom instruction did not adequately facilitate remediation of speech goals (Beck \& Dennis, 1997). Implications from that study suggested that teachers and SLPs would benefit from conversations on how to facilitate each other's role in the classroom.

\section{Professional Identity in Higher Education}

There is literature on the development of professional identity in higher education; however, higher education programs, such as Communication Disorders programs, appear to be lacking pedagogies and curricula related to professional identity development. Work-related issues alter one's identity and disposition when it contrasts with one's formal training. "Higher education programs are required to produce graduates who display mastery of theoretical 
ideas and knowledge competencies, applying theory and knowledge in their workplace, and professional dispositions that foster ethical and reflective practices" (Trede \& Loftus, 2010, p. 1). Universities are under pressure to develop pedagogically based curricula that ensure that mastery (Reid, Dahlgren, Petocz \& Dahlgren, 2008). However, the literature shows that universities predominantly focus on formal knowledge and fail to debate power and structural influences that result in tensions between personal and professional values (Reid et al., 2008).

The following studies offer scenarios and explanations in which identity work in education is happening. Some of the studies show how perception, value, and recognizability contribute to one's personal adequacies, satisfaction, and selfimage; all of which matters to professional identity development and practices.

\section{"What is Language": A Qualitative Study on SLP Role}

According to Crane \& Iwanicki (1986), role ambiguity and conflict can contribute to burn-out. In seeking to understand the manifestation of burnout and job dissatisfaction, Ukrainetz and Fresquez (2003) chose a qualitative research paradigm that coded interviews of SLPs and reading and resource teachers. The interviews covered topics such as speech-language practices in comparison to the practices of reading and resource teachers. The categorical findings showed that these three professional groups shared three areas of concern: job pressures, service delivery, and underlying instruction frameworks. The three groups also agreed that they all provided language instruction; however, the SLPs' definition of language differed from that of the teachers. Of note, one SLP discussed her reluctance to identify herself as a speech-language pathologist, stating the 
following: "You go "speech language pathologist," [and] all of a sudden you've somehow elevated yourself educationally and socially above these people that you really badly need to work with..." Instead, she identified herself as a speech teacher. Another SLP stated, "With all the current emphasis on standards and benchmarks, it is difficult to decide if I teach reading and writing — where is my territory and where is the teacher's?" This SLP questioned whether or not the current educational model attached any importance to a student's need for the development of intelligible speech and vocabulary. Although this study helped to reconcile instructional differences in language and literacy by using teachers' perspectives, the manner by which they changed or resisted roles and practices was not sufficiently explored.

\section{Teacher Identity Work and Figured Worlds}

Figured worlds are rules that influence the way people speak, act and practice within social and institutional spaces and in this study, SLPs encountered differences in an educational model and accessed figured worlds in assuming a teacher identity (Holland et al, 1998). Danielewicz (2001, p. 4) writes about teacher identity work: 'I regard 'becoming a teacher' as an identity forming process whereby individuals define themselves and views by others and teachers." Clarke (2005) conducted a mini-case study in which one teacher shared web notes on how he negotiated a change in his identity from one who did not like confrontation to one centered on keeping challenging students on-task with motivating lessons. Clarke (2005, p. 22) argues that: 
"In order to understand language teaching and learning we need to understand teachers: the professional, cultural, political and individual identities which they claim, or which are assigned to them'. Overall, we can say that identity is increasingly being seen as a crucial component determining how teaching and learning are played out in schools and classrooms.

Many of the dilemmas that SLPs face come about in part due to their conflicted identities in light of how their school's teachers perceive their role (am I a teacher or a SLP and how does that impact my standing within the school?). Much of the research on the SLP role and interactions demonstrates that SLPs carry consistent burdens reflected in emotions, i.e. worry, dissatisfaction, burn-out, and the need to be valued by others.

\section{Social Recognition in SLP Identity}

Identities are negotiated through social recognition, which is accomplished through perspective taking, workings of institutions, and affinities with certain groups (Gee, 2008). Moreover, recognizability is achieved when one dresses, talks, and acts a certain way, through and by one's demonstrated feelings and values and use of certain artifacts and tools. For instance, in the aforementioned study titled "What is Language?" school standards and benchmarks constrained SLPs' practices. It also changed who they were recognized by and how they were recognized; as such, they asked, “Am I a teacher or a pathologist”. SLPs negotiated a teacher identity knowing they needed a working relationship with the classroom teacher. Although that study was qualitative and reconciled 
instructional needs, it did not discuss the negotiation process of identity formation nor the value of social recognition.

That sort of negotiation is central to SLPs' agency in their advocacy of students with unique needs. There appear to be few studies that have delved into the interrelatedness of a school's teachers with its SLPs. Studies have not determined how SLPs' identity is formed on the basis of recognition and value within that group and how this affects and is bound up with the preservation of an identity based at least in part on their ethics, their mission and practice.

Many studies on RTI emphasize collaboration and "buy-in" (Fuchs, Fuchs \& Hollenbeck, 2007; Fuchs et al., 2010), thus there is value in examining studies that look at other stakeholders' perceptions, the structures that they bring, and how they intersect with each other. In one study, feedback after a year-long implementation of RTI found that 54\% of secondary general educators were dissatisfied with SLPs (Sanger et. al., 2012). Ritzman \& Sanger (2007) conducted a study that found educators' opinions of SLPs less favorable in the co-class instruction model, reporting that SLPs lacked classroom management skills. On the other hand, they reported that principals had favorable opinions of SLPs who brought unique diagnostic skills for complex cases (Ritzman \& Sanger, 2007). According to the findings of a qualitative study (Sanisosti, et al., 2011), study participants agreed that shared perspectives make a difference in maintaining components of RTI. A study that examines value from the perspective of stakeholders and speech and language pathologists' perspectives can make a difference for intervention and practices on behalf of certain students. 
Recognition work is essential to agency and identity. This literature review includes SLPs' evaluations of a service model (Harn et al., 1999; Elkinsin \& Capilouto, 1994; teachers' and SLPs' opinions on classroom-based interventions (Beck \& Dennis, 1997); and SLPs' and teachers' ratings of delivery service models (Ukrainetz \& Fresquez, 2003). Most reported SLPs' speech time as lacking, thereby impacting training and planning. Another study compared parents and SLPs' evaluations of a service model (Bellon, Vereen, \& Ogletree, 2001). Although participants agreed time and training are closely related problems, the SLPs attributed poor communication with the classroom teacher, while parents believed that SLPs were not properly supported (Beck \& Dennis, 1997; Bellon et al., 2001). Ultimately, how SLPs, in integrated settings, choose to be recognized by perceived value is an underexamined area and their navigation of those tensions are important to agency and identity.

These studies highlight differing vantage points and reveal how their explanations differ but do not explore how SLPs must improvise and negotiate the tensions they naturally experience when school culture, education policy, and professional and personal aspirations intersect.

\section{Conclusion}

In this chapter I reviewed certain aspects of federal and state education that relate to the work of speech-language pathologists; the shift in general and regular education resulting in role ambiguities and conflicts for these professionals; the general implications for certain students and the SLP profession; and sociocultural aspects that shape SLPs' perceptions as supported 
with the literature on identity work. In the complex and dynamic process of negotiating dilemmas posed by policy and positions of stakeholders and administrations, SLPs' perceive their value as based on others' perceptions of them, which often results in dissatisfaction and frustration on the part of SLPs as well as their general teacher counterparts. Unfortunately, research that delves into attitudes and perceptions within the education field is generally lacking and in the case of Rhode Island, these variables have not been studied at all. While research on identity work, which includes the area of social recognizability, has been the topic of some studies, they have mostly addressed the general education population. This study addresses the lack of research on identity and agency in general and specifically for Rhode Island's SLPs considering their constraints.

In contrast to the above topics, there has been an abundance of current research on outcomes of education policy, types of language disorders and literacy instruction, and teacher identity work, yet none of it explains how and why SLPs have come to endorse and implement a rule that they believe is inadequate and perhaps even detrimental to their students. Since speech-language pathologists provide services for vulnerable populations, they can be viewed as institutional agents in assisting students and their families in accessing and advocating for appropriate services (McQuat, 2007). However, when SLPs do not improvise ways to accomplish their work in the face of a rule that opposes this role/identity and instead endorse a rule that they consider to be unfair, this passive acceptance causes personal and professional dilemmas. It is crucial that there are available studies that inform SLPs, school leaders, and policymakers about the 
factors that have contributed to this problem. As of this date, there has been no study on Rhode Island's nine-year rule. Only the state's professional organization for SLPs has attempted to learn more about it and only through one survey.

This study addresses a significant gap in the literature by applying a critical stance to how SLPs negotiate tensions related to policy enforcement and to promote positive change for a vulnerable population. To get an in-depth understanding of SLP identity work across their settings and experiences, I designed a qualitative research study that applied a critical discourse analysis of their social and linguistic practices. In so doing, I have attempted to demonstrate how SLPs in Rhode Island have responded to challenges and tensions in their work that originated from the interaction of education policies that did not and still do not align with their professional standards and preferred practices. 


\section{CHAPTER 3: METHODOLOGY}

In this chapter, I will first provide an overview of the methodological paradigm within which this study is situated. Next, I will outline the procedures used to collect and analyze data. Finally, I will provide a rationale for my choice to transform the findings into schema explaining speech- language pathologists' agency and identity.

\section{Naturalistic Paradigm Situated in My Study}

A researcher's theoretical lens often informs the methodological approach. In this study, the critical theoretical perspectives are rooted in culture, politics, and economics. I used this critical lens to 1) identify the normative discourses of Rhode Island public school speech-language pathologists (SLP) operating under conflicting policies; and 2) to critique the implications of these conflicts for this profession and a subgroup of students (Patton, 2012; Guba \& Lincoln, 1994). The epistemological focus of any critical study is subjective in so far as facts can never be separated from values and ideologies and all thought is mediated by historically and socially constituted power relations (Guba \& Lincoln, 1994). As such, during the interview process, in which SLPs recounted their experiences, I kept a reflective journal to keep an account of any subjectivity on my part that would affect the analysis of the data.

\section{Critical Perspective: Subjectivities and Language}

A major tenet of critical theory is that language is central to the formation of subjectivity and identity (Gee, 2014). In 2016, an IRB approved pilot study was conducted on Rhode Island speech-language pathologists' responses to a 2015 
survey on an education rule. As a result of that study, SLPs' written comments were organized into themes surrounding a rule that conflicted with SLPs' mission and beliefs. This social inquiry study sought to determine how SLPs in a Rhode Island public school responded to the dilemmas that arose as a result of their mandated responsibility to implement a controversial state rule that impeded their ability to maintain their professional standards. Since subjectivities are enmeshed in discourse, I decided to analyze the social and linguistic practices of these SLPs through the lenses of self-perception and their understanding of how others perceived them by examining how they responded to the relevant dilemmas through the language that they used when speaking about their roles at work.

According to Gee (2014), identity is the "kind of person" one is recognized as "being" at a given time and place, can change moment to moment, and incidentally, can be unstable or ambiguous (p. 2). In this study, identity is defined as the role that develops from various sources of power, which may be inherited, traditionalized, affiliated, and/or rationalized alongside cultural and sociohistorical influences (Gee, 2001). Establishing a professional identity requires integration of background with the norms of the profession alongside work experiences. According to Gee (2001), agency is defined as the possibilities and opportunities through which SLPs' identities are recognized. A change in agency establishes a better-defined identity, both on the part of the individual and from the vantage point of others.

Discursive practice is the medium that either enables or constrains agency (Gee, 2001; 2014; Rogers, 2011) and, it follows, identity. Both are transmitted 
through social interaction (Gee; 2008). Therefore, in this study, it was crucial to conduct a method using interviews to secure data that would be conducive to discourse analysis and focus groups to provide rich context. In addition, according to Fowler (2014), focus groups have agentive potential in that they allow for professional conversations in a more social setting, another reason these meetings served the study. I also conducted face-to-face interviews in order to delve further into issues that arose in the focus group discussions that were surprising, confusing, or unexpected. According to Creswell (2014), these issues inform the codes that emerge.

\section{Rationale of Standpoints}

Because this study focused on a discourse of issues such as power-related abuses and entangled systems of power in the world of speech-language pathologists, it followed the tenets of Critical Discourse Analysis (CDA), which investigates discourse and power, specifically the power of social groups and institutions. To gather the appropriate data for this type of research, the study made use of naturalistic inquiry research, which is based on data that is gathered from observing people's behavior and/or conversations in their natural setting (Frey, Botan, \& Kreps, 1999). Lincoln and Guba (1985) maintain that naturalistic inquiry has the benefit of data collection without the manipulation of the environment. Patterns, themes, and categories necessary for analysis emerge from within that natural environment (Patton, 2002).

I am also a speech-language pathologist working in Rhode Island and, like

the SLPs in this study, I believed that the rule in question was causing many 
problems. Therefore, it was necessary for me to maintain transparency about my shared values and beliefs about the patterns and themes that arose from the focus groups. Therefore, I disclosed my position about my frustration and dissatisfaction with this rule. Since I worked with some of the SLPs, it is likely they were already aware of my feelings. However, I wrote analytic memos and notes to acknowledge subjectivities that could perpetuate, obscure or exacerbate power differences (Nygreen, 2013). As an additional precaution to prevent any subjectivity on my part from conflicting with the reporting of the findings, I chose to analyze the data using coding steps and CDA as it provides a systematic approach to interpreting subjective data.

According to Patton (2002, p.177), the following principles undergird qualitative inquiry: action, equity, empowerment, emotions, reflexivity, processes, community and people as understood in their context, and changed processes. For this study, three of these principles were most salient: equity, emotions, and empowerment. Hence, Boler's Pedagogy of Discomfort alongside critical theory were appropriate in framing a study that warranted a social justice perspective. Qualitative researchers must be personally involved and show respect and concern for others by learning about them, their perspectives, and their world (Patton, 2002). As a speech-language pathologist I am concerned about the integrity of the SLP profession and how its members participate and carry out the regulations governing it, which, by definition, impacts a vulnerable population. Therefore, as a participant in this natural inquiry process, there were several considerations that I had to take into account: first, that my beliefs would not necessarily mirror those of 
the interviewees and second, that I needed to ensure that my participation would not influence or shape the participating SLPs' responses, i.e., recruit others to my way of thinking (Gee, 2008). For these reasons, I constantly checked in with my advisor, an established critical researcher, to review the ongoing focus groups' discussions.

\section{Basis of this Inquiry and Design}

According to Denzin \& Lincoln (1994) qualitative research is inquiry that involves a variety of empirical tools: case studies, experiences, focus groups/interviews, observations, visual text; all of which describe routine, and problematic moments, and meaning in individuals' lives. In this study, I conducted two focus groups and face-to-face interviews and applied coding and critical discourse analysis to better understand the formation of SLPs' identities. Denzin and Lincoln (1985) state that research methodology is determined by the nature of the research question and the participants being examined. Foss and Walters (2007) note that research questions inform the coding process so that the researcher can better define and study the lives of their participants. Using this approach, I was able to coherently code and assemble explanations to the research questions. The research design answered the following research questions through description and analysis whereby three categories emerged: systems, complications, and dilemmas.

(1) What were the challenges and tensions do Rhode Island Public School SLPs experience in delivering speech services for students targeted by the nine-year rule in relation to $R T I$ and how do they respond?

(2) What experiences and values do SLPs perceive as significant in the formation of their professional identities?

(3) How do SLPs integrate their values, ethics, professional experience and background/education with the norms of the profession and how do their 
preferred practices align with ASHA's professional standards and existing policies?

According to Foss \& Waters (2007), as patterns emerge, questions may need to be refined. As such, the research questions above were slightly refined to guide the study.

\section{Justification for Research and Sampling}

Critical theorists argue that people often do not agree on meaning therefore research that defines terms clearly enough enables different people to agree sufficiently so they can get on with their acquisition of knowledge and being a certain kind of person (Fraenkel, Wallen \& Hyun, 2012). To properly apply this tenet within a qualitative design, a purposeful sample was obtained in which indepth interviews of groups and individuals were conducted. The interview questions were refined and semi-structured in order to gather meaningful and informative, yet natural responses. Since I am a SLP working in the public schools, I maintained a reflective journal to account for biases. Before I proceed with the sampling procedure, the next paragraphs will outline a detailed justification, based on context, for selecting the specific methods and approaches I used.

In the earlier stages of my proposal, I set out to write a dissertation that would solve the new problems that Rhode Island public school SLPs (including myself) encountered when a rule concerning the elimination of speech therapy services for nine-year-old students was passed in 2007. My research began with a review of the regulatory background and rationale for the nine-year rule. The Open Policy Act allows the public to view, comment, and record conversations in regulatory meetings and hearings. The documents pertaining to the nine-year rule 
are housed in the Office of the Secretary of State and include announcements of hearings and written commentary deadlines and meeting agendas (Szydlowski 2016). I found archived documents known as ERLIDs that track regulatory motions, some of which were needed because of clerical errors. While it was easy to access ERLIDs, recorded minutes of board meetings were not. However, I contacted Hannah Gallo, a Representative in Rhode Island General Assembly, and requested information regarding the passing of the rule $(\mathrm{H}$. Gallo, personal communication, December 15, 2015). Ms. Gallo replied with a copy of minutes that corresponded with the rule's passing in June of 2010 (see Appendix C). In those minutes, Deborah Gist made a motion to align state policy with federal policy and embedded in that alignment was special education eligibility criteria that included changes in speech services eligibility, otherwise known as the nine-year rule.

According to McGuinn (2015), legislation is often conducted in an expedited and chaotic manner to facilitate the implementation of political agendas. In 2016, I had access to a large survey on that rule. After conducting a pilot study, which included an analysis of SLPs' survey responses about that rule, I concluded that the process and the rule were unfair. I was not alone. The survey found that many Rhode Island SLPs had negative responses to it as well. As I defined the problem, I had to revisit the political and cultural events at the federal and state levels for a clearer understanding. Those legislative changes combined with the state's 2007 rule made SLPs' work very complicated. The findings were troublesome because despite opposition at public hearings from SLPs, their 
affiliated professional organizations, and the parents of the children impacted, the rule did go into effect.

As I proceeded with my study, I started to blame the general population of the state's SLPs — at that point, on the surface it seemed that they were not creating enough resistance to the rule or their work situation. With much discussion and guidance from my major professor, I realized that I needed to understand how oppression and power work and that it was compassion not blame that was necessary. From that point on, the study's focus became one of developing a clearer understanding of the situation, which led to the recognition that I needed to uncover the actual sources of the escalating problems we SLPs were facing as we tried to serve the student population and the profession of speech-language pathology.

I accomplished this through the data obtained from focus groupswhich included SLPs from across the state-and one-on-one interviews. As a qualitative researcher, I took steps to remain mindful of my role as interviewer and of my biases. However, because I was part of the system, I had embarked on an emotional journey. It did not take long to realize that my qualitative research necessitated that I adopt a critical perspective in order to avoid having my own feelings of injustice and the biases associated with them taint the analysis of the data.

Through reflective journaling I was able to unpack the complexities associated with tradition and history that appear so orderly and natural that they can go unnoticed and unchallenged (Gee, 2014). Due to the complex and dynamic nature of those conditions, I had to reflect and monitor my positioning and findings 
along the way. As a result of writing reflections, I became aware of my tendency to accuse SLPs rather than delving into other factors (Kleinman \& Copp, 1993). Since I was an insider, this journal was essential for acknowledging my thoughts/feelings and to discover any occasion in which I might have influenced others' knowledge, beliefs, or values.

\section{Steps before Data Collection}

Although there are other chapters in this study that go into greater detail about the state rule that caused problems, some explanation is necessary here for a better understanding of my choices of methodology. Late in 2007, the Rhode Island Board of Regents adopted new regulations that would limit and, in most cases, eliminate speech-language services for some nine-year-old students with disabilities. According to what SLPs called "the nine-year rule," SLPs were responsible for notifying parents that speech services would terminate on their child's ninth birthday. Only students with coexisting disabilities could retain services. In 2015, the American Speech, Hearing, and Language Association (ASHA) distributed a survey to only Rhode Island public school SLPs at the request of the Rhode Island Speech and Hearing Association (RISHA) in order to obtain their comments and responses to the nine-year rule. RISHA is a state professional organization run mostly by volunteers. They often consult with their national organization, ASHA, on professional matters, i.e., billing, ethics, and legalities. That survey yielded a $40 \%$ response rate with 140 of 337 SLPs responding. Also, $66 \%$ of those respondents wrote comments claiming the rule was unfair, 
discriminatory and arbitrary. The results of this survey informed my research questions and theoretical lens.

For the purposes of further analysis of the results, in 2016, I obtained permission from ASHA to use the results of the survey and then submitted a request to Rhode Island College's Institutional Review Board (RIC IRB) to use the survey to help inform future research. Once I received approval, I invited the executive board of RISHA, made up of volunteer SLPs, to review the results of the ASHA survey. The stated objective was to find meaningful categories for future study through the comments about the nine-year rule. In the first of three meetings, SLPs read 10 pages of comments and highlighted repeated words or phrases that were expected, unusual, and/or surprising (Creswell, 2014). The SLPs received a copy of the highlighted commentaries for further analysis.

\section{Pilot Summary}

There were two phases of the pilot study - the first resulted in preliminary choices for categories and the second confirmed those categories. A team of four SLPs and I met over the summer of 2016. The team was divided into two pairs and each SLP received colored highlighters, index cards, and envelopes. The color system provided some anonymity and a tracking system to ensure SLPs' perspectives were represented. The highlighted commentaries were extracted and organized under appropriate categories onto index cards. The first team of SLPs accounted for sub-themes with keeping a tally on the backside of the card. Similarly, the second pair of SLPs organized and labelled comments into categories, which were then compared with those of the first team. The categories 
were then compiled in an eleven-page matrix in which the highlighted comments from their first meeting were included. SLPs revisited that matrix in the second phase of the study. That final phase produced agreeability percentages indicating a prevalence of certain categories.

The SLPs had created forty-two cards, which initially fell into eight categories. Two of the four SLPs re-sorted the cards, making certain that all colors were represented. At this point, the group identified a certain number of categories, reducing the original number from eight to six, which were then included in the matrix. In the second phase of the study, that matrix was distributed to the whole group of SLPs, then half the group. They were instructed to read through the comments and decide which of the eight categories fit comments. The first group of SLPs had an acceptable reliability measure of $64 \%$ (Frankel, et al., 2012). Another rating measure was obtained with a second group of raters having $83 \%$ agreement between categories and comments. Of significance, the remaining $17 \%$ of comments and categories that were not agreed upon were consistently split with one of the categories, discriminatory (See Table 1). Of note when there are more than four categories being considered, $60 \%$ agreement is a reliable measure (Fraenkel et al., 2012). With that second rating, higher agreement between categories resulted in six categories and emotions and discrimination were ranked as prevalent. According to Miles and Huberman (1994), coding that agrees at least $80 \%$ of the time is good for qualitative reliability. 
Table 1. Pilot study results

\begin{tabular}{|l|l|l|l|l|l|l|l|l|c|}
\hline Categories & 1 & 2 & 3 & 4 & 5 & 6 & 1 vs. 3 & 1 vs. 5 & 1 v. 6 \\
\hline Percentage & 28 & 2 & 6 & 12 & 31 & 4 & 9 & 6 & 2 \\
\hline
\end{tabular}

* Categorical Index:

$1=$ Arbitrary/Discriminatory: Why target a 9-yr. old?

$2=$ Academic expectations preside over special education: Common Core is more important.

$3=$ Reactions to authority: People in power do what they want, they don't care about what we think.

4= Most Impacted, certain students: $I$ worry about students with stuttering issues, they will not get speech now.

5= Emotion: Defensive/ Offensive: We have so many numbers, it's hard. Or, in a way, this helps us to exit kids.

$6=$ Inadequate delivery of services for student impacted 9-yr rule: We can only do what we can do!

\section{Pilot Reflection}

These ratings prompted more questions for my investigation. First, the prevalent themes aligned with the theoretical frame, Pedagogy of Discomfort, which examined feelings and witnessing as potential sites of transformation (Boler, 1999). SLPs often encounter dilemmas in the wake of new regulations; however, they need to go beyond just bearing witness in order to accomplish satisfactory changes. Boler asserts discomfort is a pivotal step to agency (1999) and depending on the direction of that pivot, agency is either lost or gained. For example, SLPs emotionally 
articulated a rule had marginalized certain students in which they often felt powerless. Yet, some of their responses had legitimizing effects when they stated that rule ultimately offered SLPs reprieve from heavy workloads. That sort of pivotal decision-making mattered to their agentive potential. Second, discriminatory remarks were prevalent. While discrimination and unfairness were noted, authority, i.e., was not discreetly named as a concern. When power is neither visible nor contested, an analysis of themes of a discriminatory nature requires a more critical lens. Although the presence or absence of power is not usually considered to be an emotion, according to Boler (1999), a sense of powerlessness bears the qualities of emotions such as fear, anxiety, and denial. Therefore, Boler's theoretical views would be appropriate in responding to SLPs' concerns of discrimination and authority in their emotional testimonies. Since SLPs rated discrimination and emotion as prevalent yet neglected to see the role that power and authority were playing in their SLP practice, critical theory and critical discourse analysis was also appropriately applied. These categories informed the unit of analysis and research questions and facilitated an examination of SLPs' agency and identity.

The pilot outcome was shared with RISHA members, with three of the pilot participants present. The prevalent categories were named and discussed. Those present agreed that the findings were interesting and made them think more about power. We spoke about this issue at greater length. When we discussed discrimination, I asked them why they thought that the survey's results revealed agreeability on authority at only $6 \%$. One SLP simply stated, "We don't know who is in charge." Other questions surfaced about how this rule came to be and more 
importantly, why legal action had not been taken. One SLP stated that legal actions were pointless because the rule was not an actual regulation; hence, it was not legally binding. Another responded that it was financially driven-a money saver for the state since it removed many students from special education services at age nine.

From that reflective discussion and the results of the agreeability percentages, it was clear that many of the SLPs who had responded to the pilot survey were basing their beliefs about the nine-year rule on assumptions and general feelings rather than well-researched facts. They had a "sense" that the policy had already been formalized; hence they had few if any options for fighting the rule. These SLPs felt that they lacked any clear way to alter their situational dilemmas because they did not know how to successfully alter or eliminate the rule that they blamed for the problems.

\section{Research Design}

Having provided an overview of the naturalistic paradigm in which a critical and social perspective was justified, I will now outline the procedures used to collect, analyze, and transform data.

\section{Data Collection and Sampling}

Sampling. As a critical researcher, I needed to consider the context in which the study's SLPs had been situated in order to determine how their past and present experiences had contributed to their current observations and experiences. I also needed to consider work aspects for its potential influences, i.e., school location, school type (elementary and secondary), workload/caseload numbers (see Table 2). One very important factor to consider arose out of my own experience as a 
secondary level SLP. I needed to include the perspectives of SLPs who worked with secondary level students, since they were the ones who were actually confronting the results of the imposition of the nine-year rule on students with speech language problems.

Table 2. Work aspects: workload, level, and locale

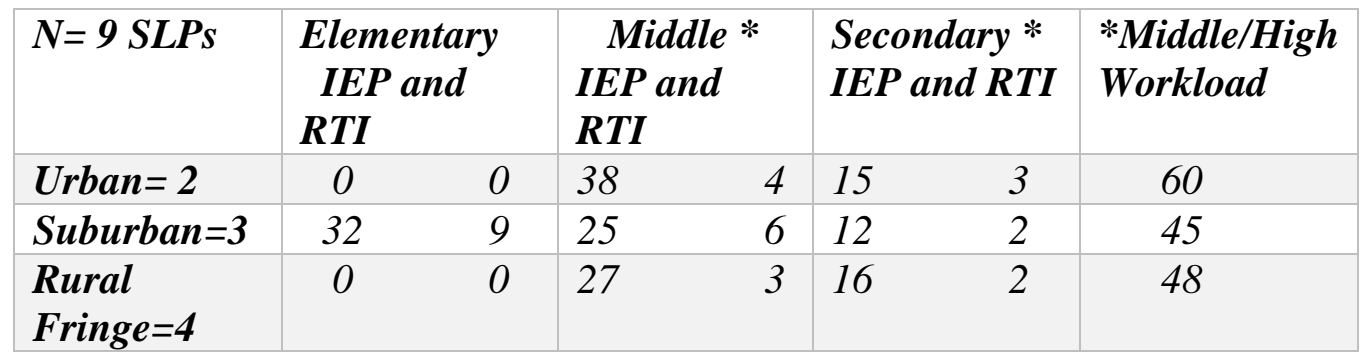

Based on these criteria, I attempted to obtain purposeful samples for focus groups - one with SLPs working at the secondary level and another with SLPs from across the state working at different levels (See Table 3). At first, I did not consider gender and ethnicity to be important sampling criteria since this profession is mostly comprised of white women. However, when my sample groups resulted in the presence of two male SLPs, I discovered that there were some differences between their perceptions and those of their female counterparts, particularly in the area of workplace practices. That observation was noted in my memos for a possible future study.

Table 3. SLP interview and focus group participants

\begin{tabular}{|l|c|c|c|l|l|l|}
\hline N=9 SLPs & Age & $\begin{array}{c}\text { Years } \\
\text { Working }\end{array}$ & $\begin{array}{c}\text { FG 1 } \\
\text { RISHA }\end{array}$ & $\begin{array}{l}\text { FG 2 } \\
\text { MS }\end{array}$ & Level & Region \\
\hline Eddie & 47 & 10 & $X$ & & K-12 & Urban \\
\hline Tim & 58 & 32 & $X$ & & Middle/High & Urban \\
\hline
\end{tabular}




\begin{tabular}{|l|c|c|c|c|c|l|}
\hline Toniann & 59 & 33 & & $X$ & Middle/High & $\begin{array}{l}\text { Rural } \\
\text { fringe }\end{array}$ \\
\hline Sandy & 47 & 21 & & $X$ & Middle/High & $\begin{array}{l}\text { Rural } \\
\text { fringe }\end{array}$ \\
\hline Katelynn & 25 & 1 & & $X$ & Middle/High & $\begin{array}{l}\text { Rural } \\
\text { fringe }\end{array}$ \\
\hline Ava & 43 & 16 & & $X$ & Middle/High & $\begin{array}{l}\text { Rural } \\
\text { fringe }\end{array}$ \\
\hline Sarah & 36 & 9 & $X$ & & $K-6$ & Urban \\
\hline Patrice & 46 & 21 & $X$ & & $K-6$ & $\begin{array}{l}\text { Rural } \\
\text { fringe }\end{array}$ \\
\hline Kate & 36 & 13 & $X$ & & $K-6$ & Suburban \\
\hline
\end{tabular}

Prior to initiating the sampling procedures, I first had to obtain IRB approval at RIC. In order to obtain elementary and secondary SLPs with diverse ages, experiences, and work settings, I was permitted to access the member listserv from RISHA (see Appendix E for procedures and approval). Fowler (2014) states that focus groups should have at least four members. However, due to a low level of interest, I had to be proactive in gathering enough participants from across the state for two focus groups. As such, I spoke at RISHA events that offered CEUs for attendance and where I had raffles for interested members, which did enable me to form two groups. Considering the sample was convenient, I was fortunate to assemble a diverse group with a $21 \%$ representation of men, which is considerably higher than the national norm of $4.5 \%$.

In addition to these two groups, I also conducted follow-up face-to-face interviews to address certain topics that had surfaced in the focus groups that needed more in-depth discussion.

There were several steps involved in the initial process of securing participants. First, I assured them that their identity and data would be secured. 
Next, I sent consent forms to participants informing them of the study's intent and the data instrumentation and collection procedures, which included an electronic survey and two phases of interviewing: focus group and individual. Participants knew they could refuse or exit the study should they encounter any issues with the study. I informed them that their identities in the interviews would be protected by the use of pseudonyms and that the electronic surveys would be configured to be anonymous. In addition to their identities being protected, I also informed them that the audio-recordings and collected data would be secured. The candidates were told that the survey included the collection of demographic information about them but that the server identity would be configured for anonymity.

Because of the transparency of the information process in the consent forms, I am confident that the SLPs were authentic in their responses.

\section{Data Sources}

\section{Regulation Review}

Using the Rhode Island Department of Education website, I obtained documents archived in the Secretary of State Office of Rhode Island. I searched regulatory documents titled ERLIDs which were organized by organization (i.e. Board of Education), title, and date. I sorted through filings surrounding special education criteria dating back to 2007-2008 because that was when school districts implemented the nine-year rule. The ERLIDs served specific functions, i.e. revisions, repeals, and amendments, many of which were necessary to correct clerical and technical errors. In addition to the state's acknowledgement of errors, 
I recorded my observations surrounding rushed timelines and announcements that appeared out of order (See Appendix F).

\section{Semi-structured Interviews}

I conducted focus group interviews and face-to-face interviews using Carspecken's (2013) protocol of semi-structured interviews which calls for questions that naturally emerge. To maintain naturalness and openness, I began the interviews by asking three to four open-ended questions and then implicitly led the conversation, adding questions that became more explicit later in the discussion (see Appendix $\mathrm{G}$ for focus group and interview questions).

Focus groups. I used the Google poll app for scheduling focus group slots and subsequent interviews. All interviews were at least an hour long. Focus group sessions were conducted at a public library that was not near to the participants' school districts. I conducted the focus groups in private library rooms in order to facilitate a comfortable speaking setting that would allow for uninhibited conversation. I used two audio-recording devices to ensure the capture of all conversations on both sides of the table. I also took notes during the sessions. Since the questions were semi-structured, I was able to take notes while participating.

Face-to-face interviews. Participants chose second interview sites for convenience and comfort purposes. Before scheduling the face-to-face interviews, the focus group audiotapes were manually transcribed. This was necessary in order to discover the prevalent and surprising findings that lent themselves to further discussion. The transcripts from both recording devices were cross-referenced for commonalities, differences, and accuracy. I also used a transcription service to 
validate information. The transcripts were first analyzed using coding techniques and then critical discourse analysis.

\section{Data Analysis}

There was a multi-step process for transforming and analyzing data. Prior to this study, I conducted an IRB-approved study in which the prevalent categories were identified. Those categories informed the questions that guided this study. Next, open coding was conducted whereby patterns were identified and integrated. I used Foss \& Waters' (2007) recommendations to help answer research questions and explain findings. Finally, since language was critical to the SLPs' formation of their identities, their discourses were analyzed.

\section{Coding and Transformation}

I uploaded manually transcribed interviews to SATURATE. This digital application facilitated the comparison of data across sources. In this way, I was more easily able to categorize and code the SLPs' responses. Within that application, I was able to log analytic memos and constantly view and compare data according to codes and sources. By way of this review, three-hundred and sixtynine chunks of information were initially organized and coded under sixteen themes. To focus the analysis and make codes more manageable and explainable, I referenced linguistic and metaphoric representations, which is a process applied in rhetorical analysis.

To transform and explain data, I envisioned patterns that emerged from codes and themes using techniques described by Foss and Waters (2007). These techniques are usually applied in rhetoric analysis. Following Foss and Waters 
(2007), I selected visual and linguistic representations after rearranging codes; as a result, I was able to better define themes and conceive of an explanatory schematic that included codes and corresponding themes. Three major categories resulted: systems, complications, and dilemmas. Those categories accounted for the sixteen themes, which after Foss and Water's techniques, were appropriately rearranged and renamed.

To proceed, I extracted the sixteen themes from SATURATE and labelled sixteen envelopes of three different colors with those themes. The colors represented research questions. Printouts of texts from SATURATE were inserted into color-coded envelopes that answered research questions. The contents of the envelopes contained a minimum of 10 to a maximum of 45 chunks of information that represented interviewees' comments. Foss and Waters (2016) recommends that envelopes be intentionally mixed and subsequently rearranged to form an image. In that process, I noticed that many of the envelopes did not discretely answer the research question. To manage data integration, I decided to duplicate themes onto colored index cards of varying sizes. By doing that, I was able to visually represent and quantify data to answer the questions.

Foss and Waters (2016) additionally suggests using visual metaphors to integrate data points. Therefore, I conceived of symbols to match findings-for example the Eye of Horus, a symbol for safe journey, helped to illustrate the manner in which SLPs' navigated dilemmas. At the core of their navigation, I visualized SLPs' emotions as a compass needle, guiding their direction. Eventually, I devised an explanation that helped define and answer research questions and confirm 
codes (see the operational definitions for the identified themes and explanations in chapters 4 and 5).

\section{Critical Discourse Analysis}

I focused on seeing how one aspect of critical discourse analysis, building tasks, were visible in data. To do so, I applied Gee's building tasks to the SLPs' transcribed interviews for understanding SLPs' ways of doing, saying and interacting. By analyzing SLPs' social and linguistic discourse, I reveal the devices they used to build significance, get recognition, enact relationships, make meaning, privilege dominant structures, and ultimately, shape identities (Gee, 2005). Guided by these aspects, I identified and explained their part in systems, complications, and navigation of dilemmas.

I used Gee’s (2011) language-in-use as a tool to ascertain how language was functioning and for what purposes. Foss \& Waters (2016), like critical theorists, claim that metaphors and clichés share similar functions. Metaphors are age-old representations and have withstood the test of time; this familiarity and tradition was a colorful and effective way for SLPs to access and convey words with which others could connect and recognize. According to Gee (2011), intertextuality is an effective communicative devise that includes the application of familiar text in another context; and in doing so, certain messages are signaled. Likewise, interdiscursivity is a mediating element whereby discourse is rearticulated using stable combinations of discourse, genres, voice, and styles so that one can understand at a more concrete level (Fairclough, 1992)). Graham (2016) adds intertextuality contributes to and enhances the constitutive properties 
of discourse whereby the recognizable aspect of that discourse acts to legitimize ( $\mathrm{p}$. 16). For example, SLPs' reestablished the identity of professional as one who follows the law and in doing so they legitimized the rule.

Gee (2011) refers to deixis or shifter words that tie context to what one already knows; and when used, those words help to locate one's positionality. These words include pronouns, articles, i.e. "the", and conjunctions that function to stress location and social ranking. Fairclough's conception of voice is applied similarly and consists of the following grammar structures: pronouns that position one in relation to another, active and passive voice for distinguishing agency or lack thereof, and verb types, i.e. linking and active that show positionality and agency (Rogers, 2008). When SLPs use these devices in their interactions and socialization, they are locating themselves in response to dominant structures.

According to Gee (1999; 2008), value is assigned through being socially recognizable and helps one adopt or recruit, and in certain contexts, resist identities. In the focus groups, SLPs' recognitions were filtered through perspectives based on values and beliefs; participants' actions and practice, or the way they talk, dress and act in their interactions; their application of knowledge; and finally, their experiences and background. In this study, participants were open about their feelings and often used humor and metaphors to voice their opinions. Of note, Fairclough (1992) classifies humor as a genre to which language links one to a particular social activity (as cited in Rogers, 2011, p.150). Beneath these social and literary devices are emotions and feelings, which according to Boler (1999) have transformative potential for professional identities. 


\section{Validity and Reliability}

In any research, limitations may affect study findings. Qualitative research enhances the validity of the findings by enhancing trustworthiness through dependability, credibility, confirmability, and transferability (Guba \& Lincoln, 1998). Dependability and credibility have been addressed in data collection procedures and analysis sections, i.e., initiatives for protecting identity/data and analysis methods. Validity is achieved with CDA in four ways: convergence of data, agreement of data, coverage of data, and attention to linguistic/grammar details (Gee, 2014, p.196). In the consent letter, I detailed other steps to establish credibility, dependability, and trustworthiness (See Appendix E). I achieved confirmability by comparing information across data points and by allowing participants to check transcripts. In addition to member checking (Patton, 2002), I conferred with a critical friend who is also a researcher about the findings and my thoughts on the data in order to continue to preserve as much of an objective view as possible.

Finally, reflective journaling added to validity as I accounted for subjectivities. Critical theory is value-mediated and requires an understanding of the conditions rooted in culture, politics, and economics (Guba \& Lincoln, 1998). With that in mind, I recorded my findings and reflected on them. The results of this process often necessitated follow-up conversations with my major professor. The process of journaling and conferencing raised my consciousness, which was a positive contribution to the final product. 


\section{Limitations}

Transferability is limited in that the samples in this study were restricted to SLPs in a suburban school and to RISHA members. Although a convenient sample of RISHA SLPs was recruited, there was no guarantee that their work and background would be quantitatively different. This was clear from national statistics: the gender percentage of SLPs working in public schools across the nation is $95.5 \%$ women (Edgar \& Rosa-Lugo, 2007). Knowing this in advance, I used an online survey as well as notes from the study to describe as richly as possible the experiences that unfolded. With that in mind, researchers can apply findings as appropriate.

\section{Summary}

Chapter 3 outlined the philosophical grounding for this study's design, the methods of analysis, and the procedures selected. The naturalistic paradigm and critical theory standpoint were described along with the rationale for the qualitative methodology. This chapter also provided the rationale and justification for this study's research design. Since critical theorists revisit the histories of the participants as a way to understand the findings, a decision to include the results of a pilot study was essential to understand the study's SLPs' responses to questions about policies. As such, the outcomes of that pilot were included in this chapter. Since background and experience are part of the SLP's histories, I interviewed SLPs about past and present events that factored into their identity formation. Critical theorists acknowledge the power of language and socialization in the transmission of value and worth; therefore, a focus group was essential to 
understanding the SLPs' interactions and their use of linguistic devices and practices. The progress of this study included an examination of their discourse in which subsequent transformation revealed not only their challenges but also their navigation of systems.

Chapter 4 presents the findings of this study, describing the systems and corresponding complications that led to the SLPs' dilemmas. Chapter 5 delves into a navigational system that SLPs improvised during moments of confusion, frustration, and conflict. Chapter 6 discusses the implications of this study's findings and makes recommendations for future research and practice. 


\section{CHAPTER 4: RESULTS}

\section{Introduction}

This study uses a critical lens to examine the relationship between power and discourse for Rhode Island public school based SLP's as they navigate conflicting federal and state policies that impact their work. The findings of this study are organized into two chapters, four and five, where SLPs' discourse is analyzed as it relates to three guiding research questions: 1) What challenges and tensions do SLPs experience in delivering speech services for students targeted by the nine-year rule in relation to RTI, and how do they respond? and 2) What experiences and values do SLPs perceive as significant in the formation of their professional identities? Research Question Three (How do SLPs integrate their values, ethics, professional experience and background/education with the norms of the profession, and how do their preferred practices align with ASHA's professional standards and existing policies?) will be explored and analyzed in Chapter 5.

In this chapter, I operationalize definitions of systems and complications (See Table 4) then begin with an analysis of the complications associated with the first two systems (See Table 5). I describe two key professional organizations for Rhode Island SLPs: the American Speech and Hearing Association (ASHA) and the Rhode Island Speech-Hearing and Language Association (RISHA), and the relationship with federal and state policies; both of which are systems that complicate SLP work. 
Table 4. Definition of systems and complications

\begin{tabular}{|l|l|}
\hline \multirow{5}{*}{ Systems } & $\begin{array}{l}\text { Powerful structures that influence acts and minds of SLP } \\
\text { members through authorities that operate ambiguously. In } \\
\text { that manner, authorities control flow of knowledge and } \\
\text { information benefitting an agenda that does not benefit } \\
\text { students'needs or the SLP profession/practice. }\end{array}$ \\
\hline Complications & $\begin{array}{l}\text { Work site problems that result from a misalignment and/or } \\
\text { intersection of systems. When SLPs inhabit systems, they } \\
\text { are working under conflicting agendas that do not serve } \\
\text { their students'need and meet expectations of their } \\
\text { professional mission. }\end{array}$ \\
\hline
\end{tabular}

What Gramsci (1971) calls "hegemony" are systems that integrate in laws, norms, habits, and even consensus. That intersection appears natural; hence making it difficult to notice and understand (as cited in Boler, 1999). As systems intersect, they controlled the trajectory of the SLP career and created complications and disorienting moments. SLPs navigation of these disorienting moments had professional implications. In this study, SLPs noticed complications; yet, how they acted in those dilemmas depended on how they were situated and treated. SLPs are institutionally bound in that they existed in different contexts over time, i.e. training and interning in the university institution and working within the education institution with a variety of people, places, protocols, and programs. Chapter 5 examines SLPs' discourse across systems as they interact with other participants at various institutions and explores how SLPs negotiate relations and manage their identities and agency.

\section{A Myriad of Factors Concerning SLP Identity and Agency}

Data for this study revealed a myriad of factors both inside and outside the SLP discipline that present challenges and complications for SLPs when they 
advocate for and serve students with speech sound disorders (SSD). The complications stem from a necessary interface with multiple and often conflicting systems and solutions, which created entangled and convoluted situations for SLPs. For example, when serving particular students in complex systems, an SLP must adapt protocols much like a doctor must adapt the medical protocols necessary for one particular condition when treating a patient with generally compromised immunity. SLPs acknowledge that multiple systems are at work; however, they often devise explanations and solutions that perpetuated rather than addressed the problem. While SLPs could effortlessly identify and describe their working complications and their symptoms, i.e. large and complex cases, scheduling conflicts, inadequate resources, etc., it was harder for them to diagnose, understand, describe, or respond to the underlying system behind these complications. In these situations, SLPs' agency took on different forms such as creating explanatory and accusatory discursive practices. However, those initiatives may have done more to entrench the complications than to resolve them. As a result, those devices have implications for the SLP career, which are summarized in the concluding chapter.

\section{Systems}

The data secured through the transcribed discussions of two speechlanguage pathologist focus groups as well as follow-up individual interviews indicated that some SLPs seemed unsure or even unaware that they were working under various systems. The systems--each powerful and purposeful in its own right--are structures that are organized, dynamic and ambiguous in nature. In fact, 
that ambiguity results from circumstances and conditions for which there are no set responses. As such, SLPs may improvise while others may not recognize the source of complications (Holland et al., 1998; Nygreen, 2013). Consequently, when one attempts to attend to the ambiguities, the systems happen naturally and go undisturbed (Fitzgerald \& Callard, 2015, Bolman \& Deal, 2013). As I analyzed SLPs' words, I was able to categorize and operationalize three primary systems: 1) Professional Organizations, 2) Federal and Local Policies, and 3) Educational Institutions. Finally, as systems entangled, SLPs attended to those ambiguities which were often taken-for-granted. See Table 2 below for a brief summary of the systems in terms of purpose, nature of ambiguities, and the complications.

Table 5. System chart

\begin{tabular}{|c|c|c|c|}
\hline Name & Purpose & Ambiguity & Complication \\
\hline $\begin{array}{l}\text { ASHA and } \\
R I S H A\end{array}$ & $\begin{array}{l}\text { Profit and Non- } \\
\text { Profit professional } \\
\text { organizations } \\
\text { whose missions } \\
\text { include advocating } \\
\text { for the SLP } \\
\text { profession and the } \\
\text { clients they serve. }\end{array}$ & $\begin{array}{l}\text { Two organizations } \\
\text { that perform under } \\
\text { different structures, } \\
\text { one is responsive to } \\
\text { all states while } \\
\text { RISHA is attuned to } \\
\text { state only issues. }\end{array}$ & $\begin{array}{l}\text { The multiple affiliations and } \\
\text { roles of ASHA are widespread } \\
\text { across the states while RISHA } \\
\text { has minimal resources run by } \\
\text { non-volunteers. Hence RISHA is } \\
\text { reliant on ASHA which impacts } \\
\text { their effectiveness when dealing } \\
\text { w/state issues that impact SLPS' } \\
\text { practices. }\end{array}$ \\
\hline
\end{tabular}




\begin{tabular}{|c|c|c|c|}
\hline Policy & $\begin{array}{l}\text { Federal: NCLB, } \\
\text { IDEA, ADA, RTI, } \\
\text { ESSA and State: } \\
\text { nine-year rule } \\
\text { makes legislation }\end{array}$ & $\begin{array}{l}\text { Legislation, policy, } \\
\text { and regulations in } \\
\text { which views are } \\
\text { conflicting and } \\
\text { resources vary that } \\
\text { result in struggles } \\
\text { for power. Also, } \\
\text { constituents } \\
\text { influence elected } \\
\text { officials. }\end{array}$ & $\begin{array}{l}\text { Policy responsiveness depends } \\
\text { on failures of systems, a } \\
\text { never-ending problem. } \\
\text { or } \\
\text { where power is most } \\
\text { concentrated on a } \\
\text { neoliberal focus consisting } \\
\text { on cost reduction, production } \\
\text { accountability. } \\
\text { As a result, allocated duties } \\
\text { are often } \\
\text { driven by numbers. } \\
\text { All of which have shaped } \\
\text { culture and education. }\end{array}$ \\
\hline $\begin{array}{l}\text { Education } \\
\text { Institution }\end{array}$ & $\begin{array}{l}\text { Social architecture } \\
\text { in that people } \\
\text { (their interaction } \\
\text { and perceptions) } \\
\text { alongside } \\
\text { protocols and } \\
\text { procedures at } \\
\text { specific places are } \\
\text { part of an } \\
\text { institution } \\
\text { assembly }\end{array}$ & $\begin{array}{l}\text { That assembly, } \\
\text { responsibilities are } \\
\text { allocated with focus } \\
\text { on educating and } \\
\text { helping students. If } \\
\text { part of the assembly } \\
\text { deviated or } \\
\text { malfunctions, the } \\
\text { entire system is } \\
\text { impacted }\end{array}$ & $\begin{array}{l}\text { Perceptions and values } \\
\text { become an issue to contend } \\
\text { with which impacts } \\
\text { working roles. } \\
\text { The have and have not situation } \\
\text { results impacting people, } \\
\text { protocols, and procedures. }\end{array}$ \\
\hline $\begin{array}{l}\text { System } \\
\text { Entanglement }\end{array}$ & $\begin{array}{l}\text { The overlapping of } \\
\text { the three primary } \\
\text { systems in which } \\
\text { SLPs respond to } \\
\text { integrated norms, } \\
\text { rules, habits, and } \\
\text { even consensus }\end{array}$ & $\begin{array}{l}\text { Naturally happens } \\
\text { when participants } \\
\text { attempt to contend } \\
\text { with complications. }\end{array}$ & $\begin{array}{l}\text { The critics and supporters } \\
\text { (SLPS/teachers/parents) do not } \\
\text { see or do not want to see the } \\
\text { effect of systems. Consequently, } \\
\text { complications stemming from } \\
\text { misalignment create dilemmas } \\
\text { which differ for SLPs. }\end{array}$ \\
\hline
\end{tabular}

\section{Implication of Systems}

In this chapter, I explain how the first two systems impact SLPs'

work. SLPs experience the influences of ASHA while in college and training

then later alongside RISHA in their work in Rhode Island public schools. They 
strive to adhere to the organizations' mission of excellence in practice and advocacy for clients and the profession. However, the multiple and widespread roles of the national organization alongside a small-membership state organization make this mission a challenge. Hence, SLPs' responses and perceptions to these organizations were somewhat negative. The second system, federal and state education policies, also have a huge impact on SLP work and often conflict with the first system, particularly because of the changing face of education for special needs children over the last 20 years. Chapter five explores how the social architecture of educational institutions constrained SLPs work and factored into the formation of SLP identity and their agency. The fourth system, which I have labeled System Entanglements, is an intersection of all systems and had the most perplexing complications for SLPs. The manner by which SLPs navigated this system had implications for the profession and will be discussed in chapter 6.

\section{Definitions of Terms: Systems’ Complications and Dilemmas}

In this section, I define how I am revisiting systems in terms of their complications and dilemmas, as those terms are key to this research. In any organized structure, problems exist when the assumptions are misaligned, mishandled, and misappropriated; and with system entanglements, sorting out those distinctions mattered in resolution (Bolman \& Deal, 2013, Bell, 2014). Depending on the structure, or in this case, the systems, Bolman and Deal (2013) recommend tools for correcting problems; however, they do not address entangled issues. In this study, SLPs attempt correction without knowing the systems; as 
such, their agentive capabilities varied. In some cases, it appeared that while some SLPs understood those systems, they were hesitant and unsure of their facts. On the surface, the apparent intention of these systems was that of helping, supporting, and advocating for them. Most of the SLPs ably described the complications and frustrating dilemmas but they were unsure of the causes and ways to correct the problems.

At different points in their working careers, SLPs are subject to certain interfaces with systems that challenge their professional knowledge, beliefs, and values, as well as the many aspects of their work that they enjoy. For the participants of this study, when these systems interfaced, they took on other characteristics which made solutions to dilemmas more challenging.

\section{Complications}

In some instances, complications that occur in an SLP's professional practice happen when the SLP's knowledge from training and previous work experiences conflict with policies. This happens naturally since a system like a university is where SLPs have learned their trade and under ASHA, another system, is how SLPs attained certification. Ideally, SLPs learn to trust and endorse the protocols set forth in both of these systems. However, my data demonstrate that some SLPs become disoriented when these systems intersect or conflict. For example, the knowledge they acquired at the university level does not necessarily align with ASHA's expectations for scope of practice. SLPs learned from the university system and became credentialed through ASHA, so it follows that when those systems' expectations conflict, SLPs encounter serious 
dilemmas when attempting to determine appropriate services for specific students.

A major reason for these complications as it relates to ASHA is the fact that ASHA interfaces with multiple systems across the nation; i.e. universities, state regulatory agencies, and credentialing services. When ASHA overlaps with or is distracted by its other functions, it can unintentionally disturb the work of SLPs. Much like a person coping with compromised immunity, the interface of entangled systems often clouds the origin of the problem, thereby making the solutions for the resulting complications confusing and complicated. For example, SLPs may be confused on how to remedy the expectations of serving students across different systems, because ASHA's scope of practice includes literacy services, while under IDEA revisions, measures of oral language go unmonitored. In fact, this entanglement can constrain the mission of SLPs. Functioning optimally in an entangled system requires an alternative approach. The intended function between systems vary, i.e. one is regulating while another is promulgating. Consequently, the systems, especially the ones that interface, get muddled. According to Gee (2001), hidden systems are difficult to contest; I propose that entangled ones are as well. Either way, this study requires a look that is both critical and analytical.

\section{Dilemma}

Within the context of this study, the dilemma is the space in which SLPs discuss, negotiate, and sometimes solve the complications that are rooted in 
systems. The complications, i.e., large caseloads, parental concerns, complex cases, eligibility decisions, and logistical conflicts, exist in relation to systems. They become more difficult to contend with when dynamic systems, i.e., policies, build on each other or on in conflict with each other. They become entangled with the expectations and standards set forth by multiple systems, i.e. ASHA and the school institutions.

In this study, SLPs appeared to navigate their professional roles using the following, and occasionally conflicting, orientations: 1) doing what is expected combined with 2) doing what they know and 3) doing what is permitted or sanctioned. As they attempt to navigate dilemmas, they describe disorienting and confusing moments with emotional overtones. They define their ongoing understanding of what is expected within their scope of practice by what they learned in college and over time alongside what their workplaces provide or allow them to do.

\section{The Systems and Corresponding Complications}

The first system includes professional organizations at the national and state level. The American Speech-Hearing and Language Association (ASHA) and Rhode Island Speech-Hearing Language Association (RISHA) are organizations that assist SLPs in serving clients with unique needs. However, the manner by which they operate have created complications and dilemmas for SLPs which are described and analyzed in the following section. 
The second system covers federal and state policy which together brought on another set of complications. The complications were rooted in the neoliberal agenda of accountability, productivity, and cost reduction initiatives. Over time, policies overlapped and had blurred roles between special and general educators which created confusion for SLPs. Also, the focus on productivity and cost reduction created challenges for SLPs in how they in managed workload and determined eligibility of services for students. In Table 6 below, three complications per system are outlined and will be described in this chapter.

Table 6. Examples of systems and complications

\begin{tabular}{|l|l|l|}
\hline \multicolumn{1}{|c|}{ Systems } & \multicolumn{1}{|c|}{ ASHA and RISHA } & Policy \\
\hline Complication 1. & Multiple conflicting roles & Overlapping \\
\hline Complication 2. & Misaligned scope and mission & Numbers drive policy \\
\hline Complication 3. & ASHA thrives, RISHA exists & Policy fails to monitor \\
\hline
\end{tabular}

\section{ASHA and RISHA}

\section{ASHA's Multiple and Conflicting Roles are Disorienting}

The American Speech and Hearing Association (ASHA) is a large professional organization that credentials and guides speech-language pathologists (SLPs). In this study, it is categorized as a system because of ASHA's work with and influences on SLPs and the Rhode Island Speech-Hearing 
and Language Association (RISHA). ASHA certification requirements include graduation from an accredited master's program followed by a national board examination and the completion of a nine-month internship. Credentialed SLPs who want to continue to practice must earn 30 hours of continuing education units (CEUs) over a three-year interval, which SLPs pay for and ASHA monitors. ASHA charges an annual membership fee of $\$ 255$, part of which covers the management of their CEU registry. Since SLPs maintain credentials through ASHA, it is clear that ASHA has some control over SLPs' livelihoods and career trajectories.

Many interviewees believed that while ASHA was good at some of its functions, it was not doing enough to support SLPs in their profession and mission. On the positive side, the majority of SLPs thought that ASHA was an effective organization in terms of credentialing and providing research-based information. Eddie stated, "They are really good at the gatekeeping stuff." Although Sandy also valued the gatekeeping function of ASHA, she found the organization lacking in most other areas, declaring that "other than that licensing piece, I don't refer to them." These SLPs felt that there were important situations in which ASHA should have been more authoritatively supportive of work-related issues that they faced - for instance in fighting the nine-year rule, which they believed indiscriminately had to discharge nine-year-old students who had only been receiving speech services. Responding to a question about ASHA's part in 
the debate about the nine-year rule, Sandy said outright, "I don't feel like they supported us."

Ava addressed another problematic issue concerning ASHA. She perseverated on the costliness of ASHA, saying "you had to pay for that; you had to pay for-and again on that..." Given the fees, most SLPs stated that they expected more from ASHA, to which Toniann jokingly responded, "We pay them to keep quiet."

According to Foss \& Waters, rhetorical questioning and irony are useful in getting the attention of others (2007). For instance, in terms of SLPs' responses to Toniann's comment, they listened and laughed. Her humor and irony were effective in making the case that ASHA could be more supportive. She insinuated, through facetiousness, that ASHA was not vocal enough in addressing complications, in this case a state policy that conflicted with national norms and standards of excellence and advocacy. Although she found ASHA negligent, she implicated SLPs contributed to the problem by paying into the system.

ASHA's oversight is even broader than the aforementioned examples. It also plays a major role in both the education and career development of speechlanguage pathologists. In college, student membership in ASHA is encouraged with incentives such as reduced member fees, grant and research opportunities, and scholarships. Following graduation, ASHA offers resources such as practice tests for Praxis exams to which ASHA is also a contributing advisor. Once a SLP passes the exam, she or he embarks on a nine-month fellowship, which includes 
ASHA supervision and specified clinical hour requirements. ASHA also oversees a mandated and ongoing continuing education program in which SLPs are expected to accrue throughout their professional working years. Also, ASHA sits on boards that approve accreditation of Communicative Disorders Programs at many universities.

Given the presence of ASHA at all stages of an individual's choice to practice the profession of speech and language pathology, it would be fair to say that ASHA controls the trajectory of an SLP's career. Therefore, upon receiving accreditation, SLPs tend to be mindful of the organization's expectations for SLPs' scope of practice and how it affects them. SLPs discussed ASHA's code of ethics and mission that prescribed the standards they were expected and even obligated to meet, but they were also quick to point out their struggles to do so. For example, when determining services for so many students with different needs, Susie said, "Well, how could you say no to this boy?-like he could still use some help. But if I'm saying yes to you [ASHA], then I'm really saying no to you [the student]." Susie feels that she has to answer one way or another, that there is no in between and that is frustrating as Susie, by nature, needs definitive responses for answering which students she should treat that fall within the scope of practice and adhere to a mission of advocacy.

ASHA, like other power structures, operates broadly and ambiguously and, in doing so, creates ethical and professional paradoxes. Ava further illustrated this kind of dilemma that ASHA and its own professional creed creates 
for SLPs in these kinds of situations when she explained, "You know...ASHA has the provision of...the ethics piece, where you shouldn't do anything that you don't have the expertise in, or you don't feel as though you have the expertise in." As such, ASHA gives conflicting messages: first, treat all patients and do it well and, second, don't do it if you are not comfortable. Clearly, SLPs refer to ASHA's mission and code of ethics concerning issues in their practices, but they come away feeling confused and uncomfortable.

One clear role of ASHA is, as the SLP credentialing agency, it determines what SLPs need to learn and even how they learn it. For instance, ASHA creates the national Praxis exam and sits on the board that approves all SLP communication science programs. Overall, ASHA's responsibilities seemed to be operating in an executive capacity when it determines who gets credentials and what universities get accredited. Eddie credited ASHA in this executive role and thought that it was effective at controlling a standard of practice: "It's important to have a powerful and recognizable gatekeeper." In terms of its effectiveness in other issues, Eddie referenced hearsay when he said, "People_-I read, you know, in other places - say that they are an effective organization, that they are doing a lot." He indicated ASHA could be more capable when he stated, "With that money.... is it providing high quality professional development? No, it's not. It's not that, obviously." Eddie did not have an answer, but with all "that money" he hinted ASHA could be doing more than providing professional development 
(PD). While ASHA provides PD, Eddie recognizes ASHA has other functions and indeed money serves other purposes.

Unlike Eddie, Sandy's and Ava's comments about ASHA were on a personal level. Of note, Ava and Sandy are veteran SLPs with over 35 years of experience between them, while Eddie had only worked in Rhode Island for three years. This may in part account for some of the differences in their narratives. Sandy worked at the elementary level and because of that she had firsthand experience with the nine-year rule since it directly affected her ongoing client caseload. As a result of her experience at the public hearing on the nine-year rule, Sandy said, "We went through this whole nine-year rule protest, and I feel like we weren't supported; I felt like we were dropped," later adding, "I don't refer to ASHA, and I think that's sad." Sandy metaphorically conveyed this sense of abandonment with her use of the word "dropped," an active and emotive verb. While her first comment legitimized her actions against ASHA, the second comment conveyed her mistrust for an institution that should be supporting students which to her was sad. Indeed, Sandy was sad; however, it is uncertain if that sadness was only directed at ASHA; perhaps SLPs not using ASHA for professional resources was sad too.

Ava, a single-working mother, had worked part-time at a nursing home while working full-time at a middle school and a high school. She could not afford to lose her SLP license, which nearly happened when she missed the ASHA renewal deadline. Since Ava dealt with economic and personal pressures, 
the amount of money ASHA charged her for the renewal was of primary concern, and her comments gave voice to her dissatisfaction. "I feel like it's a lot of money and time. For me personally, I don't know what they've done...other than make me jump through hoops to get my license." Ava's comment was conveyed with a cliche, an effective literary device that communicates powerful images.

Ava, a veteran SLP, thought ASHA acted unfairly and unreasonably towards her when she had to be re-credentialed according to the same criteria as new graduates because she was late in her renewal application. She resented the resulting registration fees $(\$ 255.00)$, the Praxis Core examination cost (3 subtests at $\$ 900.00$ each), and the probationary period that prevented her from receiving her per diem earnings. Ava discussed the effects of ASHA's demands in terms of lost revenue. "I had to get reinstated because I couldn't see clients." As a result, it took a personal toll. "I was stressed.... and ashamed at the same time." After a pause, Ava sighed, an expression of both her exhaustion and her sense of shame at acknowledging her part in letting her license lapse. With that admission of shame, her frustration with ASHA seemed to recede. "I mean I understand that ASHA does a lot for our profession." Ava seemed to reconcile with ASHA and had completed the certification processes, i.e. re-took and paid for the praxis exam, provided proof of continuing education, and paid the application fee. While the process was time consuming and expensive, she acknowledged her responsibility. Although hesitant, she had accepted ASHA's position and complied with their protocols which resulted in her accreditation. 
In her interview, she shared that professionally and personally she struggled with her conflicting sentiments of resentment and shame regarding ASHA's diligence and her negligence in maintaining the necessary credentials, which resulted in the lapse of her professional license to practice and therefore her ability to earn an income. Finally, after a pause, she succumbed to a form of reconciliation when she stated that ASHA had done a lot for the profession. In spite of what she said, that pause suggested that she may not have been totally convinced about that belief and therefore not as vested in ASHA. Ava's lengthy pause signaled she was unsure of what she should say which speaks to her dilemmas where her acts could be perceived unfavorably. According to Gee (2014), what is not said is as meaningful as what is said.

From the data above, it is clear that while Eddie and Toniann were indifferent towards ASHA, Ava and Sandy were more upset with the organization. Eddie simply thought that ASHA was better at one function than another. He did not offer explanations or reprimands, just questions. Toniann reacted more like a disgruntled customer dissatisfied with a costly product, i.e. advocacy, that was not performing as it should. On the other hand, Sandy felt let down and abandoned; as a result, she had decided that she had no use for ASHA. Ava also felt let down, harboring resentment towards ASHA for requiring her to go through an entire re-credentialing process after years of practice. While able to recognize and describe areas of great discontent with ASHA, they endured complications and dilemmas because they do not see themselves as having any 
power against ASHA. Perhaps, Eddie's recommendation for increasing regulatory knowledge and SLP participation on a social media forum would gain ASHA's attention and result in changes.

\section{A Mismatch between Scope of Practice and Expectations}

SLPs claimed that ASHA had greatly extended their scope of practice which means they are expected to treat disorders of various etiologies, i.e. medical, congenital, developmental, and/or acquired. More recently, declining scores on reading and writing proficiency for students in middle and high school resulted in ASHA's recommendation that SLPs participate in adolescent literacy interventions (ASHA, 2011, 2015). ASHA's response to those proficiency scores created an expectation in which SLPs had to serve more students with diverse needs. In the next chapter, I will elaborate on a state regulation that complicated that expectation. In the focus group discussions, SLPs encountered dilemmas resulting from an expanded and expected scope of practice alongside issues related to that state rule.

Before working in a school setting, Ava treated patients with neurogenic disorders such as dysphagia (difficulty swallowing) and had worked with nurses and physical therapists in hospitals and nursing homes. Susie had worked at the elementary, middle, and high school levels and noted the differences in the skill sets required for each of these settings. Both women found that the required scope was a challenge for a couple of reasons. First, they were not necessarily trained in all aspects of the scope and second, they were not able to extend those services 
because of the nine-year rule which Ava believed to be unfair. Ava said it best: "I'm not sure ....can I practice or not??" and she added, "The lines are blurry... and you know kids could still use our help. Susie had another concern: "I'm not exactly trained in that, but they have us do it." Susie added, "You were expected to wear a lot of hats and do a lot of things that were maybe not necessarily within your actual job description." The connotation of "hats" was an easy way for Susie to express feeling overworked and confused in her role.

Ava, unlike many of the other SLPs in the group, maintained a private practice in which she treated patients in a medical setting. Hence, she knew more about working in the medical field, i.e. physical therapy, as well as how the staff responds to its demands. Based on that experience, Ava said, "Physical therapists specialize .... teachers have to specialize, right?" Trying to connect this point to her own situation as an SLP working in an educational setting, she referred to teacher certifications as a form of specialization — just as physical therapists have strong anatomical and medical backgrounds, middle school and high school teachers have concentrations in content areas like history and language. Ava seemed to be conveying this concept of specialization as something that might elevate her status in a school setting. Unfortunately, this idea of specialization has not transferred to the profession of speech and language therapists. Although universities are now offering course work in specialty areas, reading disorders and dysphagia, their licensure does not note this distinction. In RI, the education department is moving towards personal learning units (PLU's) for teacher 
accreditation and evaluation and are creating endorsement certification areas such as dyslexia. Perhaps, ASHA can be part of this direction as it would add value and significance to the SLP profession.

Patrice had worked in clinical and educational settings and had a son with autism; given these experiences, she had a more diverse background of knowledge compared to some of her other peers. With that background in mind, she half-joked, "We'll be delivering babies next!" Her comment reflected her view of the impossible range of tasks and responsibilities that SLPs are expected to undertake; by using hyperbole, she humorously and ironically defended her colleagues. According to Gee (2002), hyperbole is a device that is useful in conveying meaning, in coping with difficult situations, and in connecting with others. The use of irony is especially helpful as a defense mechanism, and as in this case, this exaggeration brings comic relief to a situation that is otherwise fraught with problems.

\section{ASHA Thrives, RISHA Exists}

Upon a state's request, ASHA's Board of Directors approves the implementation of a state-affiliated organization based on purposes that are consistent with ASHA's mission (ASHA, 2016). Approval for RISHA is at the discretion of ASHA's board. Therefore, RISHA would not exist without ASHA. Because it is the state arm of ASHA, RISHA adopts similar missions and codes of ethics. Unlike board members of ASHA's organization, however, RISHA's board members, other than the secretary position, are voluntary and unpaid. 
Consequently, efforts to recruit and maintain membership in RISHA is a challenge and for some SLPs, perhaps not a priority given their other work responsibilities. When RISHA's professional members and/or its board members become aware of a problem that Rhode Island SLPs are facing, RISHA often reaches out to ASHA for support since ASHA employs full-time lawyers who provide counsel to all of its state organizations.

While there may be some initial help available for RISHA issues, there is often a lack of continuing presence and support from ASHA. Case in point, RISHA was delinquent in distributing a survey in the spring of 2015 that was addressing a rule instituted in 2008. After RISHA contacted ASHA about a new state law that many SLPs objected to, called the nine-year rule, ASHA offered to disseminate a survey using its listserv. In the fall of 2015 , I became more involved with RISHA when I collaborated on a pilot study that involved analyzing survey responses of Rhode Island SLPs that included complaints about the nine-year rule. At the time of this writing in 2019, those survey results had not been shared with SLPs in the state. RISHA's reasons for its lack of responsiveness to publicize or share the results remain unknown, but I speculate that they are not sure how to proceed and/or not confident or motivated enough given the declining membership in the organization. When I inquired of the board as to why the results had not been shared, they responded that there was no longer a need since the rule had been implemented despite their opposition. Clearly, the state's 
legislative momentum on passing the rule was promulgated when ASHA and RISHA were slow to respond and/or organize against the rule.

One of those initial efforts instigated by ASHA was a recommendation that RISHA hire a lobbyist to promote the SLPs' opposition to this rule. ASHA also offered to pay any legal fees that arose from their opposition. Yet to my knowledge, RISHA has not hired a lobbyist. Again, I can only speculateperhaps, RISHA's board had come to accept the rule or maybe they do not have the time and/or the legal knowledge to proceed and organize such a task.

There was some evidence provided by one of the SLPs that may attest to RISHA's lack of involvement. It was clear that Eve, a RISHA member during some of this time period, had not been aware that Rhode Island was the only state with this rule in place when she asked the group, "What are the other states doing with this rule?" One would assume that an actively involved RISHA board would have produced a fully detailed report for its members of all the effects of the nineyear rule, including this very telling detail. It is possible that RISHA members' responses to the complications were sluggish because of certain variables, i.e. lack of knowledge on legal matters, being in touch with constituents, frequent changes in leadership; hence RISHA's reliance on ASHA for guidance as to what to provide its members. However, the results make it appear that RISHA is stagnant and idling.

Toniann presented another view of RISHA's history of activism. At the time of the interviews, Toniann had once been an active RISHA member, but 
not for a significant number of years. During her activist period, she had been part of a small and hardworking cohort that she felt had been effective in bringing about needed change. But she also highlighted just how much work it took to achieve their goals. She stated, "I wrote the licensing law for interpreters [sign-language]. We worked our butts off." She compared that group to the general membership of RISHA, saying, "You know, the passion is not there ...the let's-make-things-change isn't there and we'd rather ...too often sit back." She said:

Honestly, I don't think a lot of people know how the process works and how they can get involved or not. And it is time-consuming. I'm not saying it's not, because it's a huge time commitment. But do we know the process? Because even if you know the process, and we can get good, like, advocacy, you can send out-you can do email blasts - you can have an impact. And legislators in Rhode Island, because we are so small, really do listen to the constituents, you know?... I don't feel like we are proactive; I feel like we're more reactive. We wait until the regulations come up and then we say, "Oh gee, maybe that's not so good." In light of her experiences, Toniann offered commentary that was critical of RISHA and SLPs but also constructive regarding RISHA's future endeavors. In addition to the time demands, she knew the workings of policy and the course of action needed. Since the issues were particular to Rhode Island, she suggested that RISHA members should become more involved in legislative processes and learn 
the language and the process. Toniann does not blame anyone but offers an explanation. She speaks matter-of-factly because of her background knowledge of how both RISHA and ASHA operate in relationship to each other and their members, which in the case of ASHA, includes those of many other states as well as a larger scope of function. In reviewing Toniann's narrative about her work with her RISHA cohort, it would seem that for some time period, she felt that she and the other members had some amount of professional agency within the organization. She positioned herself as part of the RISHA group using the pronoun "we." However, this didn't appear to last, since by the time of the interviews, Toniann had dropped her membership in RISHA. Toniann conveyed her position switching out a pronoun with "you"; as such, she distinguished herself as someone experienced in politics. Without going into detail, she simply said, "RISHA didn't do what I wanted." Her matter-of-fact way of expressing her thoughts about RISHA may have had to do with her past experiences working in grassroots politics, which often requires this less reactive approach to outcomes. She did not personalize the aftermath of the rule by attributing it to something she did or did not do, i.e., attend hearings or write letters. She concluded with an "I" statement that signified RISHA was not effective and because of that she did not want to contribute. This appears to be trend as membership is declining. While she admitted lack of passion as an issue, she seemed to have solidified her decision to not enroll with the huge time commitment which is time-consuming. 
Overall, she was aware of the complexities behind the political scene; hence, the reason she was more accepting of where RISHA is right now.

Sandy's personal response was different. When responding to questions about RISHA’s support, Sandy stated, “RISHA—I don't need them at all; ASHA — it's kind of the same; I rarely use them except for resources." The tone of the words of her responses made it clear that Sandy, like Toniann, found the organization ineffective. In other words, a cycle exists in which SLPs are not contributing when an organization is operating ineffectively. Yet, in spite of her very apparent lack of enthusiasm about her memberships, she chose to remain as a member of both professional organizations.

Although differing in their personal ways of responding to the actions or lack of actions by RISHA and ASHA, Sandy and Toniann both agreed on two points: first, that their organizations acted inadequately when advocating for SLPs, and second, that they had to maintain their memberships in ASHA in spite of the cost and their complaints in order to be licensed to practice. In other words, for Sandy and Toniann, their relationship with ASHA was mostly an economical one that had to be maintained even though they both perceived ASHA as almost completely lacking in its role as a supporting agent of their profession. In terms of RISHA enrollment, Toniann could not be a member because she does not see them as useful and meaningful while Sandy enrolled because she likely connected and/or empathized with RISHA's struggles. 
If RISHA were able to step into the void created by this lack of professional attentiveness on the part of ASHA, its membership numbers might reflect that. But according to the interviewees, this was not the case. Rather than feeling as if they were professionally united through RISHA, most of the Rhode Island SLPs interviewed felt as if they were on their own to handle their problems. Unfortunately, inadequate responsiveness and a lack of support for policy changes did more to isolate SLPs than to unite them (Russell \& Kaderavek, 1993). Moreover, although the two organizations have similar mission statements, ASHA and RISHA do not maintain the same value in the eyes of the SLPs - they clearly expressed their need to maintain ASHA membership but not the same need to maintain membership in RISHA. Thus RISHA's partnership role with SLPs is fading, and in a kind of catch-22 scenario, its membership loss has prevented RISHA from stepping into the void that ASHA's mixed record of supporting its members has created. As such, SLPs are facing difficult challenges alone without the needed support of their organizations.

\section{Professional System Summary}

SLPs strive to meet their professional expectations, but this is ironically complicated by their professional associations. They are not comfortable in ASHA's multiple roles and binary function when it expects SLPs to be responsive, professionally and ethically, to a broad scope of practice with excellence and advocacy for their clients. SLPs were finding ways to contend with job expectations that surpassed their capabilities such as having a 
specialization area. ASHA has attempted to advocate for SLPs with creating a survey about the rule. However, SLPs feel unsupported and advocated for by their state organization. As a result, SLPs' relationships with RISHA are strained and, in some cases, may even be described as apathetic. On another note, some SLPs recognized and understood RISHA's limitations. In spite of having some empathy towards RISHA, SLPs are not vested in the organization and are less inclined to maintain membership than they are with ASHA. ASHA has the upper hand because of its primary function of credentialing, and so SLPs continue to subscribe to ASHA; due to this, it thrives.

In the following section, federal and local policies are described and analyzed in relation to the work of SLPs. In chapter 5, agency and identity are explained through positionality and socialization; both of which help to explain SLPs' navigation of dilemmas resulting from how these systems are entangled.

\section{System 2: Education Policies}

In this section, I describe and analyze complications related to policy, which according to critical theory, first requires policies be revisited. Next, I outline the complications that SLPs encountered: overlapping policies, driving forces in policy, i.e. numbers and cost; and lastly, policies' inadequacies in monitoring. Within those complications, SLPs experience frustration, alienation, and confusion. 
In order to better understand the systems-related challenges the speechlanguage pathologist participants in this study faced due to special education models such as the Response to Intervention (RTI), it is important to trace the promulgation of the federal acts that endorsed their use. The first federal act created to specifically address inequality of education for students with disabilities was The Education for All Handicapped Children Act of 1975 (Wright, 2010). However, this act included mandates from an earlier act of Congress, The Rehabilitation Act of 1973, which in Section 504 introduced FAPE, "free appropriate public education," as the standard necessary to ensure that disabled students received an equal education (U.S. Department of Education, 2010). The act also set out least restrictive environment (LRE) requirements that if followed, would ensure federal funding for schools. Over the next three decades, this act was amended and renamed several times in order to further elucidate the requirements that schools had to meet concerning FAPE and LRE. The first use of the title Individuals with Disabilities Education Act (IDEA) came in 1997 with an amended version appearing in 2004, commonly referred to as IDEA 2004.

In 2006, the federal government promulgated an additional series of "final regulations governing the Assistance to States for Education of Children with Disabilities Program and Preschool Grants for Children with Disabilities Program" (Federal Register, 2006, 46540). These final regulations of IDEA 2004 are referred to as Part B. They were meant to formally address areas covered by 
the earliest and subsequent acts that were raising questions and concerns by onsite professionals, including SLPs (American Speech-Language Hearing Association, n.d.). Although Response to Intervention (RTI) was not included by name in any of the legal mandates covered by any of these acts or subsequent regulations, it was a protocol already in use that fit the requirements of IDEA 2004 for determining a student's specific learning disability (RTI Action Network, n.d.). While efforts such as the adoption of RTI by many education systems to better comply with the education reforms laid out by IDEA 2004 have met with some success, they have also resulted in systemic inequities (Rinaldi \& Higgins-Averill, Stuart, 2011). For example, the outcome following the first appearance of FAPE regulations in 1973 for students with disabilities led to a major increase in the number of identified special education students and, therefore, higher student enrollments in special needs programs (IDEA, 1974, 2004[RP2]). The further specifications of the more recent IDEA 2004 and Part B created additional changes in key areas that have affected SLPs. Key areas of concern include personnel qualifications, evaluation procedures, IEPs, and early intervention services (American Speech, n.d.).

These changes have had positive results within certain statistical parameters, such as addressing the misrepresentation of students in special education, but they have also led to many problems outside of those parameters. With the advent of the new mandates, general and special educators began to coteach students in general education classes. This has worked well for some 
students but not for all, as according to one study, the best outcomes were achieved in models in which special education students had $50 \%$ or more inclusion in general education classes in their school day (Pearson, CavenaDeane, \& Supon-Carter, 2015), which is not the case in all schools. With time distributed between inclusion and intensive resource classes, special educators and SLPs had difficulty managing specific and unique needs of their students. Furthermore, with the onset of IDEA (2004) regulations, special educators, who were once recognized as the gatekeepers to special education, had been replaced by general educators through the inclusion of policies such as the general education initiative RTI; under RTI, general educators monitor students' progress and intervene as needed through the results of evidence-based practices (Fuchs et. al., 2010).

\section{Overlapping Policies}

Evidence from this study found SLPs encountered complications working within the RTI framework. They were unclear and confused on how to serve students challenged in their speaking and listening abilities. Some of the students had been discharged at the age of nine who had qualified under the discrepancy model. The manner by which these students became entitled to services changed with the RTI model. To complicate matters, the models of interventions differed within and across school districts. In the past a student eligible under the discrepancy model had an individualized education plan, now struggling students would fall under a recursive plan that consisted of three-tiered interventions. 
Under the discrepancy model, SLPs were case managers when the primary and singular disability was a speech sound disorder. With the nine-year rule, that was no longer possible for a student beyond the age of nine. As a result, those most familiar with the student and their type of disorder are not directly involved and the interventions likely delivered are not individualized nor aligned with their primary disorders.

Since SLPs are no longer case managers, they renamed caseloads as workloads which eventually became the accepted name. In fact, SLPs relied on ASHA's position papers which previously supported caseload caps and now have created workload formulas. This formula included direct and indirect ratios which helped SLPs manage their schedules. Their workload often included attending meetings for 504 students, another system which has also been impacted by the RTI movement. In fact, the American Disability Act had clarified and expanded the definition of 504 qualifications as a result of manifestation hearings in which parents wanted special education services. In one case a parent and lawyer claimed that without RTI, the student would be limited in his abilities and be impacted academically (ADA, 2011). In other words, RTI mitigated the effects of the disability. This speaks to the debate where parents find RTI to be ineffective and unfair when their children vacillate between good and bad grades because of how the recursive process works (Zirkel, 2011).

Susie noted her confusion about her job responsibilities. "I read up on IDEA, RTI, and ADA [American Disability Act] stuff ... and I'm not sure what I 
am supposed to be doing with kids. Can I screen? Can I evaluate? Can I discharge? Can they [students] have an IEP and a 504 and an intervention?" Despite Susie's review of policy, she was unsure of which role she should assume. Once IDEA 2004's language clearly opened the door that allowed states to select their own models for determining which students qualified for special education services (i.e., which students had learning disabilities), it was clear that there would be variable models of identification method. The unintended complications included manifestation hearings such as the case for one parent who argued for special education services when her child failed under the RTI process (Fairfield v Fairfax County School District, 2008).

Patrice, a veteran SLP, described her uncertainty that this variability produced. "With the discrepancy model, we had the wait-to-fail model — it is still a wait model except more waiting and watching and maybe more failing." Once again, loose policymaking and a subsequent lack of monitoring had not solved the issue of failing students. Susie noted her confusion about her job responsibilities. While Susie was uncertain about responsibilities, Patrice believed that students are still failing, and she is frustrated. It would seem that they are trapped in systems that were intended to help, but by design, have turned out to be restrictive in ways that prevent SLPs from fully carrying out their work.

The lines are hazy whereby SLPs do know the kinds of services that can be offered to students under different policy systems and, for a veteran SLP, she sees the flaws at work again. Based on the comments, whether there are one or 
two systems at work, the lines would be less hazy if policy makers respected the following considerations. First, policy needs to be aligned to context and with purpose; policies, old and new, need to be evaluated, debated and communicated, and finally, for successful implementation, there needs to be consensus and training for all stakeholders. SLPs are contending with legal, ethical and professional implications in their practice with policies framed by neoliberal policy makers who, unlike SLPs, do not have the knowledge and histories for making these changes (Lipman, 2005).

Clearly, there were variations due to conflicting and overlapping policies that presented complications and dilemmas for SLPs when they had to find ways to either qualify students for services or get them needed interventions. This situation was often equated with RTI's emphasis on data-driven results and tiered instruction. Ava, a veteran SLP teacher, complained that "things were very different then [before IDEA 2004] as far as the caseloads and what they looked like." She added, "When you made a decision, it was more based on the way we [had learned] in grad school." At the time of the interviews, Ava was working under the auspices of IEP-driven protocols alongside a RTI model and the nineyear rule, and it appeared that the new rules that governed her casework were greatly changing what she needed to do in order to serve her students in the ways that she had been taught in grad school. For example, Ava said, "You know before.... even with high caseloads there was staff, and back then we had team meetings [multi-disciplinary teams (MDT)] where we discussed whether we took 
students on." From Ava's commentary, it is clear that in the past, while she still had large caseloads, the operating policies were more collaborative, more streamlined with what she had learned in college.

This shift from collaboration to a separation into a kind of autonomy among special education professionals has accelerated because of yet another effort by the federal government to fine-tune policy in order to address ballooning numbers of students. In 2001, the federal government enacted the No Child Left Behind Act (NCLB, 2001). This act and IDEA 2004 conceptualized interventions differently and subsequently changed budgetary allocations (Fuchs et al., 2010). Although centralized and decentralized reform measures, as mandated by NCLB and IDEA 2004, were created to complement each other and further access to special education, their unification has instead created a have and have not situation whereby some students are privileged to receive special education and others continue to fail and struggle (Fuchs et a., 2010, p.304).

Because of this meshing of federal guidelines, states have come to interpret rules differently, resulting in more ambiguities and subjectivities that have changed SLPs work in terms of interactions with their students and with their colleagues. For example, Susie's complained, "How do I say no to this kid and not that kid ?"; while Toniann, a veteran teacher reported, "I'm trying to think of the number of referrals I've had from teachers this year. Maybe one...it's pathetic ... and getting worse... I find the students, they don't find me.” Hence, SLPs feel badly for their students that are not getting services and for Toniann, 
makes her question teachers' practices regarding the type of referrals she is getting. Toniann is not confident about the outcomes of having general education teachers assume the gatekeeping role in determining which students get services; hence, she no longer can rely on teachers for referrals. With the onset of IDEA 2004 and resulting programs like RTI, the special educators' role as decision makers for services, a role that their education provided them with, is being replaced by those who do not have that training (Fuchs et. al., 2010).

The problem with having general educators monitor students' progress and intervene as needed with evidence-based practices is that unfortunately, these systems do not monitor language and articulation growth. This matter became more complicated with Rhode Island's 2008 implementation of the nine-year rule. After the onset of this rule, students who were nine years of age or older and did not have a concomitant disorder could no longer qualify for an IEP. This was something Tim inquired about. 'I'm not sure how SLPs qualify students for RTI. I liked the discrepancy model; it was something concrete ... How do you qualify for RTI?" It appears that some SLPs are clearly still more in favor of previous policy as they are unsure of their role in the RTI approach.

To complicate matters further, at the secondary level, finding ways to deliver services was often a struggle for these SLPs. For instance, Ava explained, "Now when I evaluate, say, a 13-year-old that doesn't have an IEP, I make a clinical decision first, and then I say, 'Mmmm. How do I get him services?' I mean I make my own clinical decisions first, .... always.” Ava had to reconcile 
this procedurally created problem of being a clinician first, which she appeared to have done, but then she had to admit to the barriers in getting the services, and lastly, she felt obligated to question whether what happens next would be right for that student, adding, “...whether the outcome is right or wrong, I don't know." Ava was clearly uncomfortable. I compare Ava's dilemma to a doctorpatient scenario. When a doctor diagnoses a patient, he/she generally prescribes and delivers the plan of care. In contrast, Ava, who evaluated the student, does not get to deliver that plan.

Similarly, Kate added her ongoing hesitations about the best decisions for her students. "At the end of the day, my decision-making ... is it right or wrong? ..." then after a pause, she, like Ava, indicated that she still relied on past practices - "I am more confident with how we used to refer students because that is what I know. I treat RTI kids like IEP kids." The SLPs seemed to prefer one service model (IEP) over the other (RTI); perhaps, the policy was easier to follow and the other felt uncertain. In any event, this expectation of following RTI constrained the SLPs' agency. For instance, their decisions were based on policy of which they had no control and little information about, i.e. what kind of services can be offered and who will be delivering and monitoring it. Ava and other veteran SLPs had been trained to center treatment around their students' individual needs using data systems that measured speech and language.

This issue is not one that SLPs have fabricated. An examination of two of the regulatory acts that SLPs must comply with, IDEA 2004 and NCLB, reveals a 
historical timeline that shows that a plan was never developed to adequately address and monitor the needs of the most challenged learners (Fuchs et. al., 2010). When comparing the past with the present, SLPs' comments about their work under these policy changes revealed the transitions and implementations to have been ineffective because of inconsistencies in how staff was informed and prepared, in great part due to the fact that schools districts did not have the necessary staff and supports to provide that for them.

Many of the policy-related complications correspond with responses of the 340 SLPs surveyed about the nine-year rule (ASHA, 2015). For instance, $80 \%$ of SLPs had reported they were forced to discharged students with $36 \%$ of SLPs reporting that while some students were place in RTI, 59\% reported no services were provided and a small percentage $(5 \%)$ managed to continue IEP services. Also, when asked if the schools provided support after termination, $40 \%$ of the SLPs reported schools offered no supports, $20 \%$ were unsure, and the remaining $40 \%$ of SLPs offered resources and referrals to outside agencies. The SLPs' written comments were largely negative describing the rule as arbitrary and discriminatory with no evidence supporting rationale for the rule. Also, they complained that the rule contradicts Common Core Standards and that there was no monitoring system in place to monitor discharged students' outcomes. The intent of these policies might have been rooted in democratic values; however, poorly created policymaking that was not well thought-out and/or, according to Allington (2002), not monitored and/or aligned, undermined those values. 


\section{Numbers: A Driving Force in Policy}

SLPs are aware of the disparity for these students and SLPs like Tim had to find other ways to get appropriate services to students. Instead, Tim focused on balancing and managing his student numbers, i.e. RTI and IEP students. "There were caps with IEPs. Now there are workload formulas that cap off service for IEP and RTI students. The workload ...in a way, our work.... drives those numbers." Tim has struggled with managing numbers mostly because he is at multiple sites and in an urban district. He added, "RTI has lots of paperwork but IEP legalities take priority." He hinted that the IEP group of students was important; and, although the other group may be easier to work with, there was an abundance of paperwork. As such, Tim struggled with managing these two different types of students in which he seemed to prefer one over the other. Tim suggested his work drove numbers.

On another note, Patrice asked, "How do you generate your numbers?"

She knew I had fewer students at the secondary level and assumed that made my job less secure. Her tone suggested I had control over that and that I should think about generating referrals. Patrice's comment suggested she had control managing IEP numbers. Perhaps having control factored into her preferences which was what Tim alluded to when he said, "in a way our work drives numbers." In other words, their preferences for IEP students impacted how they worked. I got the sense that preferences for certain work happened because SLPs needed security and control. 
Patrice added, "You know, numbers and caseload types certainly drive policy, ... [but] .. .that's not all; they also drive the materials and resources, like the budget we used to have."

Patrice recalled the days when her department had a budget for buying therapy materials. According to Kaloi (2011), the Director for the National Center of Learning Disabilities (NCLD), 15\% of Title I monies had been reallocated in support of the general education initiative, RTI. Again, Patrice connected the dots between policies to economics when she noticed a drop in the special education budget after the general education initiative was implemented.

RTI was designed as a general education initiative; it became a special education problem. Tim's workload in an urban secondary school setting had been a challenge prior to the onset of RTI; since then, it had become even more difficult. He worked at the middle and high school and his number of IEPs and RTI students were high compared to the other SLPs in this study (See Appendix D). The RTI systems varied between the school sites and Tim had to adapt to those systems for those students all within a certain number of clinical hours. Previously, the state initiative placed caps on caseloads; now, that initiative applied caps to clinical contact hours, not to numbers of students. Based at an urban secondary level with so many struggling learners, Tim had to service more students with fewer budgeted contact hours, leading to shorter sessions. This is a service arrangement that is contrary to the profession's recommended protocol of high dose/low frequency treatments (Justice, Logan, Schmitt, \& 
Jiuang, 2016). Tim said, "I had to take more paperwork home and I am already behind." At the secondary level, students are on rotating schedules and they are not allowed to be pulled from certain classes, i.e. content subjects, gym and health which made this especially difficult to manage. As a result, Tim said, "I had to travel between schools.... and on some days, I had to return to a school I had already been at to see one more student." This district policy made it difficult for Tim to deliver the same services that might be possible under the differing policies of another district.

Clearly there are demonstrated links among numbers, policy, and resources. For one, policymakers made changes that supported cost reduction with replacing IEPs with RTI and second, that initiative decreased special education numbers. One of the SLPs assumed that belief when she connected speech referrals to productivity as a means to secure employment. Policies rooted in neoliberal principles rely on accountability, productivity, and cost reduction methods, and for SLPs this focus on productivity created complications in that they feel constrained in and/ or compelled to change their practices. Some SLPs offered their views on what they assumed were the causal triggers for their problems, for instance policy or workload numbers, and they differed in their understanding and consciousness of those triggers. Ava conceived of policy and workload using a metaphor. "Is it the chicken or egg thing?" In other words, Ava saw this dilemma as interconnected and never ending, maybe unresolvable. On the other hand, Patrice was compelled to generate work numbers in response to 
policy. In either case, neoliberal influences were not exactly redressing inequalities.

\section{Policy Fails in Ensuring Success in RI}

In order to establish the success of any educational policy, policymakers seek "evidence" to determine whether policy is succeeding or whether further change is needed. For example, while national testing of pupils is a form of evidence that seeks to assess and ensure student learning (Bodman \& Taylor, 2012), research on RTI confirm universal data processes conducted with fidelity and consistency ensures student responsiveness (Fuchs et al., 2010). However, Rhode Island policymakers have not sought evidence against a rule nor devised a data system that would monitor oral language for students with speech needs. Yet, the SLPs in this study raised the fact that there are great inadequacies in the way that the RTI model has so far ignored measuring speech-language capabilities of these students. Some SLPs also felt that tracking data on students impacted by the nine-year rule should be implemented since they now fall under the RTI monitoring system. SLPs voiced their concerns that these students could be experiencing academic issues related to the rule. Kate said, "We [SLPs in her district] started a tracking system to check on these students but we stopped." Tim's comment was more positive. "We have been tracking and we see correlations between referrals and declining grades." Patrice was intrigued by this and added, "You know that's a good idea. We should do something like that at the state level." Since there has been no state action, specific districts have taken the 
initiative to monitor the academic progress of those students discharged from special education services at the age of nine in the hopes that their statistics will provide the state with the evidence it needs to address any problems that have arisen as a result of that rule.

Research states compliance with policies should include monitoring systems but in RI that is not the case. McGuinn (2010), a policy professor specializing in school reform, stated that policies once approved should have a process in place that monitors effectiveness. Some of the Rhode Island SLPs are already aware of the negative effects of RTI and the nine-year rule on their students and have attempted to collect data that could prove that the rule is unfair and discriminatory. Tim described efforts within his district, "Our department has been tracking the students who were discharged at the age on nine and with all this data, we should be able to do something with it." Upon further questioning, he said, "We really haven’t used the data for anything." Patrice added, "That evidence would be instrumental if we could get it across the state." Kate was somewhat rhetorical, "Well the state made the rule so ... shouldn't it be the state be managing that?" In line with that lapse, Eddie suggested, "Unless we are talking to administration...or ..." Eddie was suggesting SLPs do something beyond talking to their district administrators. From the data analysis of this study, it is clear that while SLPs are trying to find ways to address policy problems on a local basis, there is less evidence that they are being supported by their districts, and last but not least, the state. Not knowing if what one is doing 
matters, it is easier to question one's efforts in trying to change policy. According to Eddie, change is possible and would require that SLPs communicate to a higher authority.

SLPs were addressing the problems related to policy using whatever means within their power. Kate addressed the early dismissal of students from special services under the auspices of the nine-year rule greatly differed from some of the other SLPs and may have had unintentional results. "I just pick kids up earlier, under the table....you know, because I know they are going to get discharged." Although she carried out this practice with the best intentions, the outcomes for this solution to her problems could have less desirous results. For one, not all students respond to early interventions since developmentally, they may not be ready. For another, because this is a kind of "off-the-record" speech therapy, the records may end up crediting a different intervention that the student received as the basis for his or her progress. Kate's words, under the table, seemed evasive, as if she was stealing, and her tone conveyed guilt. In any event, data monitoring patterns for that student would be skewed and invalid and that outcome is disconcerting.

Patrice took another precaution which for some could be perceived as risky, she said, "I make students eligible under another category...I basically collaborate with a teacher and build a case making a student eligible under a written disability." Before the nine-year-rule, Patrice said, "I didn't have to do this, the student could qualify under oral language." Patrice's actions could be 
perceived as illegal, perhaps unethical. However, she explained, "You know that kid is eventually going to have written issues if he doesn't get help now." Later, she seemed compelled to defend herself, saying, "I have been forced to do these things." Ironically, this act violates the empirical-based model on which medical practices and RTI are premised. Patrice is a trusted SLP and has been working in this school for 15 years; as such she was able to accomplish this maneuver with a collaborator, the elementary teacher.

Like Kate, Patrice circumvented a system to help a child. While they subvert a system that is harmful or neglectful to their students with speech needs, their actions have potential consequences. They are in the position of deciding what is right for a student versus what makes data accurate and valid. Unfortunately, this is a situation one would not want to be in for two reasons: 1) SLPs want to follow protocol in spite of the systems that go against their mission and 2) the consequences of their actions have negative outcomes. These unintentional results have been forced upon them while they attempt to help children.

\section{Policy System Summary}

The complications associated with multiple overriding policies create dilemmas in which the SLPs felt confused, frustrated, and unprepared. For instance, the political and cultural shifts to which policymakers respond resulted in changes that do not align with SLPs' training, work experiences, and expectations in various settings. While the intentions of policymakers may have 
been to address political and societal issues, they do not adequately adjust for and acknowledge state and institutional differences. As a result, federal and state policies made SLPs' work more difficult and constrained their practices. In the state of Rhode Island, SLPs are doing more to track the effects of the nine-year rule. SLPs are attempting to circumvent issues related to bad policy like making a case for another disability or making a student eligible for therapy before they are developmentally ready. The complications related to generating and managing workloads created struggles which impacted their relationships and perhaps promulgated other problems such as skewing data.

The first complication found SLPs confused as a result of overlapping policies. The differences between policies mattered in terms of who received speech services and how often students would get those services. They believed the policy changes influenced the number and type of student referrals which also impacted their relationships with general education teachers and parents.

A second complication related to policies is the creation of a have and have not scenario in which SLPs struggled with which students receive services. A review of policy revealed correlations between numbers and special education enrollment. On one hand, policy was a corrective measure for reducing costs related to high special education enrollment; on the other hand, it was a welcome relief for SLPs who had the burden of large caseloads from previous policies, i.e. FAPE and IDEA. 
A third complication stems from policymaking which fails to adequately monitor and ensure policy is working. In the state of Rhode Island, SLPs are doing more to track the effects of the nine-year rule. SLPs are attempting to circumvent issues related to bad policy like making a case for another disability or making a student eligible for therapy before they are developmentally ready.

These complications are the culmination of what happens when policy intersects with societal and cultural conditions. Certain events and conditions legitimized policy that varied state to state and district to district. Unfortunately, the participants, SLPs, teachers, and students, are not necessarily aware of the reasons behind policy and, to some extent, their responses and frustration do more to entrench complications making them less recognizable.

\section{Chapter Conclusion}

The first system, consisting of professional organizations ASHA and RISHA, are repositories of shared values, mission, advocacy, and excellence in practice. However, their memberships are declining and SLPs feel they are not supportive. The second system, education policy, has shifted to a neoliberal focus that is concerned with cost, productivity, and accountability (Lipman, 2005) over student well-being. In this political scenario, SLPs feel they and their students are marginalized and less valued; yet they respond with less-preferred interventions, i.e. picking up students earlier. These systems do not happen alone but are bound to context. 
The following chapter has two sections: Context of the Education Institution and SLPS'Navigation of Dilemmas in the Entangled Systems. The context and SLPs' relationships are discussed in terms of complications with an emphasis on navigating the dilemmas that constrain SLPs' agency. The navigational processes are explained through SLPs' discursive practices in which they locate themselves within a multi-directional compass mostly guided by emotion. 


\section{CHAPTER 5: ANALYSIS}

Chapter 4 detailed the complications that arose for a sampling of Rhode Island's speech language pathologists (SLPs) when following the prescribed policies dictated by the directives of the state's nine-year rule concomitantly with the regulations of federal policies, i.e., the Response to Intervention (RTI) and the suggested policies of their professional membership organizations. An analysis of the interview data demonstrated that these complications stemmed from the entanglement of the agency systems involved, not simply from the individual actions of these agencies. However, it was also apparent from the interviews and group discussions that, in many if not most situations, the participating SLPs were unable to properly identify the source of their problems. They attributed the source of their dilemmas on one or another individual agent, instead of seeing that the problems stemmed from a complicated chain of actions involving at least four "systems": SLP professional organizations, state and federal policies, the education institution, and the parent body. All these systems acted independently on behalf of the welfare of the students. Yet the outcome of many of the systems' seemingly benevolent actions caused problems for these SLPs that prevented them from performing their jobs in a manner that complied with their professional expectations. I refer to this situation as system entanglements.

\section{System Entanglements' Complications and Dilemmas}

This chapter describes the mechanism of system entanglements within the context of educational institutions in Rhode Island and how these entanglements 
affected the working conditions and personal responses of the study's participants. Guided by the theoretical frameworks outlined in chapter 2 and an application of methodologies discussed in chapter 3, I examined the complicating factors that led to the complex dilemmas that SLPs had to navigate. These entanglements were rooted in the areas of politics, economics, and culture. According to critical theorists, it is the understanding of the roots of any issue that contributes to a resistance or endorsement of certain acts at a specific time within a specific institution (Quinn, 2012). Gee (2011) adds that within those institutions, participants' discursive practices were the medium that either constrained or enabled their agency.

In this study, the SLPs lacked a true understanding of the roots of their dilemmas. Although every problematic situation they discussed was complicated by multiple systems entanglements, many of the SLPs failed to see that. Instead, they experienced each as having occurred as a separate action that was independently causing complications for them. As the unacknowledged entanglements grew, the dilemmas broadened, increasing the SLPs' frustration at being unable to solve the problems in ways that they were familiar with. SLPs' navigation of these dilemmas can be explained in part by Gee's (2011) building tasks and social recognition (2001), Bell's views on oppression (2007); Holland et.al's (1998) conception of agency, and Boler's (1999) Pedagogy of Discomfort, which are all described later in this chapter. 


\section{An Example of System Entanglement}

The following is an overview of one issue that involved all four of the systems represented in this chapter. The SLPs were concerned that:

1. As a result of new federal and state regulations, SLPs were required to observe new rules when serving students;

2. In assessing these new rules, the American Speech-Language-Hearing Association (ASHA) had advised its members of the efficacy of broadening SLPs' scope of practice even though this directive extended the duties of SLPs far beyond the time available during the school day and/or beyond the SLPs' training and experience levels;

3. School administrations, following the new regulatory mandates (and with no rejection of these policies by ASHA and RISHA) began to rewrite work expectations for SLPs that greatly complicated their ability to properly serve their students; and

4. Based on these new practices, parent expectations on behalf of their children began to include outcomes that were virtually impossible to attain because of the student eligibility policies set in place by both federal and state regulations.

Expressed in a flow of cause and effect, it is clear that there was actually no way to separate out one ripple from another in terms of the end result for SLPs. However, in their discussions about these issues, the SLPs blamed the increasing number and complexity of their cases, the lack of resources, and the added responsibilities on one or another of the four systems. Depending on who was 
speaking, he or she blamed one of the individual systems but never the interactions of the systems.

Adding to this confusion was the fact that since each of these systems purported to enact policy or ask for services that supported students, SLPs often felt professionally and personally obligated to tolerate the complications, leaving them with very few options but to try and work harder to achieve what they knew to be impossible. Unfortunately, as the entanglements continued, the complications and dilemmas accumulated-like a stone thrown in water, these merging systems triggered a ripple effect whereby the ripples widened, making what lay at the bottom less recognizable. Because of this, SLPs in the study did not necessarily notice or understand the roots of the problem even though the first system discussed is obvious, the very institution that they worked in.

\section{Educational Institution: People, Places, Procedures, and Protocols (4 P's)}

Archer (2000) states that whether one chooses to endorse or resist certain acts depends on when and where participants are situated in the process. In this study, the SLPs' practices and interactions with students and parents were constrained and that constraint varied in regard to the school they worked in. SLPs were expected to observe procedural changes stemming from regulations that varied by setting and the particular administration's interpretation of these regulations (Allington, 2002). Through the nature of their work, SLPs engaged with multiple stakeholders, colleagues, and administrators who were all accountable to academic standards on behalf of students with special needs and parents of those students. Because SLPs valued the perceptions of parents, 
colleagues, and administrators as it related to their work, they were challenged when their preferred practices conflicted with those of the stakeholders. Much of this shift in the SLPs' positioning resulted from the university or college programs that did not necessarily prepare them for the situations they experienced with their eventual jobs. As a result of this interface of factors within the education setting, major complications emerged when the four P's-People, Places, Procedures, and Protocols - had to be taken into account as both an individual and entangled system. When SLPs carried career-related issues forward into their work setting, they encountered situations that either suited their perceptions or constrained their agency. For example, they made students eligible under another disability, serviced them informally, or referred them to outside agencies.

\section{Constraints on SLP Agency}

The following section contains an overview of system entanglements stemming from mandated policy that triggered a chain reaction and accumulating complications that constrained SLPs' agentive capabilities. The complications created ethical and professional paradoxes because the participating institutions established their principles of operation on different sanctions: legal, economic, and political (Bell, 2012). Lipman (2005) claims that education research on accountability, productivity, and cost reduction exacerbates education inequality and that these policies contribute to broader social and economic inequalities. A policy's underlying and competing principles of productivity and accountability have created paradoxical scenarios (Clarke, 2005) and have or have not scenarios (Tilly, 2003). Nygreen (2013) adds that although policy reform intended to make 
education more accessible, the push for accountability has instead constrained teachers' abilities to differentiate instruction. Due to this, some students do not have full access to services.

Any attempts by this study's SLPs to address the most basic expectation that all students must have access to the available services were thwarted by an entanglement. By examining the data describing their observations of one of the four systems' issues — in this case federal and state regulations and their subsequent revisions - it was easier to see the chain effect of system entanglements since the SLPs could not describe the ensuing dilemmas caused by that system without bringing into the discussion at least one of the other systems. For example, elementary school based SLPs require parental assistance in achieving student objectives. Based on this requirement, system entanglement \#4 -parent expectation- became part of the picture. Carrying out this particular analysis of the effects of regulations on the work of the SLPs indicated that an overview of the named systems resulted in accumulating complications. With each system, I identified two to three complications. However, the last system entanglement I found only one complication. I was hesitant in naming parents as part of a contributing system because they were subjected to restrictive policy. Regardless, the trajectory of reform also implicated parents which subsequently shaped the SLP's professional identity and agency.

While I applied theory to explain the SLPs' accounts of and responses to these complications, theory and method also illustrated the SLPs' navigation of the dilemmas (See Table 4). Those explanations included the following 
theoretical frameworks: identity and agency in cultural models, critical theory and adult transformative learning, and critical discourse analysis (CDA). By applying critical discourse analysis and rhetorical analysis, I conceived of how language functioned in navigating dilemmas for each identified system.

Table 7. System Entanglements

\begin{tabular}{|c|c|c|c|c|}
\hline $\begin{array}{l}\text { Trigger: the } \\
\text { rule }\end{array}$ & $\begin{array}{c}\text { Complication } \\
-1-\end{array}$ & $\begin{array}{c}\text { Complication } \\
-2-\end{array}$ & $\begin{array}{c}\text { Complication } \\
-3-\end{array}$ & $\begin{array}{l}\text { In dilemmas: language } \\
\text { functions to relieve } \& \\
\text { recruit SLPS }\end{array}$ \\
\hline $\begin{array}{l}\text { Policy } \\
-1-\end{array}$ & $\begin{array}{l}\text { Ethical and } \\
\text { Professional } \\
\text { Paradox }\end{array}$ & $\begin{array}{l}\text { Sanctions, } \\
\text { legal and } \\
\text { economic, } \\
\text { threaten } \\
\text { standards }\end{array}$ & & $\begin{array}{l}\text { Being a } \\
\text { Professional is } \\
\text { rationalized } \\
\text { as following } \\
\text { the law } \\
\text { despite the } \\
\text { ethical } \\
\text { tension (Gee, } \\
\text { 2011) } \\
\text { Emotional } \\
\text { declarative } \\
\text { and active } \\
\text { voice, and } \\
\text { past tense } \\
\text { showing } \\
\text { agency, } \\
\text { important to } \\
\text { professional } \\
\text { integrity } \\
\text { (Boler, 1999; } \\
\text { Gee, 2011). } \\
\text { We/our and } \\
\text { you know to } \\
\text { create } \\
\text { solidarity and } \\
\text { acceptance } \\
\text { for what they } \\
\text { perceive is } \\
\text { their only } \\
\text { option (Gee, } \\
\text { 2011). }\end{array}$ \\
\hline
\end{tabular}




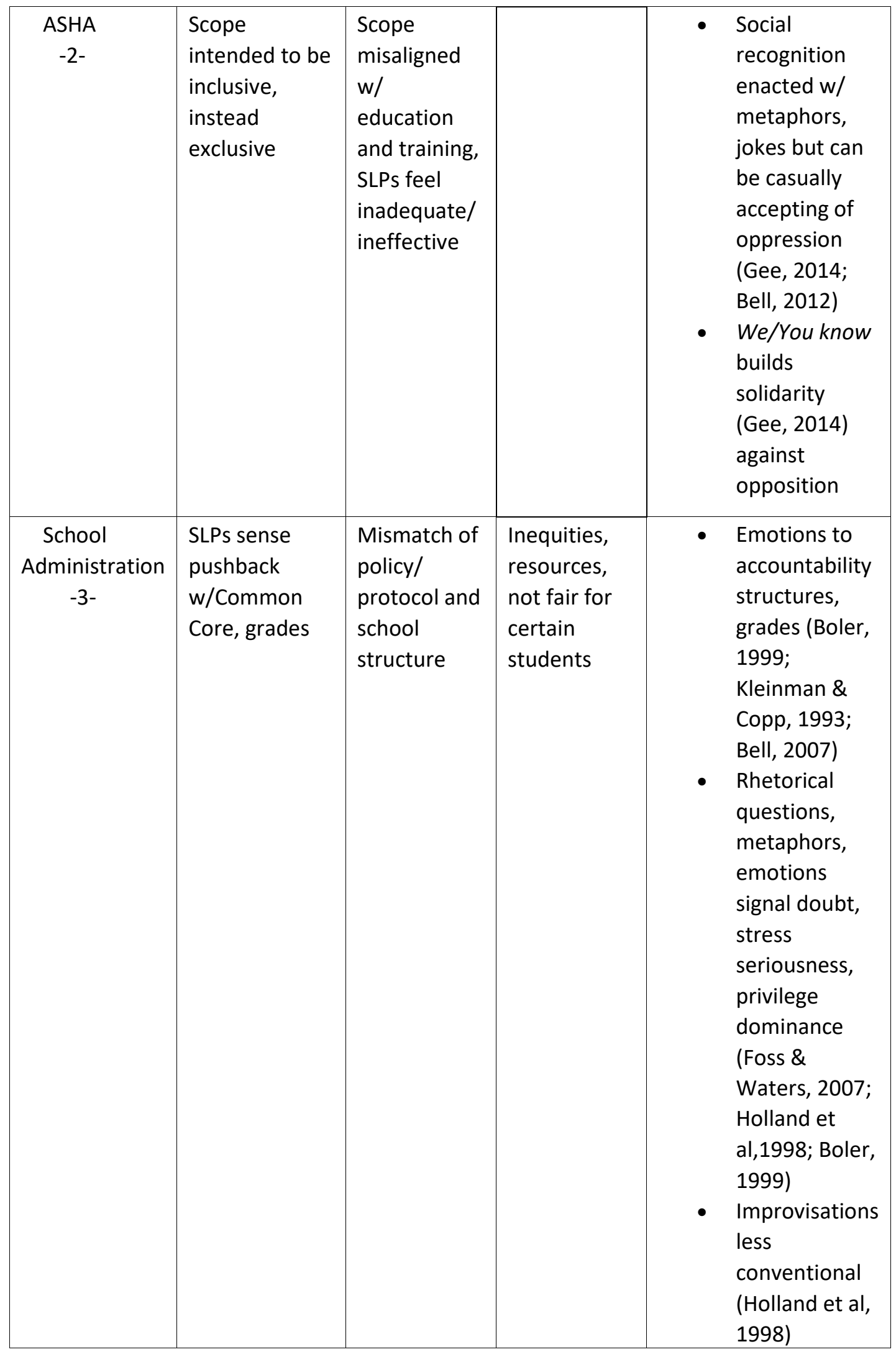




\begin{tabular}{|c|c|c|}
\hline & & $\begin{array}{l}\text { You know, } \\
\text { solidarity, in } \\
\text { welcoming a } \\
\text { rule (Gee, } \\
\text { 2011) that } \\
\text { overloaded } \\
\text { them }\end{array}$ \\
\hline $\begin{array}{c}\text { Parents } \\
-4-\end{array}$ & $\begin{array}{l}\text { Parents- } \\
\text { demanding, } \\
\text { uncooperative }\end{array}$ & $\begin{array}{l}\text { - Parents, } \\
\text { subjected to } \\
\text { rule, yet } \\
\text { implicated in } \\
\text { system } \\
\text { - Powers } \\
\text { remain hidden } \\
\text { - parents } \\
\text { blamed (Gee, } \\
\text { 2001) }\end{array}$ \\
\hline
\end{tabular}

\section{Entanglement 1: New Federal and State Regulations}

\section{An Ethical and Professional Paradox}

In the following scenario, the SLPs felt obliged to follow the law despite knowing that this obligation conflicted with their university training and the standards set forth by their professional organization, all of which they preferred to adhere to as professionals (Eraut, 2008; Branch, \& Frankel, 2016). The participants tried to find ways to serve students who were no longer eligible for speech under the auspices of a particular Rhode Island regulation (the nine-year rule). Because they also had to observe the restraints of the federally mandated revisions to IDEA 2004, which determined that students would be monitored under a universal monitoring system (RTI), having to follow the nine-year rule created even more complications than it would have without RTI in the picture. In 
the following statements made during this study's discussion sessions, various SLPs defended their actions claiming that lack of resources and professional duty guided their decisions, while also expressing their lack of ease with the differences they encountered between their required actions and their professional expectations. This dichotomy subsequently triggered certain responses.

Toniann found a way, albeit not the usual one, to serve her students during school hours to the level she felt they would benefit as well as the level of work that she expected of herself. When other SLPs were asked if they had resources in guiding or helping them manage their workloads, most felt that they had few choices in terms of options. As a result, they chose to follow the law rather than adhere to their deeper understanding of what was needed for a particular student. Ava said, “One voice doesn't matter...I can't beat the system. I have to follow the law." Toniann agreed, "Professionally I follow the law. ... Ethically, do I feel good about it? NO, not really." Her admission reflected the opposing inner voices that she had to contend with much of the time, something that many of the study's SLPs experienced. She defended her positionality even as she saw this new way of responding to her work as a step off the path of professionalism.

Ava and Toniann found themselves in a professional and ethical paradox because professionally they were not working to expected standards. Moreover, meeting those standards was problematic with restrictive policies and the lack of school supports and resources. While SLPs clearly saw the havoc the nine-year rule and RTI were causing — for their students and for them — they complied with 
the laws because in their opinion to do otherwise would have been unprofessional. Toniann used a rhetorical question to bring attention to a bad rule; yet, she legitimized it with a professional identity that sanctions SLPs adherence to the law. To normalize this version of professionalism has ethical implications in the SLP profession. While SLPs did what they were expected to do by the administration (and not what they had been taught to do), they were agentive in getting services in less orthodox ways, such as during lunch time or making students eligible under another disability.

\section{Sanctions, Both Legal and Economic, Threaten SLP's Standards of Practice}

The study's veteran SLPs, with their historical perspectives, often had more reasons to worry about the future of their work ethic. Toniann said, "Remember in the past, the state cut all related services, OT PT ST [occupational, physical, and speech therapy], in the behavior sector." The behavior sector included mental health sites that provided inpatient and outpatient services. Toniann explained further, “They did it to save money.” Looking at what was happening in the present, Toniann was worried about the implications of the nineyear rule having to function alongside existing Medicaid cuts. She was basing her predictions on her familiarity with past bureaucratic decisions that were driven by state budget changes. "Now the nine-year rule.... the Medicaid issue is a more contentious issue... money is cut, workloads are still high. But SLPs will be cut because our services are no longer Medicaid reimbursed." Toniann predicted changes. "With less staff and the same amount of kids... our standards will change." 
Toniann went on to describe how little the public's input actually mattered in bringing about change. "There is so much more work behind the scenes...rules are passed by the time the public has anything to say...hearings and forums are perfunctory. [T]hey waste your time." Another veteran SLP, Ava, was just as pessimistic. "In Rhode Island, our state deals with red tape constantly...very typical in this state. My voice does not make a difference."

Not having a voice was a major problem and SLPs believed these regulations could affect the integrity of their work. Maintaining a high degree of integrity is essential in an SLP's practice as they are responsible for ethical codes and a mission of advocacy. Toniann shared a past experience in which her personal notes were used against her work in a due process hearing. "I remember way back a past case when one of the agencies I was working with, we had all of our records subpoenaed and personal notes were used to support them not me. Now, I document minimally, differently." Toniann explained that she had felt personally attacked when the lawyer had used her notes to insinuate that the student had been making minimal progress as a result of inadequate and ineffective speech therapy. This experience led her to change her methods of documentation so that her notes could not be used to question her abilities. Although this action could be seen as agentive, she perceived insult and that experience compromised her self-worth. In fact, Toniann had some concerns about having had to take these steps, leaving her with a feeling that she had compromised her professional and personal integrity. This impacted her sense of self, her identity as an SLP, challenging her sense of value as seen through the 
eyes of the parents of her students, the eyes of the administration of the school, and, at least temporarily, through her own eyes.

Another SLP, Sandy, who also changed her documentation methods, shared that she had always felt confident about her work but after being questioned and challenged in a due process hearing where the parents felt that their son had not been getting enough services, her whole attitude about herself

and her work underwent a major transformation. "I am good at my job .... I used to care—now, it's hard to." Her reactions to legal sanctions were perceived as personal attacks. Now she vacillated between caring and not caring. SLPs' perceptions of their value yield emotional responses that can drive or inhibit results for their students.

\section{SLPs' Navigation and Agentive Processes}

According to Gee (2014), language functions to signify identity and power; in this case, the term professional conveyed SLPs' position in a hierarchical as well as a respectful manner. According to Boler (1999), a disclosure is a mechanism to excuse one from any liability. Likewise, SLPs claimed professional duty as a form of pardon or dismissal of liability in case something went wrong. The hierarchical and respectful use of professionalism positioned them normatively despite their feelings of being coerced. Boler (1999) states that feelings are catalytic and, in this case, SLPs voiced discomfort, which is a transformative step in navigating ethical dilemmas.

According to Fields (2012), agency is slowed down when educators are positioned as vulnerable and disempowered (as cited in Izadinia, 2014). Both 
Sandy and Toniann expressed having had this experience when they shared that the due process hearings, which were about frequency of services, had become personal and punitive attacks on them. Grammatically, Sandy spoke in the first person; hence she was declarative and took ownership, speaking with conviction. Her use of the past tense demonstrated her belief that her practices had gone from good to less favorable. The pause between her statements revealed her hesitance, for she knew what she was going to say would not necessarily be something perceived as favorable. Toniann and Sandy were agentive with changing the way they documented. Yet, they maintained a concern that their responses to this dilemma could potentially harm students because their notetaking was no longer authentic. Of greater concern is the self-protective stance that Sandy took when she excused herself for not going over and beyond as she had in her previous practices.

According to aspects of professional identity formation, experience, reflection, and reflexivity shape a professional's beliefs and actions (Johnson et al., 2013). Toniann's accounts of past experiences of regulatory cuts were instrumental to discussions on SLP standards. The veteran SLP warned focus group members that standards would continue to change with the nine-year rule. Toniann's grassroots work taught her that policy work was complex. This discussion led to the topic of the role that SLPs' professional organizations must begin to acknowledge and undertake because of the situations SLPs found themselves in. 


\section{Entanglement 2: ASHA's Response to Policy}

The SLPs in this study struggled to maintain standards of excellence in the face of legislative changes. Their identity kits, i.e., their college years and training that they relied on in previous experiences were being dismantled (Gee, 2014). Moreover, these experiences were especially disorienting since their professional organizations, which they felt should have been advocating and supporting SLPs, were partly contributing to the problem.

The American Speech-Language-Hearing Association (ASHA) expects SLPs to work with students who are diagnosed with a broad scope of disorders. Within the educational setting, ASHA recently expanded the SLP's role in the area of literacy when policy initiatives surrounding declining literacy scores were being promulgated (Nygreen, 2013; Lipman, 2005). As a result of this role expansion, SLPs have encountered complications that challenge their professional ethics. A serve or do not serve scenario has resulted in situations whereby certain students are not getting adequate services (Lipman, 2005).

\section{Scope Intended to be Inclusive, Not Exclusive}

Rather than helping the SLPs find ways out of their ethical dilemmas, ASHA supported further expansion of duties and responsibilities that engendered the possibility of more ethical dilemmas. When employer expectations conflict with personal work standards, jobholders often turn to their unions, human resource supports, and professional organizations for guidance (Bolman \& Deal, 2013). Usually, the organization stands behind its members in upholding the 
tenets of the profession, often by lobbying for policy that better supports its members' mission. According to the discussions of the SLP focus group, ASHA had not undertaken this role on a national level and RISHA, the state's ASHA affiliate, similarly did not offer support on the state level. In some cases, ASHA's statements of intent for its members seemed to be a major cause of the increase in employer expectations and a source of members' problems. A case in point was that ASHA promoted an increased scope of practice for SLPs, which had created major conflicts for many of the study's SLPs due in part to their need to comply with Rhode Island's nine-year rule. RISHA, understaffed and underfunded as it was, did not attempt to engage its parent body ASHA on this point, thereby relinquishing its role as a support agency for its members. So, instead of having only one stakeholder agency with whom their views conflicted (the regulation system), this group of SLPs found themselves on the opposite side of the table from their own professional organization system.

The SLP discussion group participants who were members of RISHA shared that in the past they would have described their work ethic as good but eventually had discovered that they faced some challenges to that belief. Their discussions concerned a conflict between the scope of practice policy and their ability to work with a particular disability that was included in that scope. Some of the SLPs had had little experience working with students diagnosed with dysphagia, for example, yet as one of the SLPs reminded them, it was, in fact, within that prescribed scope. Eddie's comment on the subject was one of resigned agreement with the policy. He remarked to his fellow SLPs that "Dysphagia is 
part of our scope of practice. ... whether we feel comfortable [with that] or not." Sandy said, "My problem with dysphagia students is that we need nurses on site for the more complex cases and....my school does not have that support." Since neither ASHA nor RISHA had done any meaningful work on behalf of its members to change the policy that extended the scope of practice and therefore the already impossible number of cases its members had to cover, these SLPs had nowhere to turn to except to covert actions on the job in order to meet their professional standards of practice for their students. And this left many of them worried.

One SLP worried that some of the practices she felt she needed to follow were not even legal anymore. Kate said, "We can't even screen, I mean, you know ... at least legally." Eddie's response drew laughter from the group when he jokingly responded, "What are you? Worried? You'll be jailed. ... I'll read about you in The ASHA Leader under 'Ethics Review."

While Kate was attempting to assess the group's thoughts on the matter, it was clear by their laughter that they probably felt guilty by association to some degree. Perhaps they too had been forced to covertly perform services necessary for the welfare of their students and were concerned about being accused of ethical violations. In any event, their laughter and dark humor suggested that these SLPs were struggling with restrictive policies set in place by regulations (one system) and promoted by their professional organizations (another system) that did not align with their own professional mission and scope of practice. 


\section{ASHA's Scope Not Supported at Work nor Consistent with Training}

To practice as a speech-language pathologist, aspiring students must learn about communication science in accredited universities. If universities do not align knowledge and professional standards with current work needs, they open the door to potential losses for those receiving services as well as for those who are offering the services. According to Johnson, cultural mismatches such as institutional differences in mission statements and goals influence SLPs' agency when they inhabit a new setting (as cited in Ullman, 2012). These moments can be transformative when SLPs find solutions around those misalignments.

Although addressing institutional differences matter, Bell (2007) would also advise that participants examine the interpersonal, interrelation, and internal parts of the emancipatory process. By reflecting and sharing their experiences, beliefs, and feelings, SLPs learn about the relational and contextual influences and personal contributions that complicate their practice. In doing so, they can work towards change in themselves and unite against dominant forces.

After completing their university coursework, SLP students are placed at clinical sites by their colleges, much like education programs place pre-service teachers at school sites. These placements are an important part of an SLP's training for the development of his or her skills. Placements are important in shaping an SLP's perception of future opportunities. Therefore, they can either enable or constrain the SLP's career path. 
According to Susie, her clinical practicums were contrived and unrealistic. "At the University Clinic, you interacted one-on-one with students and conferenced with your supervisor regularly. We worked in a room with a oneway mirror [so that] I could be rescued at any moment." Susie was referring to the speech therapy room at the university clinic in which supervisors sat behind a two-way mirror and observed SLPs' sessions with their clients. After the session, the supervisor conducted a consultation with the SLP and provided him or her with a written evaluation. Susie believed that the required 375 clinical hours of training were helpful in obtaining specific experience with various populations and students, i.e., children with fluency issues. Susie felt that this experience was valuable in some ways but that it was too orchestrated and controlled to be realistic. Case studies that are hypothetical and predictable (and becoming more popular in telepractice sessions) are not preparing students for the realities of the job. Caroline recalled certain assignments that echoed this belief. "In college, we discussed case studies which were hypothetical therapistclient scenarios [that were] nothing like the work we do....here. [In the workplace], we have concurrent and complex diagnoses."

At Caroline's present job, her speech groups consisted of students with different needs and from various grades. She felt that her training had not prepared her for the reality of the public-school setting. She also felt that the client-SLP interactions were manufactured since they were just case studies and as such lacked authenticity. She had difficulty organizing groups and scheduling students by grade and needs. The newly graduated SLPs felt that would have 
benefitted from a more realistic setting than the lab environment or studying hypothetical scenarios.

Caroline had earned a master's degree in Communicative Disorders just one year prior to the interviews; she came into her position at her school with only her training to rely on. When she needed detailed information and perhaps even the options that were open to her to help her understand her duties and her role as an SLP in the school, she reported that she was sometimes given only the most minimal pieces of information. She explained, "I was taught about the nine-year rule ... and that eligibility under a speech-language impairment was no longer possible on a child's ninth birthday. So speech services end. That's all I know.... [W]e learned mostly about common core standards and federal legislation.” Caroline did not question the regulation; she just assumed that it was what it was and that she would have to find a way to accept the fact that the students' improved work in the common core subjects overrode the need for therapeutic services, even during her assigned time with them. She said, "In every single session I have a lesson planned. But if a kid walks in and their goal is vocabulary and they have an English class next period, my lesson is out the window. Like my lesson doesn't matter." She encountered the problems of policy when they overlapped with the expectations of teachers, i.e., standards, common core, grades.

SLPs described how they had been generally confident with their initial training but had become disappointed and disillusioned when they arrived at their actual work sites and found that they could not perform as they had been trained 
to perform. Susie complained, "I have to make choices based on where my time is better spent....like do I see this elementary student over a secondary student?"

She was referring to the fact that she had certain younger students who were more likely to benefit from her services than would the secondary students. She said, "I would love to organize groups, you know, according to their needs but there are so many kids with different needs." Susie's worksite did not allow her the time or the flexibility to do her job; instead she had to contend with logistical circumstances, something that she had never encountered in her training sessions.

There was another downside to the SLPs idealized education and training that was noticeably apparent from the data. These SLPs did not do well with the unexpected. Perhaps their training prepared them for ideal situations and not for the complications that regularly arise in a complex and unpredictable school setting. It was as if they had been programmed in another language and could not make sense out of the language that their school jobs required them to have in order to process and perform their duties. The SLPs were aware of just how lacking their education was in light of what practices today's SLPs must be prepared to provide. Many of them believed that it would be impossible for educational institutions to provide members of their profession with a base knowledge of all the practices they were now responsible for providing. The implications of this finding in terms of the type of education that, contrary to their beliefs, can, in fact, inform SLPs is important. And the fact that as a younger graduate Caroline had little knowledge of policy suggests that training protocols 
and universities could do more instructing on the topic of policy dynamics, which will be further discussed in the concluding chapter.

\section{SLPs Navigate Dilemmas with Humor, Idiomatic Expressions, and Solidarity}

Boler's (1999) conception of discomfort is framed as agentive when one bears witness for others. The SLPs shared testimonials that made them feel uncomfortable. Yet with a supportive group who understood their discomfort, they were able to laugh together in a way that validated their understanding that as far-fetched as the comment might be, there was some truth to it...something that warranted some degree of concern. Their monthly subscriptions to the ASHA Leader included listings of ethical violations, and they recognized that their names might someday appear there when there was no other way out of their dilemma but to violate some of the newly established procedures. The focus group provided a platform whereby their colleagues could feel comfortable with their confessions of doubt that should have found a platform within the boards of their professional organizations. Their training validated their appraisal of the situation, but it also made it more uncomfortable for them when it came to finding ways to overcome their problems. To compensate and overcome their problems, the study's SLPs frequently recruited consensus and solidarity with their colleagues with idiomatic "you know" punctuating their speech, especially when discussing serious matters such as ethical adherence and concerns.

The SLPs' humor and jokes about violating ethics codes served two purposes, one of which they may not have necessarily noticed—nor, most likely, would they have wanted to. Bell's (2012) "I's of oppression" defines this as the 
interpersonal phase whereby joke-telling is a device that normalizes oppression. Humor is socially acceptable when one is recognized in a favorable manner rather than an unfavorable one, which is represented in this situation as a code violation. Humor was minimizing the SLPs' acts and could potentially normalize oppression and injustice.

As in the use of humor, imagination is often at play in these situations. SLPs also relied on their imagination when creating solutions to correct the misalignment between their educational experiences and their professional experiences. They discussed how it might be different if the university system offered them more authentic experiences and gave them more control in selecting internships. In so doing, they were ascribing to adult learning theories (Branch \& Frankel, 2016; Izadinia, 2014) and reflective aspects of professional identity formation (Eraut, 2008). SLPs found gaps in their training and they used idiomatic expressions to indicate the seriousness in a light-hearted manner. The messages were well-received, which speaks to the effectiveness of idioms as a persuasive literary device. In this case, SLPs felt ill-prepared in their trainings which they recognized as a compromising factor in the quality of their speech program. On the other hand, while outwardly humor and idioms can diminish the seriousness of the matter in a socially recognizable manner, according to Bell (2012), this also can have serious implications that tend to oppress rather than liberate since it can mask the source of the problems, as was the case with these SLPs. 
A finding of the study was that the SLPs involved had little understanding of the regulations they complained about and the implications they carried for their field in general. For instance, the ideologies surrounding speech and hearing theory did not align with public-school reform sanctions. As revealed in Caroline's figurative explanation, her lesson was out the window and what mattered was an accountability structure. As a result, SLPs are not able to deliver quality services with the broadened scope of practice. SLPs' linguistic and imaginative devices revealed their adaptive capabilities and their resulting uneasiness with issues related to scope of practice and reform initiatives that focused on cost reduction and accountability, i.e., the nine-year rule, state-testing, and Common Core standards.

\section{Entanglement 3: Administration Rewrites of Policy Hinder SLP Work}

Now that Caroline was in an educational setting, her ways of doing things and the ways that she was expected to do them at her job were in conflict. She encountered the problems with neoliberal policy when the expectations of teachers and administration, i.e., teaching to standards and Common Core policies, made her speech lessons less important. According to adult learning theory, knowledge is context bound; participants' agency can be restricted when it is rooted in what is familiar or with what is available (Merriam et al., 2007). Caroline was trained to act according to the methods and models taught by her professors in her university and in her clinical settings. Her job said otherwisewhen her secondary level students were struggling with the rigors of their core 
classes, the focus of her time with them was to teach core class information, not speech therapy.

\section{SLPs Sense Pushback When Working with an Accountability Agenda}

According to Bauman (2009), one can feel powerless when a previously chosen identity is challenged or not appreciated by others. Caroline was introduced to the school expectations and its emphasis on grades through their presence in her job description.

"Our job is to complement what the gen. ed. teacher is doing. For instance, I got a little pushback [from the students] at the beginning, like, 'Oh, you don't give grades, so why would I do what you want me to do instead, if this is due next period?' But a lot of times they were right. Like I couldn't even argue with them."

She seemed angry at first and then confused. She felt forced to work in accordance with factors such as the general education teacher's requests, the department's standards. In other words, her role became helping students pass the course instead of providing necessary speech intervention that, even with her minimal on-the-job experience, Caroline knew was fundamental to the students' academic success.

Although Caroline initially struggled with her teacher relationships as evidenced by her use of the term "pushback," she eventually conceded that "they [the teachers] were right." Gee (2001) explains that to be socially recognized as different can constrain agency; as such, Caroline was enacting a role that a teacher would find acceptable. However. this would not necessarily be the case for 
another SLP with more years of experience, like Ava, who questioned the systems that Caroline seemed to accept. In a relatively short period of time, Caroline had become convinced that the only way she could work in the school was to fit into the system model expected of her. This was clear when she said, "Our job is not to be teaching totally different things but [instead to] allow our students to access the curriculum that's being provided for them. Like we're not here to reinvent the wheel." Caroline aligned with the teacher (the administrative system) and the pressures of meeting common core standards (the regulatory system), another example of the unidentified complexity that an undefined system entanglement creates.

Bauman (2009) states that one should continuously ask what comes next in search of for a robust identity. Likewise, SLPs must search and ask questions as part of their identity formation. Instead of questioning the structure behind the problems, Caroline obliged the teacher's request by convincing herself this practice was legitimate. From the Rhode Island SLPs' perspectives, especially the ones who had more years of experience, the source of the complicated circumstances they found themselves in had arisen simply as a result of two regulatory policies, RTI and the nine-year rule, that in effect, had superseded their past experiences of agency and the ability to provide appropriate treatments. Under the policies of RTI, cases of students with unique needs who were part of the SLPs' large and diverse workloads were often mismanaged when specific treatments, i.e., articulation and fluency, were addressed in intervention groups by non-speech personnel. Ava noted one of the most characteristic complications that 
resulted. "Students are getting re-referred at the middle school." Then she asked in a rhetorical manner, "What are teachers doing that is different? ...What are we doing?" Yet Ava did not blame the system; rather she was frustrated and confused because she did not have a solution or explanation. However, the truth is that Ava's reactions were a product of what happens when schools combine two policies - the federally mandated IEPs and school-wide RTIs - that have separate monitoring methods and criteria for determining speech services.

\section{Mismatch among Policy and Structures Camouflage Problems}

The features of RTI, namely universal monitoring and widespread interventions with research-based evidence, originated with the President's Council of Excellence in Special Education, the Office of Special Education Programs (OSEP), and the National Reading Council (NRC) (Kozleski \& Huber, 2010). The emphasis for early intervention in RTI was based on the NRC's report on minority students (Donovan \& Cross, 2002). These features of RTI were designed to ensure that all students learn using empirically based interventions and certain measures to make this determination; however, these interventions and measures were not focused on students at the secondary level (Fuchs et al., 2010).

According to O'Brien, Stewart, and Mohegan (1995), the structure and culture in the secondary grades limit the feasibility of a responsive and comprehensive literacy model (as cited in Brozo, 2010. Secondary teachers, unlike those in the elementary grades, have students for one hour of blocked instruction (Brozo, 2010). Structural limitations such as block scheduling make 
implementing best practices a challenge. In response to those constraints, SLPs develop patterns of beliefs, thought, speech, and action. Those cultural practices are constituted by values and carry with them normative expectations about how things should be done (Georgiou, Fousiani, Michaelides, Stavrinides 2013). In line with this, one SLP's perceptions of RTI's operations at the secondary level were misunderstood. One elementary grade level SLP, Patrice, had a son with autism at the middle-secondary level, and she reported her observations to the group. Patrice had a double-sided perspective, which carried different values. "Being on both sides of the table, I can tell you as an SLP, and a parent, [that] the high school teacher had no idea what to do with Sam [her son]. They were flying by the seat of their pants... they hadn't compiled any data .... nothing to report out."

In her assessment of her son's middle school teacher and the rest of the staff, Patrice used a metaphor that depicted the staff at this secondary institution as functioning chaotically and ineffectively. Her experience at the elementary level provided her with a model for comparison, and it appeared that her own working experience was more positive-perhaps more communicative and collaborative. Since the staff was the only thing she recognized as different in her experience, it was easier for her to think that the staff was the problem. However, it was the high school operations, i.e., the rotating schedule, lack of planning, and ineffective data-monitoring systems, that were the obstacles to a successful intervention for her son, not the staff. With all these obstacles, it seems quite natural to assume that it would be difficult for her son to transition well to the 
secondary level. However, Patrice did not consider the institutional operations as the problem; she believed that the teachers were not communicating well enough and/or recording the data well enough. Once again, an SLP's assessment of a problem indicated a lack of understanding of the primary contribution of the systems entanglement to the creation and exacerbation of the problem.

\section{Education System Does Not Respond Fairly to All Students}

When speech services are terminated earlier than necessary, SLPs respond less traditionally. Rawls (1971) defines social justice as an original position on which one bases one's perception of society when unaware of one's position or status. Students with unique needs that had formerly been addressed with speech services until the nine-year rule was instituted were now monitored under RTI. Unbeknownst to students, parents, and perhaps teachers, monitoring of students varied and in many cases was inadequate. The SLPs in this study were aware of the inadequacies as they attempted to serve students across grade levels under the rules of two limiting systems, RTI and IEPs. However, SLPs' positioning alongside stakeholders' decreased awareness has had social justice implications for certain students.

While RTI increased SLP student caseloads, the nine-year rule eliminated many students from their rosters who were still in need. As a result of the introduction of RTI in 2004 and the nine-year rule in 2008, two issues came to the forefront that affected SLPs' self-image as professional therapists. First, their caseloads, referred to now as workloads, grew dramatically as a result of the large number of newly identified RTI students who needed services. Four years later, 
the nine-year rule eliminated many nine-year-old students from special education services who had not shown signs of any other issues, a new requirement for continuation of speech services beyond that age. As such, SLPs were conflicted and frustrated on how to help students with special needs.

Patrice commented, "I feel bad for the oral fluency cases that get dropped at age nine. Just because we don't see academic struggles now doesn't mean they won't happen later.” According to Marzano (2004), students who struggle in third grade are more than four times likely to drop-out of high school. Secondary level SLP Ava shared a comment she had made in a discussion about RTI with one of the elementary SLPs in her district. She explained that after they had talked for a while, she had said, "You probably aren't surprised when you hear that your previous students resurface as referrals." Her experience showed her that with the academic rigor at the secondary level, some older students who do not receive the necessary services in elementary school would struggle later in their high school classes. Similarly, Patrice voiced her concerns about the effectiveness of RTI responsiveness for the program's struggling students when she said, "RTI can't fix the students impacted by the nine-year rule ... but there is definitively more that we can do for them." Eddie had another view, "The rule has brought some relief from the large caseloads" to which Kate responded, "Thank God." Oddly, SLPs welcomed a rule which many had admitted was unfair; that act of work relief and consensus had legitimized yet another ill-designed policy.

This comment by both Eddie and Kate brought up the problems associated with increased caseloads. Revisions to the Individuals with Disabilities Act 
(IDEA) 2004 changed the way states determined eligibility of special education services, which in turn directly affected SLPs' caseload types and numbers (Giangreco et al., 2010). In the case of SLPs in this study, the change in eligibility rules created both concern and confusion. For instance, some of the study participants noted that some students reviewed under the monitoring design were being attended to more than others. Patrice said, "You know ....I report out more on the RTI kids and the changes are minimal. We hardly discuss the IEP kids. In fact, my director is calling for a quarterly meeting where we report out on IEP kids." Patrice did note that the director corrected this inequity but explained that by adding another meeting to the SLPs' schedule, he was adding to their already overly heavy workload.

In order to meet the challenges to their students' needs that the interface of these newer rules presented, SLPs often felt obligated to do things quite differently that they would have considered doing in the past, taking on less than conventional pathways within their current job demands in order to remain true to their original professional standards. Patrice, who previously admitted to making a case for another disability, also said:

"I have had to do other things. ...I do lunch groups and give home programs. I collaborate with the teacher when I see an issue with oral fluency and make home programs. The irony is that before the nine-year rule, the typical scenario was the wait-to-fail model ... meaning kids got referred later ... more likely at nine. I can't risk that now. I see my kids earlier and informally, like in lunch groups ... whereas before I did not." 
Patrice sometimes doubted her decisions when she had identified students too early because of the criteria of the nine-year rule. "You know, sometimes these kids are not ready for therapy ... not developmentally appropriate ... [T]hey will not respond to therapy until they are ready. It's too bad things have to be forced." She was not the only one, Kate, in her face-to-face interview, alluded she was seeing students illegally, "under the table". Although they were enacting agency, both Kate and Patrice's imposed dilemmas had legal and ethical connotations in which they were doing more harm than good. For instance, their acts of prescribing therapy that is not age-appropriate could give false impressions of therapeutic effectiveness. She admitted that she did not like that she had to operate this way and that it was certainly not the way she had been trained. On one hand, she created a possibility; on the other, she doubted her decision and felt less sure of her practices.

In the following data, Ava, a secondary SLP, observed a trend concerning referrals in her secondary setting that posed difficult choices because of limited options.

"I can only do what I can do. RTI referrals come late in the third quarter in middle school. The teachers can't help it ... by the time they get to know the kid and make the referral it's December.... Sometimes I get to the kids too late. ... They are failing, unmotivated. When I get to them, I have my schedule organized and the groups that I place them in are not the best. I have no options. I have articulation mixed with language, and they are in different grades ... totally different." 
Like Patrice, Ava felt forced in her choices, having so few options. SLPs typically like to schedule students according to their needs in order to achieve results. However, when they cannot do it that way, interventions are likely to fail and that in itself causes the SLPs to have self-doubts. At the secondary level, SLPs need to have the option to make multiple attempts to address students' speech deficits. When policies and schedules prevent that, SLPs feel frustrated seeing that these students are still struggling and knowing that they should never have been discharged from the IEP services that had explicitly addressed their speech disorder.

From the discussions in this study, it was clear that these SLPs felt great frustration at the way their administrations were failing them and their students. It was also apparent that they were overworked. Their descriptions of how they dealt with these two issues demonstrated that to some degree, their sometimes unconventional and often secretive solutions were perpetuating the problems, even though their intentions, like those of the systems that were causing the problems, appeared to be for the good of their students. Working in secret, the necessary formal, overriding remedies would not likely happen. In effect, their actions were more like short-term reactions.

The complications for SLPs, such as added meetings and late referrals, worsen with system entanglements when the number of intersecting policies begins to accumulate. Increasing complications do not happen by chance - they manifest through complex and dynamic systems, i.e., an ambiguous policy enacted on top of policy that might also have been written in ambiguous language. 
Moreover, these systems operate under the guise of improvement because in general, policymaking is intended to address problems. However, the success of any new policy is first based on the manner in which the policymaking is conducted. This can often be done in ways that are complex and even problematic. According to McGuinn (2015), policies are often expedited to push through agendas. Policymaking that operates this way may look like it is helping and advocating for, in this case, SLPs and their students, thus making the complications that it creates difficult to detect, encounter, and negotiate.

It is not, of course, uncommon for school officials to attempt to address systemic shortcomings by, for example, adding more meeting times. However, when one policy - RTI — intersects with another - IEP — and the parties affected-SLPs, students, parents, and teachers — are not even aware of this intersection, let alone its consequences, solutions like that one enacted are not likely to work. Moreover, the sources of those complications get muddled.

Toniann and Sandy encountered a situation that warranted the need for this level of systemic understanding. Toniann related part of a conversation that she and Sandy had shared previously. At a district-wide speech therapists' meeting, Toniann had commented to Sandy, "Did you notice the name of another school on the eligibility packet?’ Sandy's situationally appropriate sarcastic response was, "Really... like do we look like we have the same conditions." Both SLPs recognized that the other school district was located in an urban area with its own set of needs, so many of which were completely unrelated to those of their school. Yet they were expected to follow the same rules set out by professionals 
who they felt should have known better. Put into the language of systems, the SLPs at one school were expected to adopt protocol on eligibility (established by the federal and state legal systems) that was borrowed from the administration of a school district (education administration system) that had created protocols meant to address the needs of a larger number of students, many with more complex cases, without the funding necessary (education administration system) to provide additional personnel and materials for what this school district lacked to achieve the support level its students needed.

In reviewing the data obtained from the focus group, it was clear that the SLPs had not approached a solution to this dilemma through the lens of system entanglements. Instead they had focused their attention (and their resulting disdain) on the administrator's actions in using a plan from another school that they felt constrained their agency. As they conversed, they discussed the variability among the amount of each district's resources, agreeing that their school did not have the resources that were needed to support the additional personnel (a nurse) or the proper equipment, another aspect that was added to their list of complications. Their conversations did not reveal whether they voted against the protocol, but it was apparent that they did not appreciate the manner in which the administrator had pushed it on them.

\section{SLPs' Feelings and Values Guide Their Improvisations}

These particular SLPs were agentive in addressing certain complications:

1) Common Core and grading took precedence over speech practices; 2) RTI students were given priority over IEP students; 3 ) students with certain disabilities 
were neglected (oral fluency, dysphagia); and 3) the administration lacked resources for meeting the needs of particular students (eligibility protocols/policies). While they are mostly pointing out inadequacies and dissatisfaction, some SLPs responded with less than conventional approaches, making a case for a second disability and perhaps misidentifying students with a developmental delay with a disability. Their discontent was informative and improvisations admirable, but their comments indicated that even with the review of the situation this discussion offered, they were still not gaining insight into the complexity of the variable and intersecting systems that were complicating their work situation.

SLPs may not have been able to explain the intersecting systems; however, they were not passive participants. For example, Sandy’s sarcasm, similar to Goldfarb's (1991) definition of cynicism, was "a form of legitimation through disbelief" (as cited in Kleinman \& Copp, 1993, p. 14). Sandy was perhaps shocked that her administrator chose to distribute another district's eligibility protocol rather devise a district-wide protocol appropriate to the district's needs. Veteran SLPs were often agentive, as exemplified by Toniann, Ava, and Patrice, who improvised to get services. Toniann made a case for another disability, Ava and Toniann worked with students regardless of having a disability or not, and Ava and Toniann questioned entangled systems with exaggerated rhetoric, which is an effective linguistic device that can resist (Holland et. al., 1998; Foss \& Waters, 2007). On the other hand, Caroline, a newly graduated SLP, ultimately complied with accountability protocols when 
she co-taught teacher-centered versus speech-centered lessons (Lipman, 2005). Ava, although more experienced than Caroline, was also constrained when delayed referrals resulted in less than ideal groupings of students. Caroline and Ava aligned themselves with what mattered, what they knew, and what was valued to cope with their perceived dilemmas. In general, SLPs' newer ways of knowing and doing was sometimes contrary to their preferred practices and their feelings indicated they were not passive participants.

\section{Entanglement 4: Policy Make Parent Expectations Virtually Unattainable}

In the case of an education model, system entanglements become more complex and even less transparent for certain stakeholders when the familiar, individual systems operate very differently from one school to another. Since public schools are under federal jurisdiction, one would expect school systems across the country to share similar school policies. However, because of differences in class, socioeconomic status, and race, federal law allows state policymakers to make the necessary interpretations and accommodations. In an ideal world, those changes would primarily be based on those factors referenced above. Instead, many of the states' policymakers are often forced to give preferences to budget and economic concerns over those cultural and societal factors depending on the conditions in the state. Thus, education policies can vastly vary from one state to another, making the work for some SLPs in this country more complicated depending on what state they work in. All these factors, of course, affect the quality of treatment for each state's students. In the 
next section, Susie's accounts of working in two different states reveals how those factors virtually made it impossible for her to meet parents' expectations.

\section{Students' Parents Not Always Actively Involved}

Susie had taught at two very different schools in two different states: one was a suburban school located in nearby New England state and the other was an urban Rhode Island public school. In that out-of-state school, student population was reported as $85 \%$ white, with $70 \%$ of the students achieving high-performing scores in mathematics, with English studies lagging only slightly behind (publicschoolsstat.com). A review of Susie's experiences in each of these schools demonstrated the fact that even within the system entanglements model, there are major differences in how its effects can hinder the work of SLPs when there is a limited understanding of the differences among the stakeholders and with the entanglement of systems in various school settings.

In her comparison of her experiences at the two schools that she worked in, Susie shared the positive aspects of her experiences at the suburban school. She described students, their performances, and the work environment. "There the school was high-performing. We also had more homogenous populations there, fewer complexities in caseload. I had tons of resources and supplies." She went on to say, "I had a busy workload, like here, but the difference is that RTI is higher in proportion to IEP kids."

Although she had only been working in the Rhode Island public school for six months, she had already experienced problems there. Speaking about the Rhode Island school, she observed, "The parents are tough here. I have so many 
who just don't do the homework with their kids." Based on her experiences in her former worksite, she was of the opinion that it was essential that parents take an active role in their child's education in order to maximize student achievement and outcomes - and this included doing homework with their child. When the parents in the Rhode Island school did not live up to her expectations, she became frustrated and the outcomes were less positive, i.e., relationships with parents were constrained. From her observations in this urban school she had not considered other barriers such as single parent households, poverty, and/or second languages. When referring to her suburban school experiences, she had seemed less accusatory of parents. Indeed, socio-economic factors shaped her experiences and the outcomes were either negative or positive.

\section{Regulations do not always take school populations into account. Susie}

did not mention the regulatory policies that served one demographic category over the other. Unbeknownst to Susie, in her suburban school experience, she had been situated in a district that had a good RTI program because they operated under a data system that favored a homogenous population. In that district, students progressed, and staff collaborated. "We had a data-driven system which was well-organized, and everyone had a clear role that was expected, and people came for advice." Not only did the district have the financial capacity to support teachers but it also had a homogenous population that made the work easier regardless of which data system was used.

Populations with more complexities are more challenging because of the overlapping tendency of variables, i.e., comorbid disabilities, poverty, 
socioeconomics, race, and Medicaid eligible. Moreover, the mobility rate, the percentage of students who are likely to move out of district, is higher for urban than suburban students, resulting in knowledge gaps in in the case of the former (Kidcount.org). Policymakers have much to take into account when managing diverse populations; to remedy this on the local level, policymakers there often align with the language of federal mandates, which is problematic for two reasons: first, the language is purposefully left vague and broad (Giangreco et al., 2010); and second, financial incentives are often unacceptable when districts meet them minimally in order to get funding, a case in point being literacy programs (Allington, 2002).

In 1978, Deputy Commissioner of the Bureau of Education Edward Martin was critical of parallel educational systems and carefully created a system referred to as PS 94-142 for exceptional students while respecting the civil rights of minority students. If he viewed the working effects of his system today, Martin would probably be pleased with the equitable focus of RTI for identifying students who need services; but, with a closer look, he might caution policymakers in situations where the fidelity in which RTI is premised becomes diluted when policies intersect and blur the roles between general and special education (Artiles, Bal, \& King-Thorius, 2010).

Clearly, the systems - the educational institutions and political structures of state and local/district policy_are intersecting when the same job in one state is easier to manage than it is in another, a factor that Susie was not accounting for because she took the advantages she had experienced in the suburban school for 
granted. Despite crossing that state border, Susie failed to recognize the systems; rather she implicated the parents. As such, the powers that lie in system entanglements remain invisible and strong. Not apparent to Susie was that demographics, class, and population are part of the institutional and political frameworks, of which she is a part. In her previous job, she had enough resources, unlike her school in Rhode Island, where she had to self-subsidize materials like pens and notebooks. "Look around [in her room]," she said. "Anything with color is my materials." Since working in an urban setting, she was still unsure and not fully accepting of the complications that were surfacing. Without understanding and/or recognizing the factors behind the complications, relying on her suburban experience, Susie operated according to the limitations of what she already knew, and, in that way, her situation seemed to her to be less of a dilemma.

Although public school policy does not work across borders, some SLPs in this study had the impression that policy should work the same, leading them to assume and expect the same of all students and parents, no matter the situation. In their focus groups, many of the SLPs noted that their relationships with parents were strained. But the reality is that parents, like SLPs, were trying to care for the children in the best way possible. Both groups contend with policies and types of institutions that present obstacles to that mission of best care for the students. This would lead one to believe that on the face of it, they should be collaboratively working to change the negative aspects of the policies/systems to improve their outcomes. However, in the model of entangled systems, parents must also be considered as a part of the institutional/political system, which is a view that Susie 
and other SLPs need to understand as well. Parents can, in fact, complicate the learning process because their expectations as well as those of SLPs' stem from unknowingly being part of the structure of the system entanglements because they form one of the systems. In other words, they are part of the complications' roots.

\section{Summary of SLPs' Navigational System}

The complications stemming from dilemmas based on system entanglements are challenging and emotional. In this study, SLPs were guided by three factors, all of which centered on emotions and perceptions. In other words, their perceptions of conflicts between what they knew or valued and what they expected was often misaligned with what was allowed or sanctioned. The state of their emotions was catalytic in that they either drove or inhibited responses. There is little research surrounding the impact of emotions in situations such as this one, mostly because feelings are seldom recorded as data. But in this study, SLPs exhibited an abundance of feelings that were central to their navigation. According to Hochschild (1983),

We experience feelings similar to the sense of hearing and seeing when bodily sensations are joined with what we see or imagine. Like the sense of hearing, emotion conveys communication.....From feeling we can discover our own viewpoints in the world. (as cited in Kleinman \& Copp, 1993, p.7)

Indeed, the SLPs' behaviors in this study were emotionally charged, and the resulting actions, in many cases, led them to enact practices that did not always serve their needs or those of their students. 
Their emotions were reflected in their language and agentive actions. For example, when the SLPs expressed uncertainty in aspects of their practice, their coping mechanisms included dark humor, metaphoric language, and elaborate examples, such as when they Eddie referred to the possibility of SLPs being jailed. Although they knew the topic of ethics to be a serious matter, as professionals, this scenario was believed to be somewhat unlikely, so it was laughable. SLPs joked on the surface about legal ramifications associated with work but beneath their laughter was some real worry and concern. Of note is that the SLPs showed solidarity when they joined in laughter.

This sort of interaction, according to Zembylas (2013), can promote a politics of compassion that is both necessary and valuable for those situated in predicaments involving structural inequalities. Boler (1999) would agree that emotion is a step towards the agentive processes. Comparatively, Mezirow (1997) describes his conception of transformation as a disorienting dilemma that acts in an agentive way (Merriam et al., 2007). As they navigate professional and ethical predicaments, these worried and uncertain SLPs have had to find their agency by improvising and rationalizing other ways of responding and coping (Holland et al., 1998). In this case, they used emotions that casually addressed the seriousness of the matter-joking and laughing — but just as importantly, those emotive comments were socially acceptable and were supported by discursive structures and practices that conveyed a message of solidarity and validation. Their practices were coping mechanisms that served to excuse and/or elevate the SLPs' 
position and, to some extent, transfer responsibilities and implicate others for the complications and dilemmas that they faced.

Besides being nervous or worried, SLPs were simply frustrated or even angry when they perceived that they were not being valued. Dilemmas resulted when their values conflicted with principles sanctioned by authoritative structures, like due process hearings. According to Bell (2012), oppression results from conflicting sanctions principled on regulation, ethics, and economics. In this study, the conflicts led to professional and ethical paradoxes for SLPs. The emotions triggered by some of these sanctioned principles were hurtful. When SLPs' knowledge did not fit the expectations in their worksites, SLP agency was constrained. The SLPs could not do what they were supposed to do, what they desired to do, and/or what they felt was required of them to do for their students. When they had to contend with unsupportive worksites and restrictive regulations, they endorsed what was perceived as valuable. In addition, SLPs felt forced to take actions that were not part of their typical practices. and they were often disappointed with themselves when they had to implement these kinds of practices.

The messages that SLPs hear are received and transmitted through interpersonal situations and institutional systems (Espinoza, 1994). As a result, SLPs absorb ideas about themselves and begin to feel certain emotions, i.e., insecurities, self-doubt, frustration, worthlessness. The internalization of these stereotypes comes from their communities and institutions, i.e., family, college, work. Espinoza (1994) cautions that when one assimilates into a group they can 
also disassociate from their groups and engage in harmful behaviors towards themselves and others. As such, SLPs that exhibit self-deprecating humor and negative feelings towards others, including students' parents, might endorse practices that they would ordinarily not.

Finally, I want to refer to Gee's building tasks in which SLPs' language functioned in navigating dilemmas and shaping identities (2014). SLPs used a variety of idiomatic expressions that connected them to similar oppressive scenarios. Yet, they found new ways of knowing by improvising approaches to circumvent structures that deprived students of services. Other times, they employed rhetorical questioning which is an effective device to stimulate thinking and knowing (Foss \& Waters, 2007). SLPs transmitted beliefs through socially recognizable forms: metaphors, humor, casual language, and sarcasm; all of which had legitimizing effects (Gee, 2011: Bell, 2012 Goldfarb,1991). Other times they used grammatic structures, i.e. pronouns and voice, in locating their positionality that either resisted or endorsed norms. Contrary to their preferred practice and ASHA's mission, they renamed professional as one who follows the law. Despite ethical tensions, their view of professional identity had legitimized a rule that SLPs previously believed was unfair.

\section{Chapter Conclusion}

As SLPs faced the intersecting complications that arose from entangled systems, their previous belief systems were challenged, altering their orientation, all of which subsequently evolved into ethical and professional paradoxes. According to Bell (2012), underlying sanctions within the paradoxes function as a 
mechanism of oppression whereby SLPs consider legalities and economics rather than the ethical/professional implications. Without realizing it, the seeds of oppression, namely system ideologies, are planted, whereby beliefs and practices come to be dominant and others are suppressed. Some of these ideologies were modeled in the SLPs' backgrounds and university training and through SLPs' interactions at those sites as well as their work sites.

As a result, the SLPs had a hand in cultivating ideals and traditions of systems that either valued economics, professional ethics, or legal matters. They were beholden to ideals such as adhering to ASHA's mission, yet they followed their district's protocol regarding RTI procedures. They did so because they were part of a social and political hierarchy that valued RTI protocol over IEPs. Also, because of past experiences and background, these SLPs as well as others valued social recognizability, which in parts of this study translated into obliging authorities, avoiding confrontation and perhaps unemployment, or risking a lessening of their reputation. However, when they felt threatened, their responses translated into other devices that seemed to be more accusatory and retaliatory rather than obligatory.

It is within these school sites that SLPs' interactions and perceptions confirm and transmit certain questionable practices and beliefs. When the study's SLPs made dark jokes and used self-effacing humor, they were not consciously aware of acting or contributing to oppressive conditions. Sometimes, in their witnessing and by standing (Soutu-Manning, 2009), they were aware of injustices, which according to Boler (1999) can be transformative. However, Bell (2012) 
would likely go a few steps further stating that oppression can only be dismantled by redressing ideologies, institutional beliefs and practices, and the system by which those ideologies are transmitted. Unless SLPS follow these findings, they will continue to internalize beliefs that have and will continue to perpetuate other injustices.

In this study, SLPs navigated the dilemmas of these paradoxes through their perceived sanctions, responding with 1) what they knew, 2) what was allowed, 3) what was expected, and 4) how they felt and were recognized. Given their challenging experiences such as due process hearings, exclusions from needed work with students, and an overly heavy workload, they made choices based on how they felt, which meant, for instance, that covertly they no longer went over and beyond in their work or that they felt forced to keep two different documentation records, one for themselves and one to use as in situations where they would need legal protection. They no longer based their responses on what they necessarily knew from their education, training, or past experiences but rather on what the school expected of them based on the current regulations and expectations of other agencies related to their field. Nor did they practice with their perceived level of excellence, which is the standard ASHA expected of them and they expected of themselves.

Yet, even if the face of that last clearly expressed negative set of circumstances, the SLPs defended their actions by attempting to find some amount of agency, sometimes that they themselves questioned as being somewhat deceptive or even deceitful, in order to justify their desire and professional 
obligation to help their students in any way that they could. Carrying this even further, in some cases, SLPs, through their interaction and discussions, recruited others to their viewpoint, one of which was accepting a rule they had termed as "unfair." They did so simply because of their shared experiences, described mostly as negative, and the end result that this rule would lessen their burdensome workload. That sense of relief gave SLPs permission to accept an unfair rule, and in the process become one of its enforcers. As such, SLPs were implicated in the cycle of oppression. 


\section{CHAPTER 6: CONCLUSION}

The purpose of this study was to examine the discourses of Rhode Island's speech-language pathologists (SLPs) working under the state's nine-year rule, which mandated the end of speech and language services for certain students who had reached the age of 9. The SLPs struggled upholding that rule under Response to Intervention which was part of the 2004 revisions to the Individuals with

Disabilities Act (IDEA). In this study, Boler's (1999) discourse on the "Pedagogy of Discomfort," Gee's (2011) writings on familiarity, and Holland's et. al. (1998) assertions about social recognition were essential elements to the SLPs' agency and self-identity in navigating the insurmountable dilemmas that resulted. From interviews with SLPs across the state, I was able to identify certain repeated themes, which I described in Chapter 4 and further analyzed in Chapter 5. Each theme addressed the study's research questions:

(1) What were the challenges and the tensions that SLPs encountered in their work and how did they respond?

(2) What factors did SLPs perceive as significant in the formation of their professional identities?

(3) How did SLPs integrate their background and experiences with the norms of the profession, ASHA standards, and their preferred practices?

\section{Summary of Findings}

All of the data retrieved from the focus groups and the individual interviews determined that the SLPs were part of a systems framework that perpetuated current complications and perhaps created others. At first, like the 
participants in this study, I was not able to identify the roots of the complications; rather I was only able to describe, complain, and sometimes blame, just as they had in the groups. I suspected that policy, namely the nine-year rule, complicated the SLPs' job, which also diminished the SLPs' perceived value, but I also blamed the SLPs for being passive and not resisting.

I needed to revisit policy reform and understand the socio-cultural links between agency and identity and other mechanisms that feed or inhibit power before I could stand back and take a different view. I was able to delve into the power of social recognizability that is part of identity formation and with the help of my advisor acknowledge the power of emotions and their transformative capabilities. I could see how the focus groups had brought these SLPs together in a way that allowed them to feel empowered, while in the same breath be describing a lack of that feeling of empowerment on the job.

Through coding and critical discourse analysis of the SLPs' transcribed interviews, I identified four systems, and within those systems, corresponding complications that posed dilemmas. Speech-language pathologists' professional groups, namely ASHA, the national organization, and RISHA, its state branch, comprised the first of the four systems. In this system, SLP members engaged in processes that included a focused mission and a sharing of values. I identified federal and state policy as the second system, in which groups competed for resources and power. The third system in my study, the education institution, is similar to Bolman and Deal's structural system (2012), whereby change is 
accomplished in a systematic and interactive fashion. Through the people, places, procedures, and protocols in various educational settings, SLPs enacted certain practices and doctrines which shaped their identity. The fourth system was an interface of the other three which is referred to as "system entanglement" and that required SLPs navigation.

The SLP were guided by an emotional needle to orient him or herself on a three-way directional compass: 1) knowledge and training; 2) sanctions-legal, ethical, and economic; and 3) expectations - those of students, parents, staff, administrators, professional organizations, and his or her own. Emotions acted much like a canary in a coal mine signaling something was terribly wrong. SLPs reacted; however, the toxins were barely recognizable.

\section{Chapter Roadmap}

In this chapter, I briefly summarize identified systems alongside the implications, then I offer specific recommendations for SLPs, their profession, and their students. Due to the dynamic and systematic nature of power, SLPs have reached a point in which they have conveyed strong emotions and improvised less traditional responses. They have positioned themselves defensively and offensively, adjusting their positions to barriers in their workplace setting. Therefore, to better position SLPs, I suggest practices within in the context of their work, suggest theoretical approaches to further their knowledge and practices, and lastly recommend future research surrounding their agency. 


\begin{abstract}
ASHA and RISHA
One of the key findings in this study showed that ASHA's broadened scope of practice created complications and resulting dilemmas for SLP. Although ASHA facilitates SLPs' acquisition of knowledge and training, it on the other hand, prescribes a broad scope of practice and standards which are overwhelming and frustrating for SLPs. For instance, SLP's indicated they were fearful about working with students with complex and unique needs because of inadequate training, malpractice risks, and the lack of adequate support. They felt constrained by that scope and believed ASHA does not consistently provide them the advocacy and support they need. Likewise, RISHA, a state organization, was believed to be lacking in their effort against oppressive structures, i.e. the nineyear regulation. Although ASHA and RISHA expect SLPs to uphold a mission and standards of excellence in practice, it seems virtually impossible when SLPs work with minimal resources and supports. In fact, they independently expend energy and money on professional development to find efficient methods to manage their workloads.
\end{abstract}

\title{
Federal and State Policy
}

Overriding policies created dilemmas that had emotional consequences, SLPs were confused, frustrated, and unprepared. These complications mainly focused on policies that mandated cost reduction, accountability, and productivity, all of which shaped SLPs' workloads and practices (Lipman, 2005). These 
complications were rooted in overlapping policies that did not align with the contextual differences. As a result, policy had been written ambiguously which resulted in loose interpretation from state policy makers and school administrators. What follows is confusion whereby SLPs had to sometimes choose to be ethical or professional in managing their workload and best practices or finding a way to manage that was outside some of those parameters.

Policymakers operate under a neoliberal agenda of accountability; that often necessitates evidence, which means implementing another policy. However, in the state of RI there has been no movement towards monitoring the nine-year rule impact. Because there has been no attempt to measure the outcome of the nine-year rule, many SLPs have initiated their own monitoring systems of students and delivered "off the record" interventions. SLPs across the state still struggle with who to serve, how to manage students monitored under RTI, and how to attend to students with IEPs and 504 plans in ways that will guarantee that each student with disabilities receives the opportunity for an equal education.

\section{Education Institution}

This study's analysis of SLPs from two focus groups revealed the relevance of relationships in the education institution and how they take shape in four contexts: places, people, protocols, and procedures. The SLPs' responses toward the quality of these perceived relationships mattered as to the outcomes for the students they served and for their professional identity struggles. The participants shared that their relationships with parents and staff had greatly 
deteriorated because of due process proceedings and intervention protocols. Secondary level SLPs in particular reported that relationships and school procedures and structures were more constrained, which negatively affected their perceptions of their value and significance of their work for their students.

Another point that illustrated the SLPs' awareness of another policy clashing with RTI but not seeing the problem as dually sourced involved the problematic interface between RTI and the nine-year rule. After the introduction of RTI, SLP workloads grew much larger mostly due to the large numbers of RTI students who were now being loosely monitored under a school-specific universal design. As a result, students went either undiagnosed or misdiagnosed, and later, with the rigors at the secondary level, they struggled in their classes and/or dropped out of school. Language and literacy issues are interconnected and persist into adolescent years. This is worrisome given the problematic issues in the education system and the fact that more than eight million students struggle with reading in the fourth grade (Pierre, Grigg \& Donahue, 2005) and those that do are four times more likely to drop out of high school (Marzano, 2004).

\section{System Entanglement}

The majority of the very trying complications and dilemmas that they faced daily arose as a result of the introduction of RTI, then worsened with the nine-year rule. For example, students with unique needs who were part of the SLPs' large and diverse workload were often mismanaged when specific treatments, i.e., articulation and fluency, were not specifically delivered in large 
groups of five to six students. Yet, that situation was the result of having to combine systems - the federally mandated IEPs and school-wide RTIs - that had separate and very different monitoring and exiting criteria. Although the SLPs clearly saw that there were two policies clashing, by citing only RTI as the problem, they failed to recognize the problem as being dually sourced—what this study refers to as system entanglement. Of note, socioeconomic disparities across school districts had created a situation in which SLPs and parents blamed one another when student progress was lagging. Hence, parents and SLPs were implicated in system entanglement.

From this study, it was clear that overlapping policies marginalized certain students. The complications that arose from this example of system entanglement did not happen by chance: they manifested through complex and dynamic systems, i.e., policies. Although these policies created and operated in ways that perhaps were intended to improve the outcomes for SLPs and their students, they did not. In fact, not only did the individual policies create complications but their overlap and faulty interface made the complications more difficult to detect and counter. While policy alone may not be crippling, calamities will arise when a powerful policy partners with other equally powerful laws or rules. Both the policies and the people involved become part of entangled mechanisms of circumstance and relationships in debilitating ways that may or may not have been taken into account earlier on. So even when the individual complications are noticeable, the complex construction of the interface as the 
underlying source is not, which is how power remains mostly invisible and solutions much harder to come by.

\section{What Now for SLPs, the Profession, and Student}

In response to the implications from the systemic structures, the following practices are recommended. In the focus groups, the SLPs had already generated solutions, however, the line between being professional and ethical was something they questioned. In discussing the dilemmas, SLPs were emotive and vocal, those threads are essential to the fabric of change. Therefore, creating agentive spaces is important so that SLPs continue to reflect and evaluate their practices, ethics, and knowledge. To their credit, these recommendations are the product of their improvisations and testimonies.

\section{Practices on behalf of SLP, Profession, and Student}

\section{On the Right Track, Do Not Stop}

Because of the implications for their students, SLPs figured unique ways to serve their students. They coordinated informal groups, and some SLPs decided to collect data on those students and found effective monitoring tools. That data has potential to expose inequities and, if other district SLPs are doing the same, they can compile that data and formally write constituents and their professional organizations about the outcomes. In fact, they can refer to RIDE for open meetings on regulatory matters. They can respond with public commentaries. Their professional organizations RISHA can facilitate work group formations surrounding special interest topics: data monitoring, adolescent literacy, and 
regulatory matters. Of note, SLPs. seemed comfortable talking in groups of four or five to which they agreed the groups were helpful in expressing concerns and understanding them more. That size is important to consider in making workgroups focused and effective.

\section{SLPs Filling the Gaps}

Professional development is necessary for accreditation and filling gaps in knowledge; however, ASHA has a rigorous, and sometimes expensive, process that has to meet certain criteria. RISHA has already developed less expensive ways to gain education hours and collaborated with ASHA for gaining credit through approved workshops that include SLPs conducting specialized workshops. However, RISHA is experiencing declining membership and, for a minimal fee, SLPs can join and strengthen the program that helps them develop professionally and offer a comfortable place to voice their concerns. Novice SLPs would benefit from a professional mentor relationship within that organization. SLPs already provide clinical supervision to student SLPs and clinical fellows; however, an informal, non-graded situation offers a safe place to discuss complications at work. On that note, the process of engagement and empowerment happens, and it can begin at the university level. Perhaps, RISHA can facilitate a process with SLP to collaborate with the university and affiliate with the student professional body. 


\section{Sensing Pushback, Somethings Up}

Recent graduates are working with what they learned in college. For instance, Caroline, a recent graduate, had minimal knowledge regarding policies. That had consequences at her first work site. She quickly learned that teacher's lessons are graded; hence more important. That pressure on teachers meant pressure for SLPs. Caroline recalled sensing pushback from a teacher who had other plans for the co-taught class and Caroline's lesson "went out the window." Caroline reflected in her focus group wishing she knew what she knew now. However, she justified her compliance with "why reinvent the wheel" and that her job was to implement common core alongside the teacher. SLPs have worked diligently in their training and that was not valued in the current school climate. In retrospect, Caroline and the teacher could have had a conversation about their roles and perspectives. In any event, the focus group triggered conversations about the SLP role and the lack of knowledge SLPs have on regulatory matters. According to Toniann, a veteran SLP, her political knowledge was selfdirected, she remained informed by attending meetings at RIDE, working as an adjunct professor, and writing constituents. Caroline mentioned that during student teaching, she was told the reason students were discharged at nine was because of a rule. She did not know if SLPs were addressing that issue with other protocols, she had just accepted it. Education legislation is not sufficiently taught in graduate school nor was it discussed during student practicums. This knowledge is critical to SLPs' work identity and, apparently at the universities, 
that knowledge is lacking. It is imperative SLPs stay informed with inquiries and testimonies as Toniann had done.

SLPs can learn to negotiate pushback. Most of their training includes theory and diagnostic interventions; however, learning about and attending to contextual differences is important. There are many types of interactions which SLPs perform, i.e. SLPs inform parents about their child's disability, they collaborate with teachers and administration, and they conduct professional workshops. They can prepare and rehearse for these typical roles as well as the unpleasant ones, i.e. participating in a due processing hearing. These are skillsets which are rarely practiced but important to agency.

\section{School Hierarchy Matters}

SLPs feel inadequate in their school hierarchy which often impact work with their students. SLPs can counter feelings of alienation with attending educational forums outside of their discipline, co-teaching opportunities, guest speaking at faculty meetings, and delivering workshops for teacher-parent associations. In doing so, they are educating them on the role of the speechlanguage pathologist, speech interventions, and strategies. Less formally, they can volunteer for student field trips and school activities. While this builds relationships with teachers, parents, and students, it has instructional value. Like Toniann, I often observe and interact with students in less structured settings to better assess skills and plan applicable interventions. Context and relationships are extremely important to SLP work and matter to their value and recognition. 


\section{Theoretical and Research Implications}

This study contains theoretical and practical implications for helping current and future SLPs fine-tune knowledge, training, and workplace practices. Most adult learning studies are based on medical professionals - mainly medical students involving best training in patient care (Branch \& Frankel, 2016; Holden, Buck, Sadler, \& Spike, 2016; Eraut, 2008). Those studies highlight the significance of apprenticeships and reflective practices as essential to the medical professional's identity. While speech language pathologists are not classified as medical professionals, there are some significant similarities between the two professions. Like medical professionals, SLPs adhere to a mission in their apprenticeships and training. Medical doctors swear the Hippocratic Oath to "do no harm"; SLPs promise to advocate for their clients and practice excellence in their practices.

\section{Specializations in the Speech-Pathology Profession}

SLPs receive a degree in communicative disorders usually through colleges and universities that offer programs in allied health sciences. In this study, SLPs expressed interest in specialization areas, i.e. dysphagia or literacy. They added physical therapists specialize as well as educators, i.e. science and mathematics. The SLP scope of practice was indeed an area of frustration and perhaps, this study will bring attention to this fact and result in better-work practices and professional identities. To support that possibility, ASHA and university programs should explore work issues related to the scope of practice 
and possibly consider narrowing the broadness with specialization and certification pathways.

\section{Professional Reflection and Community Practice, a Means to Agency}

Farrell (2011) asserts reflection is one of the central components related to the concept of self as it brings tacit conceptualization to a level of awareness (as cited in Izadinia, 2014, p. 435). Although SLPs had previously described the roots of their complications they were not thoroughly understood. Research has identified the importance of community of practice (COPs)(Murray, 2008; Poyas \& Smith, 2007) through reflective activities (Dinklemann, 2011) and shared education and professional experiences (Hockings, et al, 2009). In the focus groups, SLPs did their best to contend with complications resulting from entangled systemic forces but did not understand that their situation did not arise from the failure of one rule or one legal proceeding. Instead, they targeted parents, ASHA's agenda of scope, new policy, lack of administrative leadership and support. The participants' agency depended on their awareness of the different factors that ultimately contributed to their identity (Izadinia, 2014). In the focus groups, the SLPs benefitted from opportunities to discuss and respond to problems they faced.

\section{A Human Frame for Managing Complications}

One way that the SLPs in this study might have addressed their issues would have been by honoring one frame of Bolman and Deal's (2013) organizational system: the human resource frame. That frame includes the 
qualities of recognition, significance, solidarity, and feelings, all of which are important components of one's sense of value. In this study, the human resource frame, which focuses on team building, professional development and well-being, was underutilized. According to Hargis \& Bradley (2011), employee satisfaction, relationships, and commitment are important to effective and sustainable work practices. Perhaps instituting a human resource frame within a community of practice would hold promise for the restoration of these SLPs' sense of professional identity and navigation of oppressive dilemmas.

\section{Future Research}

This study's theoretical frame and methodological approach primarily identified and explained factors that contributed to the formation of SLPs' agency and how that shaped their professional identity while working under the regulatory nature of the overriding education policies. However, it was clearly evident from the discussion points that the women members of these groups especially felt that their value, both professionally and personally, was diminished. Perhaps they needed more recognition and reciprocity, which, according to Belenkey et. al (1997), are important factors in women's way of knowing in particular. Although this was not a focus of this study, it is likely that a future study that explored this phenomenon of gender differences in the speechlanguage profession would be worthwhile.

Through their discussions, I learned about the SLPs' identities and I got the sense they wanted to talk more and were interested in a future study. They 
enjoyed meeting at different locations and sharing stories comfortably while eating pizza or having coffee. Outside of their institutions, they were uninhibited, vocal, and supportive. However, SLPs are typically alone in their settings, assigned to one school. As such, I would want to explore the SLPs through their discursive and social linguistic practice but within their institution. Perhaps, I could observe them in various roles, co-teaching, conducting workshops, and in district wide SLP meetings. By using this context, I can better describe and explore agentive capabilities and opportunities which they referred to in the focus groups and ascertain differences between the veteran and novice SLPs.

\section{Limitations}

To enhance the validity of the findings, qualitative research focuses on trustworthiness through dependability, credibility, confirmability, and transferability (Guba \& Lincoln, 1998). Credibility refers to the study's account of the array of thematic patterns in data; transferability refers to the study's account of the situational uniqueness of the study's context; dependability refers to the study's account of instrumental changes; confirmability refers to the study's account of investigator predilections (Guba \& Lincoln, 1998).

To accomplish credibility, I took initiatives such as securing and protecting data sources so that the SLPs could tell their stories as thoroughly and completely as possible. Also, the interview locations were carefully selected so that SLPs could comfortably and privately tell their stories. I conducted member checks via email correspondence. In this manner, I was able to obtain dependable 
and credible data. The dependability of data was achieved through analytic memos and categorical logs in Saturate, a software application. The confirmability of that data was achieved numerous ways: first, I utilized multiple audio devices and second, those recordings were transcribed manually and with an outside service; and finally, I met frequently with a critical friend, my major professor, to confirm observations and reflections with data findings. Transferability was accomplished with the combined application of descriptive coding and critical discourse analysis of the SLPs' transcribed interviews. In terms of acknowledging my subjectivities and influences, I kept a journal in which I noted questions for myself and my advisor. In retrospect, I found that meetings with my advisor were crucial in controlling any biases.

In terms of improvements, a larger and broader sample of participants would have strengthened the findings and perhaps added more insight. In terms of this study, I had difficulty obtaining a purposeful sample but fortunately was able to recruit participants with varied work experiences in elementary and secondary level schools. The majority of participants had more in common rather than less, i.e., years of experience, gender, and geographic region of school locale. Data was abundantly available about the lives of women SLPs with years of experience working in rural-fringe and suburban school settings. I encountered a problem when one of the focus groups exited the study. In its place, I substituted a group from a different school district in which the demographics and geographic locale were slightly different (rural fringe versus suburb) and at which I was an 
employee. My familiarity with that group's SLPs was initially a concern; however, that factor ended up contributing to their comfort in sharing stories. Also, I discovered that I was presumptuous in thinking that I "knew" their stories, which in fact turned out to be distinctly different and informative. Overall, my study and its theoretical frames confirmed the fact that one's background and experiences are important to one's agentive capabilities as well as the communal act of sharing stories.

One more point on trustworthiness. I would be remiss if I did not mention the value of my pilot study in which I procured a priori themes. The outcomes of that study informed the theoretical views and the methodology for this study, all of which added to its trustworthiness.

\section{Conclusion}

The results of this study showed that sanctions created paradoxes whereby the SLPs were conflicted about how to do their work with integrity in the face of so many obstructions. They discussed the legal ramifications of the due process hearings in which parents were led to believe that they should expect more services for their child and often got them, which perforce altered the quality of services the SLPs offered to their students. Other times they acknowledged that productivity demands, and increased workload numbers changed their practices. More problematic, as seen from this researcher's perspective, was the fact was that several of the SLPs welcomed the presence of a rule that they also declared to be unfair and deleterious for their young clients simply because viewed through a 
different lens, it relieved their workload numbers. Their stories detailed their feelings of frustration and the lack of support they encountered without a clear sense of how to "fix" it. Sometimes, their only viable responses were to make a case for an as yet undocumented disability in order to secure services they knew would become necessary or, lacking enough available time in their word load, to choose to treat one child over another. Their stories indicated that they were indeed conflicted given the complexity of a three-way directional system in which an emotional needle functioned as a signal.

They contended with challenging issues that arose from their constant interface with three individual systems and the resulting entanglements among these systems. The complications and dilemmas that arose from these issues were difficult to see, much less overcome. The "system" that was for the most part missing from all the SLPs worksite situations was one that emphasizes human needs and connection and that, according to Bolman and Deal (2013), is important to agency in an organization. In its place were the messages that SLPs heard came from due process hearings and interactions with disgruntled parents and frustrated teachers. Their perceptions of those relationships factored into their selfassessments, triggering emotional responses that obstructed rather than revealed possibilities for change.

As the systems became entangled, the complications became less discernible, making attempts to navigate them more difficult. The participants in this study worked with what was at their disposal. As a result, the outcomes were 
not always in the students' best interests, which contradicted the SLPs' mission of excellence in practice and advocacy for their clients. As I began to discover and understand the systems and their entanglements that were at the root of the problems, the true dynamics of power were unveiled. By framing the tensions and complications as systems that upon intersection became more challenging to understand and therefore more resistant to change - and in some cases, more susceptible to further complications - I began to grasp how the overriding systems challenged the SLPs' own belief systems and values, and why their response was often to seek unorthodox ways to solve their dilemmas. 
ERLID \# 5022 Time Schedule

ERI IDA som

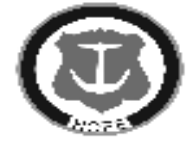

State of Rhode Island and Providenca Plan;ations

Department of State I Office of the Secretary of State

Nellie M. Gorbea, Secretsry of State

Rules and Regulations Fling Form

L. A sency Narne and Address

Edueation, Board of Regents for Elementary and Secondary

255 Westminster Street

2. Titk of Rule

Regulations Governing the Education of Children with Disabilities

3. Statutory Souras of A w thority

R.LG.L. 16-24-2

4. Consies Explanatory Statement - 842-35-2.3.

These regulations are promulgated to generally conform to applicable federal statutes and regulations governing the education of children with disabilities

5 Tros- of Eiling

\begin{tabular}{|c|c|}
\hline \multicolumn{2}{|c|}{ Emergency Rules } \\
\hline $\begin{array}{l}\text { A. Emergency } 120 \text {-day initial - \$4:-32-35-3(b) } \\
\text { Adoption } \\
\square \text { Amendiment of ERL.ID: } \\
\text { Repeal of ERL.ID: }\end{array}$ & $\begin{array}{l}\text { A2 Emergency 90-day menewal - } \$ 42-35-3(\mathrm{~b}) \\
\text { E Adoption } \\
\text { Amendment } \\
\text { Indicale ERI.ID of } 120 \text {-day initial }\end{array}$ \\
\hline
\end{tabular}

Brief Statement of Reason for Finding Imminent Ftril ş42-35-3(b)(2):

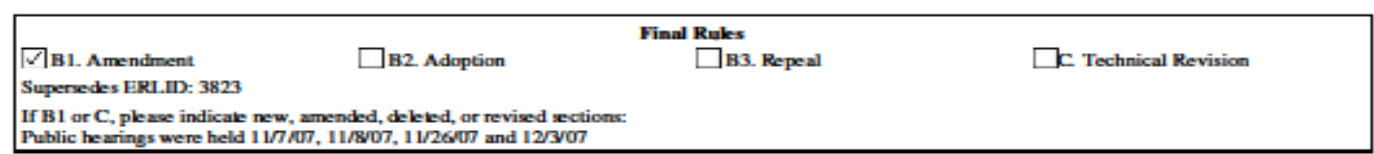

6. Notice and Hearing Information

Date of Public Notice - s/22-35-3(a)(1): 11 /19/2007

Date of Public Hearing - \$42-35-3(a)(2): $12 / 03 / 2007$

End of Comment Period: 12/14/200?

Z.Asency Additional Information - Web Page

hatpoll

8. Certification

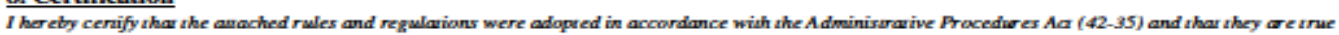
copies of ahis Deparmens, artess

Name

Tilk
Notary Public

Subscriled and sworn before me

this _any of 


\section{Appendix B}

ERLID \# 6746 Clerical Error and Minutes Link

http://sos.ri.gov/documents/publicinfo/omdocs/minutes/316/2010/19779.pdf

ERI ID: 646

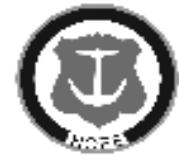

State of Rhode Island and Providenca Plan;ations

Department of State I Office of the Secretary of State

Nellie M. Gorbea, Secretary of State

Rules and Regulations Fling Form

1. Asency Name and Address

Education, Board of Regents for Ekementary and Secondary

255 Westminster Street

2 Titk a Rule

Regulations Governing the Eiducation of Children with Disabilities

3. Statutary Sounce of A nthority

R.LG.L. 16-24-2

4. Concise Fxplanatory Statement- 842-35-23

When the 2010 Regulations (ERI.ID.6121) were filed there was a clerical oversight on the Title of the Regulation - It should read - Regulations Goveming the Education of Children with Disabilities

5.Tros of Eriling

Emergency Rules

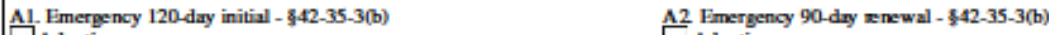

Adoption

$\square$ Repal of ERI.ID:

EAmendment

Brief Statement of Reason for Finding Imminent Feril \$̧42-35-3(b)(2):

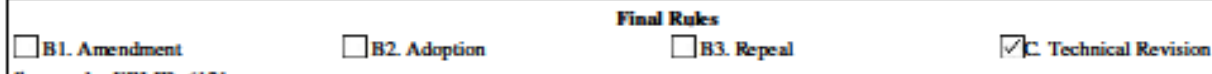

Supersedes ERL.D: 6121

If B1 or C, please indicak new, amended, delekd, or revised sctions:

Title Change - It should reat Regulations Governing the Education of Children with Disabilities.

6. Notice and Hearing Information

Date of Public Notice - 8⿱ $42-35-3(\mathrm{a})(1)$ :Notice Not Required

Date of Public Hearing - \$42-35-3/a)(2):Hearing Not Required

End of Comment Period:

Z. A sency Additional Information - Web Page

hitp:ll

\section{Certification}

1 her eby cerrify that the awached rules and regularions were adopted in accordance with the Adminisuraive Procedies Aa (42-35) and thar they are true copies of this Deparmenr, artes,

Name

Tilk
Notary Public

Subscriked and sworn before me

this dry of

ERI ID: 646 


\section{Appendix C}

\section{ERLID \# 6693 Evidence of errors prior to 2010 filing}

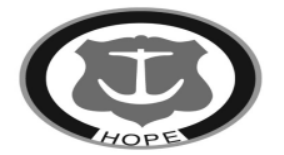

State of Rhode Island and Providence Plantations

Department of State I Office of the Secretary of State

Nellie M. Gorbea, Secretary of State

(Repealed) ERLID\#: 6693

\section{Rules and Regulations Filing Form}

1. Agency Name and Address

Education, Board of Regents for Elementary and Secondary

255 Westminster Street

\section{Title of Rule}

Regulations Governing the Education of Children with Disabilities

\section{Statutory Source of Authority}

R.I.G.L. 16-24-2

\section{Concise Explanatory Statement - \$42-35-2.3}

The 2008 regulations are being repealed in order to correct an inadvertent clerical oversight when subsequent regulations were posted in 2010. The 2008 regulations (ERLID:5022) were not superseded when the 2010 regulations (ERLID:6121) were filed.

\section{Type of Filing}

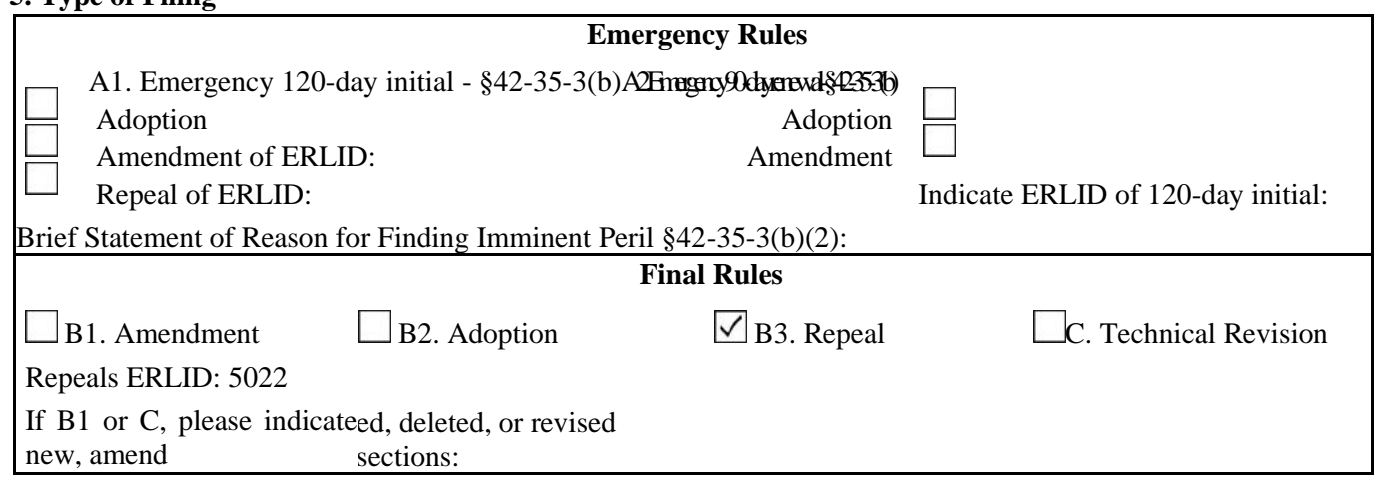

\section{Notice and Hearing Information}

Date of Public Notice - §42-35-3(a)(1):11/18/2011

Date of Public Hearing - §42-35-3(a)(2): Hearing Not Required

End of Comment Period:12/19/2011.7. Agency Additional Information - Web Page

\section{Certification}

I hereby certify that the attached rules and regulations were adopted in accordance with the Administrative Procedures Act (42-35) and that they are true copies of this Department, attest,

\section{Name}

Title
Notary Public

Subscribed and sworn before me

this___ day 


\section{Appendix D}

Participants/Focus Groups and Work Aspects/Characteristics

\begin{tabular}{|l|c|c|c|c|l|l|}
\hline N=9 SLPs & Age & $\begin{array}{c}\text { Years } \\
\text { Working }\end{array}$ & $\begin{array}{c}\text { FG 1 } \\
\text { RISHA }\end{array}$ & $\begin{array}{l}\text { FG 2 } \\
\text { MS }\end{array}$ & Level & Region \\
\hline Eddie & 47 & 10 & $X$ & & K-12 & Urban \\
\hline Tim & 58 & 32 & $X$ & & Middle/High & Urban \\
\hline Toniann & 59 & 33 & & $X$ & Middle/High & $\begin{array}{l}\text { Rural } \\
\text { fringe }\end{array}$ \\
\hline Sandy & 47 & 21 & & $X$ & Middle/High & $\begin{array}{l}\text { Rural } \\
\text { fringe }\end{array}$ \\
\hline Caroline & 25 & 1 & & $X$ & Middle/High & $\begin{array}{l}\text { Rural } \\
\text { fringe }\end{array}$ \\
\hline Ava & 43 & 16 & & $X$ & Middle/High & $\begin{array}{l}\text { Rural } \\
\text { fringe }\end{array}$ \\
\hline Susie & 36 & 9 & $X$ & & K-6 & Urban \\
\hline Patrice & 46 & 21 & $X$ & & K-6 & $\begin{array}{l}\text { Rural } \\
\text { fringe }\end{array}$ \\
\hline Kate & 36 & 13 & $X$ & & K-6 & Suburban \\
\hline
\end{tabular}

\begin{tabular}{|c|c|c|c|c|}
\hline$N=9 S L P S$ & $\begin{array}{l}\text { Elementary } \\
\text { IEP and } \\
\text { RTI }\end{array}$ & $\begin{array}{l}\text { Middle * } \\
\text { IEP and } \\
\text { RTI }\end{array}$ & $\begin{array}{l}\text { Secondary * } \\
\text { IEP and RTI }\end{array}$ & $\begin{array}{l}\text { *Middle/High } \\
\text { Workload }\end{array}$ \\
\hline Urban $=2$ & 0 & 38 & 15 & 60 \\
\hline Suburban=3 & 32 & 25 & 12 & 45 \\
\hline $\begin{array}{l}\text { Rural } \\
\text { Fringe }=4\end{array}$ & 0 & 27 & 16 & 48 \\
\hline
\end{tabular}


Appendix E

\section{CONSENT DOCUMENT and IRB Approval}

\section{Rhode Island College}

A Qualitative Critical Discourse Analysis of RI Speech-Language Pathologists' Social and Linguistic Practices: An examination of their professional identities contending with federal and state education policy

You are being asked to participate in a research study about professional identity formation for speech language pathologists in the context of educational policy. You are being asked because you have been contending with policy which impacts how you manage and deliver speech-language services in elementary and secondary schools. Please read this form and ask any questions that you have before choosing whether to be in the study.

Patricia Murtagh, a doctoral student at Rhode Island College, is doing this study under the supervision of Dr. Janet Johnson, her dissertation advisor.

\section{Why this Study is Being Done (Purpose)}

We are doing this study to learn how speech-language pathologists (SLPs) integrate context and personal influences with standards of the speech-language profession in forming a professional identity. We are also looking at what sorts of opportunities and/or possibilities result when they contend with policy implementation.

\section{What You Will Have to Do (Procedures)}

If you choose to be in the study, we will ask you to:

1. First, you will read and answer some survey questions. The questions ask basic things about yourself like your age, your highest degree, how many years you have worked at your school site, where your school is located, and whether your school is an elementary or secondary school. This will take about 10 minutes. If you consent to participate in the study, you will be asked to do the following:

- Participate in a focus group of other SLP's, conducted by me, Patricia. There will be two focus groups contextualized by their membership/institution. You will know which group you are by referencing the subject area in this email. One focus group has only 4 SLPs available in its membership. The other group's participants are diverse and part of a larger membership; therefore, its number depends how many consent to the study and the differences in their background. 
We will talk and answer questions about professional work, education, policy, and anything else that comes up about working as a school SLP. This session will last approximately one hour and will be conducted at an off-campus site. Using a neutral site provides some confidentiality so that you can speak freely and openly. The Warwick library has multiple sound-proof rooms available. This session will be audio-taped and you will use assigned pseudonyms when addressing each other. To remain mindful of privacy precautions, you will wear name tags with pseudonyms.

- Participate in an individual face to face interview, again with me, Patricia. In this interview, I will ask about your personal background and influences which led to this profession. I will also ask about work relationships, your feelings about implementing educational policies, and your feelings about professional organizations for SLPs. Also, questions stemming from the focus group may emerge. This session will be audio-taped and I will interview you using the assigned pseudonym. Your time is important and valuable; therefore, you can choose a time and place convenient for the interview.

\section{You Will Be Recognized (Compensation)}

As a way to thank you for your time, we will offer refreshments and beverages at the focus group with take-away bags containing speech tools. You will also participate in a raffle where half of the members will be eligible to win gift cards each valued at $\$ 25.00$. Those cards will be issued at the end of the study. If you stop, we will pay you $\$ 5.00$ for the online survey and $\$ 10$ for answering questions in the focus group.

\section{Risks or Discomforts}

Although the risks are minimal, you may find that answering some questions is unsettling. If this is the case, you can contact persons in your institution. For focus group \#1, John Magner, the Director of North West Special Education Collaborative, may be contacted at 401-674-4106. For focus group \# 2, Mary Boyle, the President of the Rhode Island Speech-Language Hearing Association (RISHA) may be contacted, her email is mboyle@nssk12.org. We think these questions and answers would be similar to the kinds of things you talk about with family and friends. You can skip any questions you don't want to answer, and you can stop the interview at any time. You may withdraw from the study at any time.

\section{Benefits of Being in the Study}

Being in this study will not benefit you directly.

\section{Deciding Whether to Be in the Study}

Being in the study is your choice to make. Nobody can force you to be in the 
study. You can choose not to be in the study, and nobody will hold it against you. You can change your mind and quit the study at any time, and you do not have to give a reason. If you decide to quit later, nobody will hold it against you.

\section{How Your Information will be Protected}

Because this is a research study, results will be summarized across all participants and shared in reports that we publish and presentations that we give. Your name will not be used in any reports. We will take several steps to protect the information you give us so that you cannot be identified. Instead of using your name, a pseudonym will be used. The information will be locked with password and kept in a locked office, and seen only by the researchers. The only time I would have to share information from the study is if it is subpoenaed by a court, or if you are suspected of harming yourself or others, then I would have to report it to the appropriate authorities. Also, if there are problems with the study, the records may be viewed by the Rhode Island College review board responsible for protecting the rights and safety of people who participate in research. The information will be kept for a minimum of three years after the study is over, after which it will be destroyed.

\section{Who to Contact}

You can ask any questions you have now. If you have any questions later, you can contact Patricia Murtagh at patriciamurtagh8@ gmail.com (401) 954-3827 and Janet Johnson, major advisor at Rhode Island College at jjohnson@ ric.edu (401) 456-8018.

If you think you were treated badly in this study, have complaints, or would like to talk to someone other than the researcher about your rights or safety as a research participant, please contact Cindy Padula at IRB@ ric.edu, by phone at 401-456-9720.

You will be given a copy of this form to keep.

\section{Statement of Consent}

I have read and understand the information above. I am choosing to be in the study "Rhode Island Speech-Language Pathologists' Social and Linguistic Practices: A critical examination of their professional identities contending with federal and state education policy." I can change my mind and quit at any time, and I don't have to give a reason. I have been given answers to the questions I asked, or I will contact the researcher with any questions that come up later. I am at least 18 years of age.

I __agree __ do not agree to be audio-taped for this study. 
Print Name of Participant:

Signature of Participant:

Date:

Rhode Island College Institutional Review Board Approval \#: 1617-1447

Expiration date: $3 / 21 / 20$

Name of Researcher Obtaining Consent: 
Appendix $F$

Regulation Review

\begin{tabular}{|l|l|l|l|}
\hline ERLID \# & Date & Function & Notes \\
\hline 5022 & $12 / 14 / 2007$ & $\begin{array}{l}\text { Eligibility rule } \\
\text { filed }\end{array}$ & $\begin{array}{l}\text { Hearings held before } \\
\text { announced }\end{array}$ \\
\hline 5749 & $05 / 08 / 2009$ & $\begin{array}{l}\text { Adoption of SE } \\
\text { eligibility }\end{array}$ & $\begin{array}{l}\text { Hearings held after } \\
\text { announced }\end{array}$ \\
\hline 6121 & $06 / 03 / 2010$ & $\begin{array}{l}\text { Adoption of SE } \\
\text { eligibility }\end{array}$ & $\begin{array}{l}\text { Hearings held before } \\
\text { announced }\end{array}$ \\
\hline 6693 & $12 / 19 / 2011$ & $\begin{array}{l}\text { Repeal 2010 } \\
\text { rule }\end{array}$ & $\begin{array}{l}\text { Clerical errors in filing } \\
\text { resulted repeal }\end{array}$ \\
\hline 6746 & No date & $\begin{array}{l}\text { Supersedes 6121 } \\
\text { A title error in 6121 } \\
\text { resulted in this } \\
\text { revision }\end{array}$ \\
\hline 7377 & $08 / 17 / 2013$ & $\begin{array}{l}\text { Amendment } \\
\text { addressing } \\
\text { protocols for } \\
\text { students w/ } \\
\text { Medicaid }\end{array}$ & $\begin{array}{l}\text { Supersedes 6746-No } \\
\text { hearing needed }\end{array}$ \\
\hline
\end{tabular}




\section{Appendix $G$}

Interview Questions

\section{Groups}

Topic One: Personal and Professional Responses to the Nine-Year Rule

Leadoff: In 2007 a special education regulation was approved in which a rule called the Nine-Year Rule was enforced in the fall of 2008. How do you feel about the NineYear rule and the changes that it poses for you, your students, their parents, and the teachers?

(implicit categories: How do those changes in practices/programs compare with the standards set by the American Speech-Language and Hearing Association (ASHA), your knowledge and training, and the programs/practices before 2008?)

\section{Possible follow-up questions:}

- Specifically, what does that rule change in your practices AND with whom you work; for instance, how do you work and other people work now as opposed to before, what roles overlap, what previous jobs are distributed or shared? How's that working?

a. Describe what an SLP workday looks like operating under the Rule alongside RTI, and how it is different than before the Rule was passed. (Think of protocols, meetings, conversations, feelings)

- How do your caseloads, treatment plans, and delivery of services differ (as a result of the Nine-Year Rule)? (Think of your scheduling, systems in place, the way you collaborate and consult, or how others work with you.... referrals, are they timely, or other programs, less informal, data processes? [Even CASEL SEL learning imposed, who will make standards, what data are they collecting, who ultimately decides]

a. In terms of other changes, what other trends/patterns have you noticed? Are there shifts in the types of student you see? Where are those students now and how are they doing?

b. If you work in more than one school how does your job/caseload differ under that school's operating systems/supports/resources i.e. RTI, leadership, team work?

c. How have changes required by the Rule aligned with what you were taught in your professional training, and with ASHA and RISHA mission statements? If terms of schooling, how did you acquire knowledge on SE legislation? How practical was it? / What did you get from it? How do you remain current? Do you know what their teaching now, i.e. 
policy, and content, topics/electives?

d. How has the Rule changed your belief systems and training about best practices? [data and empirical]

- How did the above changes impact relationships with students, parents, teachers, and administrators? Also, if you have been a clinical supervisor, please address how this has changed that relationship?

- FACT about those we serve: SLI is the second largest disability what does that mean now in terms of the rule and for those impacted by it? SLI and SSD encompasses issues with different etiologies, consider the sub-categories or other issues for those students. Who and why are they most vulnerable?

- For those of us who worked in 2007-2008, reflect on the passing of the rule, your involvement, and some of the ways your district implemented it? a.With that rules' passing as well the revisions in IDEA's 2004/2006, what is your schools' stance/protocol for determining SLD identification? How does RTI and the NineYear Rule align within these protocols? Think of some of the detriments or benefits, how do you see your role being better utilized?

\section{Topic Two: Resources and Support for SLPS}

- Leadoff: Some of you are at the secondary level, and some are geographically situated elsewhere. Also, we know, based on SES and population, monies are different for school systems. Given some of these reasons, you and your schools may have less autonomy, flexibility, and/or access to more/less resources, tell me, how do those resources impact RTI implementation alongside the rule? How does it impact your job? Now think of those students you suspect, or know were terminated at age 9, What's systems/programs are in place for them and how is it working?

You have implemented this rule for almost eight years. How have you or your school resolved or worked out conflicts/issues associated with the rule?

(implicit categories: What kinds of resources, i.e. at the school and district level and/or at the state level and/or national level, have helped with implementing the rule?)

\section{Possible follow-up questions:}

- Where/who do you, RI public-school based SLPs, go to when you have concerns and/or need help with their practices/settings? How are these requests received?

\section{READ THE ASHA STATEMENT}

- As credentialed SLPs, WE stand by an ASHA code of conduct AND a mission statement, "we pledge excellence in advocacy and practice" recommended by the American Speech-Language and Hearing Association 
(ASHA). Please describe that means and how you believe you are upholding that pledge..

- Describe your understanding of ASHA's views on these changes. How has ASHA and RISHA helped? How else can they help?

- How are universities planning and aligning with the realities of today's practices and policies?

- What is your vision for this field? THINK: GLOBAL Face-to-Face Semi-Structured Interview Questions

Topic One: Personal History/Background

Leadoff: How do certain events and people from your past, negative and positive, impact the decisions you make and the way you perform your SLP role?

(implicit categories: How do certain people and events compare in making a difference for the way you practice?)

\section{Possible follow-up questions:}

- What sorts of experiences are detrimental to the development of the SLPs?

- Looking back, what persons and/or events influenced you in being/becoming an SLP?

- What kinds of supports helped in becoming a SLP? What were the obstacles?

- What kinds of tensions and challenges have you encountered in becoming an SLP and how have they prepared you for this profession?

\section{Topic Two: Current Work Conditions and Personal Relationships}

Leadoff: How do personal relationships, your perceptions, and conditions, i.e. school locale and setting, caseload, impact your decisions, practices, performance, and students' future/progress?

(implicit categories: How do your perceptions, i.e. of your job performance and what you think others think about you, compare in how they influence your work with students. Also, how do conditions, relationships, and perception compare in terms of impacting the work you do?)

\section{Possible follow-up questions:}

- Describe the circumstances and outcomes when you made a decision that conflicted with your professional or personal ethics and/or with others around you.

- How do you think parents perceive your performance in terms of how you enforce and manage the Nine-Year Rule alongside other policies/programs, i.e., RTI or 504 plans?

- How do you feel about your performance in terms of implementing the rule?

- How do you think students terminated or impacted by this rule are doing? What evidence do you have for this?

- During these changes, how do you think you have managed working relationships, collaboration with teachers/parents, and in advocating for students? 


\section{Topic Three: Overall Effects on Self-Efficacy as an SLP}

Lead-off: What sorts of things can you think of that would help you do your job the way you would like?

(implicit categories: What are some opportunities and possibilities in the SLP

profession? Elaborate by considering what you have experienced, what you know, and what you can imagine?)

- Given a second chance, what would you change/keep in terms of career path and/or education pursuits?

- What do you envision in your future in terms of working in this profession? 


\section{Bibliography}

Adams, M (1990). Beginning to Read: Thinking about Print. Cambridge Press: MIT.

Adler, M \& Van Doren, C. (1972). How to Read A Book. New York: Simon \& Schuster.

American Disability Act (ADA) (2017), Revisions to ADA: interventions and accommodations is mitigating. Retrieved on March 3, 2017 from http://www.adainfo.org/sites/default/files/ADA-Overview2017Update-1slide-per-page-handout.pdf

Allen, Ukrainetz, Carswell, 2012. The narrative language of three at risk first graders. https://doi.org/10.1044/0161-1461(2011/11-0024)

Allington, R. (2002). Big Brother and the National Reading Curriculum. How Ideology Trumped Evidence. Portsmouth, NH: Heinemann.

Al-Mondhiry, R. (2008). Rhode Island to limit speech-language treatment: State association oppose age limits that will affect children with disabilities. ASHA Leader. Vol 13, 7-8.

Apel, K. \& Henbest, V.S. (2015). Affix meaning knowledge in first through third grade students. Language, Speech, and Hearing Services in Schools, (47), 148-156.

Artiles, A. J., Bal, A. \& King-Thorius, K. A. (2010). Back to future: A critique response to response to intervention's social justice views. Theory into Practice, 49 (4), 250-257.

ASHA surveys (2000, 2004, 2008, 2010, 2011 \& 2014). Retrieved on March 1,2019 from https://www.asha.org/uploadedFiles/2018Schools-Survey-Summary-Report.pdf

ASHA Survey on Special RI Regulation. Retrieved from sadams@asha.org on March 12, 2016.

Bauman, Z. (2009). Identity in a globalizing world. In Identity in Question. Ed. London: Sage.

Beck, A. \& Dennis, M. (1997). Classroom-based Interventions. Language Speech-Hearing and Hearing Services, (28), 1997. 
Beecher, C.C. (2010). Response to intervention: a socio-cultural perspective of the RTI problems and possibilities. Journal of Education, 191, 1-7.

Belenky, M., Clinchy, B., Goldberger, N. \& Tarule, J (1997). Women's Way of Knowing: The Development of Self, Voice, Mind. BasicBook Publishing New York.

Bell, J. (2012). The Four "I's” of Oppression. Somerville: YouthBuild, USAPrint.

Bellon, Vereen, \& Ogletree, 2001 A study of a service delivery model in rural northwestern North Carolina with parent surveys and interviews with speech-language pathologists. Contemporary Issues in Communication Science and Disorders, 28,123-132

Bishop, D. V., Adams, C. A. (1990). A prospective study of the Relationship between specific language impairment, phonological disorders and reading retardation. Journal of Child Psychology and Psychiatry. 31, 1027-1050.

Blackburn, M.V. (2003). Talking together for change: Examining positioning between teachers queer youth. In J.A. Valdeboncoeur \& amp; L.P. Stevens (Eds.). Reconstructuring "the adolescent": Sign, symbol, and body, 249-270. New York, NY. Peter Lang.

Bodman, S., Taylor, S., \& Morris, H. (2012). Politics, policy professional identity.English Teaching: Critique and Practice. Vol 11, 14-25.

Boler, M. (1999). Feelings of Power. New York, NY: Routledge.

Bolman, L. \& Deal, T. (2013). Reframing Organizations: Artistry, Choice and Leadership. San Francisco: Jossey-Bass

Bolton, R. (2005). Habermas's theory of communicative action and the theory of social capital. Paper Read at meeting of Western Science Association. San Diego, California.

Branch, W. \& Frankel, R. (2016). Not all stories of professional identity formation are equal: An analysis of formation narratives of highly humanistic physicians. Patient Education Counseling 99. 13941399.

Briggs, (2007). Explore professional identity in leadership in higher education in colleges. School Leadership and Management. (27) 5, 471-485. 
Brozo, W. (2010). Challenges and possibilities of Response to Intervention for adolescent literacy. Journal of Adolescent and Adult Literacy, 53 (4), 277-281.

Buxton, C., Kayumova, S \& Allexsaht-Snider, M. (2013). Teacher, researcher, and accountability discourses: Creating spaces for democratic science teaching practices in middle schools.

Democracy \& Education. 21. (2).

Cabbage, K. (2016). Language and literacy: What SLPs can (and should) bring to the table. Retrieved from May 6. 2016 from http://www.risha.org.

Cabbage, K.; Adams, (1990). Beginning to Read: Thinking and Learning about Principles.

Callard, F. \& Fitzgerald, D. (2014) Rethinking Interdisciplinarity across the social sciences and neurosciences: Experiment entanglements. Theory Culture and Society. London: Palgrave.

Carlisle, J. F., \& Rice, M. S. (2002). Improving reading comprehension: Research-based principles and practices. Baltimore, MD: York Press.

Carspecken, P. F. (1996) Critical Ethnography in Educational Research: A Theoretical and Practical Guide. New York, NY: Routledge.

Carspecken, L. \& Carspecken, P. (2013). Qualitative research: A reader in philosophy, core concepts, and practice. Peter Lang. ISBN: 978-14331-0472-5.

Catts, H. W., Fey, M. E., Tomblin, J. B., Zhang, X. (2002). A longitudinal investigation of reading outcomes in children with language impairments. Journal of Speech and Hearing Research. 45, 11421147.

Clarke, M. (2009). The Ethico-Politics of Teacher Identity. Educational Philosophy and Theory, 41(2), 185-200. Retrieved from http://search.ebscohost.com.uri.idm.oclc.org/login.

Clouder, L. (2005) Caring as a threshold concept: Transforming students in higher education into healthcare professionals. Teaching in Higher Education. 10 (4), 505-517.

Consolini, M., Carson, L. Miller, S., Johnston, R. (2009) Survey of SLPs: RTI Process In Georgia Public Schools. Retrieved on April 12, 2016 fromwww.asha.org. 
Cramer, L. (2015). Inequities of intervention among culturally and Linguistically diverse students. Penn GSE Perspectives on Urban Education, 12(1), 14-21.

Crane, S. J., \& Iwanicki, E. F. (1986). Perceived role conflict, role ambiguity, and burnout among special education teachers. Remedial and Special Education, 7(2), 24-31.

Creswell, J.C. (2014). Research Design: Qualitative, Quantitative, and Mixed Method Approaches. California: Sage Publication.

DeBray, E. H. (2005). Chapter 2: Partisanship and ideology in the ESEA reauthorization in the $106^{\text {th }}$ and 107thCongresses: Foundations for the new political landscape of federal education policy. Review of Research in Education, 29, 29-50. Retrieved fromhttp://rre.aera.net on July 27, 2011.

Denzin, N.K. \& Lincoln, Y.S (1994). Handbook of Qualitative at Research. Thousand Oaks, CA: Sage

Deshler, D., Palincsar, A., Biancarosa, G., \& Nair, M. (2007). Informed choices for struggling adolescent readers. Newark, DE: International Reading Association.

Danielewicz J. (2001). Teaching Selves: Identity, Pedagogy, and Teacher Education Albany, NY: State University of New York Press.

Donovan, M. \& Cross, S. (2002).Minority students in special education and gifted classes. National American Press. Washington D.C

Dublinske, S. (2014). Developing a strategic plan for a program in communication sciences and disorders. ASHA Leader. Retrieved on March 15, 2016 from http://www.asha.org/academic/developing/strategic.

Edgar, D.L. \& Rosa-Hugo, L.I (2007). The critical shortage of speechlanguage pathologists in the public-school setting: Features of the work environment that affect recruitment and retention. Language Speech and Hearing Services in Schools, 38, 31-46.

Edsight. Connecticut State School Education Repository. Retrived from data.ct.gov on February 2, 2018.

Ehren, Lybolt, \& Gottfried, 2010 RTI in Chicago Schools: Lessons learned. Conference Presentation 
Ehren, Barbara \& Deshler, Donald \& Graner, Patricia. (2010). Using the Content Literacy Continuum as a Framework for Implementing RTI in Secondary Schools. Theory into Practice. 49. 315-322. 10.1080/00405841.2010.510760.

Elksnin, L., \& Capilouto, G. (1994). Speech-language pathologists' perceptions of integrated service delivery delivery in school settings. Language, Speech, and Hearing Services in Schools. (25) 258-267.

Eraut, M. (2008). How professionals learn in the workplace. Surrey Centre For Excellence in Professional Training and Excellence (SCEPTrE). Training and Excellence. 1-29.

Espinoza, L. (1994). Multi-identity: Community and Culture. Social, Policy, and Culture. 23

Fallon (2012). New Perspectives in Special Education: Contemporary Philosophy. New York, NY: Routledge.

Fallon, K. A., Katz, L. A. (2011). Providing written language services in the schools: The time is now. Language, Speech, and Hearing Services in Schools, 42, 3-17.

Farquharson (2012). Working memory processes in children with and without speech sound disorders (Unpublished dissertation). University of Nebraska-Lincoln, Lincoln, NE.

Federal Register 2006, 46540 Children Disability Act https://www.govinfo.gov/content/pkg/FR-2014-06-24/pdf/FR2014-06-24.pdf.

Fisher, D., \& Frey, N. (2014). Close reading as an intervention for struggling middle school readers. Journal of Adolescent and Adult Literarcy. (57) 5, 367-376.

Fitzgerald versus Fairfax County School District. Retrieved from https://www.courtlistener.com/opinion/2390122/fitzgerald-vfairfax-county-school-bd/ on February 2, 2018.

Flipsen, P., Jr. (2002). Longitudinal changes in articulation rate and phonetic phrase length in children with speech delay. Journal of Speech, Language, and Hearing Research, 45, 100-110. 
Foss, S \& Waters, E. (2007). Destination Dissertation: A Traveler's Guide to a Done Dissertation. New York: Rowman \& Littlefield Publishers.

Fowler, F. J. (2014). Survey Research Methods ( $5^{\text {th }}$ Ed). Sage Publication Inc.

Fraenkel, J., Wallen, N. \& Hyun, H. (2012). How to Design and Evaluate Research in Education. New York: McGraw Hill.

Frey, L., Botan, C., \& Kreps, G. (1999). Investigating communication: An introduction to research methods. (2nd Ed.) Boston: Allyn \& Bacon.

Fuchs, D., \& Fuchs, L. S. (2006). Introduction to response to intervention: What, why, and how valid is it? Reading Research Quarterly, 41, 93-99.

Fuchs, D. Fuchs, L.S \& Compton, D.L. (2011). RTI Problems \& Possibilities. Sage Publications.

Fuchs, D., Fuchs, L. S., \& Compton, D. L. (2012). Smart RTI: A next -generation approach to multilevel prevention. Exceptional children, 78 (3), 263-279.

Fuchs, L., Fuchs, D. \& Hollenbeck, K. (2007). Extending response to intervention to mathematics at first and third grade. Learning Disabilities Research \& Practice, 22 (1). 13-24.

Fuchs, L. Fuchs, D. \& Stecker, P. (2010). The blurring of special education in a new continuum of general education placements and services. Exceptional Children, 76, 301-323.

Foucault, M. (1979). The subject of power. Critical Inquiry. New York: Vintage Books.

Gee, J. P. (2014). How to Do Discourse Analysis: A Tool Kit $\left({ }^{\text {st }}{ }^{\text {Ed }}\right)$. New York, NY: Routledge.

Gee, J. P. (2001). Identity as an analytic lens for research in education. America Education Review Association. Vol. 25, 99-125. Retrieved on December 11, 2015 from www.jstor.org.

Georgiou, St., Fousiani, K., Michaelides, M., \& Stavrinides, P. (2013). Cultural value orientation and authoritarian parenting as parameters of bullying and victimization at school. International Journal of Psychology. 
Giangreco, M. Prelock, P. \& Turnbull, H (2010). An issue hiding in plain sight: when are speech language pathologists' special educators rather than related service providers? Learning Speech, and Hearing Services in Schools. 41, 531-542.

Gillam, R. B., \& Johnston, J. R.(1992). Spoken and written language relationships in language/learning-impaired and normally achieving school-age children. Journal of Speech and Hearing Research, 35(6), 1303-1315

Gillam, S. L., \& Justice, L. (2010). RTI progress monitoring tools: Assessing primary-grade students in response -to-intervention programs. American Speech-Language-Hearing Leader, 12-15. Retrieved from www.asha.org

Gillam, R. B., \& Ukrainetz, T.A. (2006). Language intervention though literature-based units. In Contextualized Language Intervention: Scaffolding PreK-12 Literacy Achievement. Eau Claire, WI: Thinking Publications, 59-94.

Goldfarb, J. (1991). The cynical society. Chicago. University of Chicago Press.

Gough, P. B., \& Tunmer, W. E. (1986). Decoding, reading, and reading disability. Remedial and Special Education, 7(1), 6-10. https://doi.org/10.1177/074193258600700104

Graham. L. (2007). Speaking of "disorderly" objects: A poetics of pedagogical discourse. Discourse Studies in the Cultural Politics of Education, (28), 1, 1-20.

Guba, E. \& Lincoln, Y. (1998). Paradigmatic controversies, Contradictions, and emerging confluences. Landscape of Qualitative Research. Thousand Oaks, CA. Sage Publishers.

Harlacher, J. \& Nelson, N. \& Sanford, A. (2010). The "I" in RTI research-based Factor in intensifying instruction. Teaching Exceptional Children. 42, 30-38.

Harn, Ogletree, \& Bradshaw \& Ogletree (1999). The speechpathologists in Schools changing roles. Intervention in Schools, 34 (3), 160-169.

Hatt, B. (2007). Street smarts vs. book smarts: The figured worlds of smartness in the lives of marginalized, urban youth. The Urban Review. 
Holden, M., Buck, E., Luk , J., Ambrin, F., Boisaubin, E., Clark, M., Mihalic, A., Sadler, J. (2015). Professional identity formation: creating a longitudinal framework through TIME (Transformation in medical education). The Journal Association of American Medical Colleges.

Holland, D. Lachicotte, W. Skinner, D. and Cain, C (1998). Identity and Agency in Cultural Worlds. Cambridge, MA: Harvard University Press.

Hoover, John. (2008). National implementation of response to intervention (RTI): Research summary of John J. Hoover, Julia S. Sarris. Six Essential Instructional Roles to Implement Response to Intervention Models: Perceptions of Highly Qualified Special Educators. American Journal of Educational Research. 2014; 2(5):257-266. doi: 10.12691/education-2-5-4.

IDEA Partnerships. (IDEA, 1974, 2004[RP2]).Retrieved on November 25, 2013 fromhttp://ideapartnership.org

Izadinia, M (2014). Teacher educators' identity: a review of literature. European Journal of Teacher Education. 37. 10.1080/02619768.2014.947025.

Johnson, C. Beitchman, J. Brownie, E. (2009). Twenty-year follow-up of children with and without speech-language impairments: Family, education, occupational, and quality of life outcomes. Retrieved http://doi.org/10/1044/1058-03660 (2009/08) on March 31, 2019.

Johnson, M. Cowin, L. Wilson, I \& Young, H. (2012) Professional identity and nursing: Contemporary theoretical development International Nursing Review ,59(4), 562-596.

Justice, L. \& Kaderavek, J. (2004). Embedded-Explicit Emergent Literacy Intervention II. Language, Speech, and Hearing Services in Schools, 35, 201-11. 10.1044/0161-1461(2004/020).

Justice, L.M., Logan, J.A., Schmitt, M.B., \& Jiang, H. (2016). Designing effective speech language interventions for children in the public schools leverage the spacing effect. Journal of Speech and Hearing.

Katz, L. Maag, A, Fallon, K.A, Blenkarn, K. \& Smith, M.K (2010). What makes a caseload (un)manageable? school-based speechlanguage pathologists speak. Language Speech Hearing Services Schools, 41 (2),139-151. 
Kaloi, L. (2011). RTI overview. Retrieved on March 3, 2015 from http://www.rtinetwork.org/about-us/contributors/kaloi-laura

Kamhi, A. G., Catts, H. W. (2012). Language and Reading Disabilities (3rd Ed.). New York, NY. Pearson.

Kavale, K.A., Holdnack, J.A., \& Mostert, M.P. (2005). Responsiveness to intervention and the identification of specific learning disability: A critique and alternative proposal. Learning Disability Quarterly, 28, 2-16.

Kidcounts Countbook (2017). Retrieved on March 19, 2019 from http://www.rikidscount.org/Portals/0/Uploads/Documents/Factboo k\%202017/2017\%20RI\%20Kids\%20Count\%20Factbook\%20for\% 20website.pdf

Kleinman, S. \& Copp, M. (1993). Emotions and Fieldwork:Qualitative Research Methods Series 28. London, Sage Publications.

Kozleski, E. B. \& Huber, J. J., (2010). Systemic change for RTI: Embedding change within a critical framework. Theory into Practice, (49), 258-264.

Laing-Gillam, S., Gillam, R., Fargo, J., Olszewski, A., \& Segura, H. (2017). MISL: Progress-Monitoring Instrument for Measuring Discourse Narrative Analysis. (Monitoring Indicators of Scholarly Language [MISL].

Lashley, C., \& Boscardin, M.L. (2003). Special education administration at crossroads. Journal of Special Education Leadership, 16, 63-75.

Lahey, E. (1998). Nonword repetitions of children with specific learning impairments: Explorations of some explanations for their inaccuracies. Applied Psycholinguistics, 19, 279-309.

Liles, B., Duffy, R. Merritt, D. \& Purcell, S.(1995 ). Measurement of narrative discourse in school children with language disorders. Journal of Speech and Hearing, 38 (2), 415-425.

Ladson-Billings, G.\& Tate, W. (2006). Education in the Public Interest. Lipman, (2005). “This is America” 2005: The political economy of education against public interest. Education in the Public Interest. Denver, MA. Teachers College. 
Lave, J. \& Wenger, E. (1991). Situated Learning: Legitimate Peripheral Participation. Cambridge: Cambridge University Press.

Lewis, B. A., Freebairn, L., Tag, J., Ciesla, A. A., Iyengar, S. K., Stein, C. M., \& Taylor, H. G. (2015). Adolescent outcomes of children with early speech sound disorders with and without language impairment. American Journal of Speech-Language Pathology, 24 (2), 150-163. doi:10.1044/2014_AJSLP-14-0075

Lincoln, Y. S., \& Guba, E. G. (1985). Naturalistic Inquiry. Newbury Park, CA: Sage Publications.

Mc Eneany, J., Lose, M. \& Schwartz, R. (2000). A transactional perspective on reading and response to intervention. Reading Research Quarterly, (41) 1.

Mc Guinn, P. (2015). Complicated politics to the core. Phi Delta Kappan. Thousand Oaks, CA: Sage Publications, 97, 14-19.

Mc Namara, K., Hindenlang, J \& Cascella, P. (2004) Discharge Practices in Clinical Settings. Contemporary Issues in Communication Science and Disorders, 31, 182-190.

Mc Quat, R.C. (2007). An investigation of agency and marginality in special education. Journal of International Special Education, 3 (3), 37-85.

Machi, L. \& McEvoy, B. (2012). The Literature Review: Six Steps to Success $\left(2^{\text {nd }} E d\right)$. London. Sage Publications.

Martin, C. (2009). R.S. Peters and Jurgen Habermas: Presuppositions of practical reason and educational justice. Educational Theory, 59 (1), 1-15.

Martinez, R. \& Young, A. (2011). Response to Intervention: How is it practiced and perceived? International Journal of Special Education, 26 (1), 44-52.

Marzano, R.J. (2004). Building Background Knowledge for Academic Achievement:Research of What Works in Schools. Alexandria, VA: Association for Supervision and Curriculum Development.

Merriam, S. Caffarella, R. \& Baumgartner, L. (2007). Learning in Adulthood: A Comprehensive Guide. San Francisco, CA. JosseyBass. 
Mezirow, J (1985). A critical theory of self-directed learning. In S. Brookfield (Ed) Self-directed learning "From Theory to Practice" 17-30. New Directions for Continuing Education. 25. San Francisco: Jossey-Bass.

Miles, M.B. \& Huberman, A.M. (1994) Qualitative Data Analysis: An Expanded Sourcebook (2nd. Ed). London: Sage

Nippold, M. (2017). Reading comprehension in adolescents: Understanding underlying language impairments. Language, Speech, and Hearing Services in Schools, 48, 125-131.

Nippold, M. (2006). Later language development. Austin, TX:Pro-Ed

Nippold, M., Ward-Lonergan, J., \& Fanning, J. (2005). Persuasive writing in children, adolescents, and adults: a study of syntactic, semantic, and pragmatic development. Language, Speech, and Hearing Services in Schools, 36, 125-138.

Noddings, N. (2005). The Challenge to Care in Schools; An Alternative Approach to Education. New York, Ny. Teachers College Press.

Nygreen, K. (2013). These Kids: Identity, Agency, and Social Justice. The University of Chicago Press.

Patton, M.Q. (2002). Qualitative Research \& Evaluation Methods (3 ${ }^{\text {rd }}$ $E d$.). Thousand Oaks, CA: Sage Publications.

Paterson, F. R. A. (1998). Mandating methodology: Promoting the use of phonics through state statute. In K. Goodman (Ed.), In defense of good teaching: What teachers need to know about the "reading wars", 107-125. York, ME: Stenhouse.

Paterson, M. Higgs, J. Wilcox, S. \& Villenue, M. (2002). Caring as a 'threshold concept': transforming students in higher education to health(care) professionals. Teaching in Higher Education. (10)4, 505-515.

Paterson, M. Higgs, J, Wilcox, S, Villenue, M. (2012). Self-directed learning in clinical reading in self-directed learning, key dimension in professional education and socialization. Focus on Health Professional Education, (4), 3, 5-21.

Paulson, L., Koester, L., Mell, D. \& Nielson, M, (2009). Advancing Language and Literacy Services for Adolescents Students. ASHA Presentation. 
Pearson, M., Clavenna-Deane, B. \& Supon Carter, K. (2015). Job attitudes of special educators related to inclusion of students with significant disabilities. International Journal of Special Education, 30 (2).

Peter, B., Button, L., Stoel-Gammon, C., Chapman, K., \& Raskind, W. (2013). Deficits in sequential processing manifest in motor and linguistic tasks in a multigenerational family with childhood apraxia of speech. Clinical Linguistics \& Phonetics, 27, 10.3109/02699206.2012.736011.

Popham, W. J., and D. Berliner (March 24-28, 2008). Empirically determining the instructional sensitivity of an accountability test. Paper presented at the annual meeting of the American Educational Research Association, New York

Puma, M., Karweit, N., Price, C., Ricciuti, A., Thompson, W. VadenKiernan, M.(1997). Prospects: Final Report on Student Outcomes. Washington D.C. Department of Education, Office of Planning and Evaluation Services.

Pyle, A. (2011). Considering coherence: Teacher perceptions of the competing agendas of RTI and an existing special education model. Exceptionality Education International, 21, 66-81.

Pyle, N., \& Vaughn, S. (2012). Remediating reading difficulties in a response to intervention model with secondary students. Psychology in the Schools, 49, 273-284.

Quinn, L. (2012). Studies in higher education understanding resistance: An analysis of discourse in academic staff development, Studies in Higher Education. Routledge Taylor \& Francis Group.

Raitano, N. A., Pennington, B. F., Tunick, R. A., Boada, R., Shriberg, L. D. (2004). Pre-literacy skills of subgroups of children with phonological disorder. Journal of Child Psychology and Psychiatry, 45, 821-835.

Rawls, J. (1971). Theory of Justice. Harvard University Press.

Reid, A., Dahlgren, P. Petocz, P., Dahlgren, M. (2008). Identity and Engagement in professional identity formation. Studies in Higher Education. (33), 6, 729-742. 
Regulations of the Rhode Island Board of Regents for

Elementary and Secondary Education Governing the Education of Children with Disabilities. Retrieved on March 1,

2015 from https://sos.ri.gov/documents/archives/regdocs/released /pdf.DESE.

Reynolds, C. \& Shaywitz, S. (2009). Response to intervention: Ready or not? Or from wait-to-watch them fail. School Psychology Quarterly, 24 (2), 130-145.

Richmond, R. \& Kurth, L.A. (1999). Moving from outside to inside: High school students' use of apprenticeships as vehicles for entering the culture and practice of science, the culture and practice of science. Journal of Science Teaching. Retrieved on February 2, 2019 from https://doi.org/10.1002/(SICI)1098-2736(199908)36:6<677::AIDTEA6>3.0.CO;2-\%23

Rinaldi, C., Averill, O. H., Stuart, S. (2011). Response to intervention: Educators' perception of a three-year rti collaborative reform effort in an urban elementary school. Journal of Education. 43-53.

Rogers, R. (2011). An Introduction to Critical Discourse Analysis in Education (2 ${ }^{\text {nd }}$ Ed). New York, NY: Routledge.

Robinson, R. (2005). Readings in Reading Instruction: Its History, Theory, and Development. Boston, MA. Pearson.

Rothenberg, P.S. (2005). White Privilege: Essential Readings on the other side of Racism. New York, NY. Worth Publishers.

Russell, S., \& Kaderavek, J. (1993). Alternative models for collaboration. Language, Speech, and Hearing Services in Schools, 24,76-78.

Ryan, M. \& Bourke, T. (2013). The teacher as reflexive professional: making visible the excluded discourse in teacher standards. Discourse Studies in the Cultural Politics of Education.

RTI Action Network, Tier Interventions. Retrieved on March 3,2017 from http://www.rtinetwork.org/.

Salley, S. (2012). Service delivery models used with adolescents: a pilot study. Perspectives on School-Based Issues. 13. 97. 10.1044/sbi13.4.97. 
Sanger, Friedli, C., Brunken, C. Snow, P., Ritzman, M. (2012). Educators' year-long reactions to the implementation of a response to intervention (RTI) model. JJournal of Ethnographic \& Qualitative Research, (7), 98-107.

Sanisosti, F. Goss, S. \& Noltemeyer, A. (2011). Perspective of special educators' directors on RTI in secondary schools. Contemporary School Psychology, 11, 9-21.

Schoenbach, R. Greenleaf, C; Cziko, C; Hurwitz, L. (1999). Reading for Understanding. Jossey-Bass.

Scull, J. \& Winkler, A.M. (2011). Shifting trends in special education. Fordham Institute Advancing Educational Excellence. Retrieved on November 22, 2014 from http://edexcellencemedia

Shapiro, E. (2019). Tiered instruction and intervention in a response-tointervention model. Retrieved from RTI Network.org on January 3, 2019.

Shinn, M. R. (1989). Curriculum-based measurement: Assessing Special Children. New York: Guilford.

Skebo, C., Lewis, B., Freebaim, L., Tag, J., Ciesla, A., \& Stein, C. (2013). Students with speech sound disorders at three stages of literacy development, Language, Speech, and Hearing Services in Schools. Retrieved on April 26, 2016 from www.ncbi.nlm.gov/pmc/articles/.

Souto -Manning, M. (2014). Critical narrative analysis: the interplay of discourse analysis with narrative analysis. International Journal of Qualitative Studies. Routledge Taylor Group, 27, 159-180.

Snowling, M., Bishop, D. V. M., Stothard, S. E. (2000). Is preschool language impairment at risk factor for dyslexia in adolescence? Journal of Child Psychology and Psychiatry, 41, 587-600.

Swanson, E., Solis, M., Ciullo, S., \& McKenna, J. W. (2012). Special Education Teachers' Perceptions and Instructional Practices in Response to Intervention Implementation. Learning Disability Quarterly, 35(2), 115-126. https://doi.org/10.1177/0731948711432510

Szydlowski, S (2019). Ignorance of the law. Providence Sunday Journal dated June 2, 2019, (46), 1-2. 
Tovani, C. (2003). I Read It, but I Don't Get It. New York: Stenhouse Publishers.

Trede, F \& Loftus, S. (2010). Hermeneutic research: Exploring human understanding. In Researching practice: A discourse on qualitative methodologies. 185-195.

Ukrainetz, T.A. (2006). Teaching narrative structure: coherence, cohesion, and captivation. In Ukrainetz, T.A. (Ed.), Contextualized Language Intervention: Scaffolding PreK-12 Literacy Achievement. Eau Claire, WI: Thinking Publications. 195-246.

Ukrainetz, T., \& Fresquez, E. (2003). What isn't language? A qualitative study of the role of the school speech-language pathologist. Language, Speech, and Hearing Services in Schools, 34, 284298.

Ullman, C. (2012). 'Before I didn't understand anything about white people, but now, I speak English negotiating globally mediated discourses of race, language, and nation. Discourse Studies in the Cultural Politics of Education, 33 (2), 251-266.

Viteritti, J.P (2013). The Federal Role in School Reform: Obama's Race to the Top. Notre Dame Legal Review 2087

Welton, M. R (1995). The critical turn in adult education theory. In M.R. Welton (Ed.) In defense of lifeworld.11-38. Albany State University of New York Press.

Werts, G.W., Lambert, M., \& Carpenter, E. (2009). What special education directors say about RTI. Learning Disability Quarterly, (32), 245-254.

White, R. B., Polly, D., \& Audette, R. H. (2012). A case analysis of an elementary school's implementation of Response to Intervention. Journal of Research in Childhood Education, 26(1), 73-90. doi:10.1080/02568543.2011.63206.

Wisniewski, L. \& Gargiulo, R.M. (1997). Occupational stress and burnout among special educators. Journal of Special Education, (31), 325346.

Wilcox, K. A. Murakami-Ramalho, E., \& Urick, A. (2013). Just-in time pedagogy: Teacher's perspectives on the response to intervention framework. Journal of Research in Childhood Education, 26 (1), 73-90. 
Wilson I, Cowin L.S., Johnson M \& Young H. (2013). Professional identity in medical students: Pedagogical challenges to medical education. Teach Learn Medicine. 369-373.

Wolter, J.A., \& Pike, K. (2015). Dynamic assessment of morphological awareness in third grade children. Language Speech and Hearing Services in the Schools, 46, 112- 126.

Zembylas, M. (2014). "When saying you care is not caring": emotions of disgust, whiteness of ideology." Critical Studies in Education. 55 (3), 319-337.

Zirkel, P (2011) In special education law. Principal Leadership. 